\title{
ANALYSIS OF FACTORS THAT AFFECT PSEUDOTYPING IN RETROVIRUSES
}

\author{
A Dissertation \\ presented to \\ the Faculty of the Graduate School \\ at the University of Missouri-Columbia \\ In Partial Fulfillment \\ of the Requirements for the Degree \\ Doctor of Philosophy \\ by \\ YUL EUM SONG \\ Dr. Marc Johnson, Dissertation Supervisor \\ MAY 2019
}


The undersigned, appointed by the dean of the Graduate School, have examined the dissertation entitled

\title{
ANALYSIS OF FACTORS THAT AFFECT PSEUDOTYPING IN RETROVIRUSES
}

\author{
Presented by Yul Eum Song, \\ A candidate for the degree of doctor of philosophy, \\ and hereby certify that, in their opinion, it is worthy of acceptance.
}

Dr. Marc Johnson

Dr. Donald Burke

Dr. Mark Daniels

Dr. David Pintel

Dr. Bumsuk Hahm 


\section{Dedication}

Heal the world. Make it a better place, for you and for me and the entire human race. -Michael Jackson, from the lyrics of "Heal the World"

"Even the smallest person can change the course of the future." -Galadriel, the movie "The Lord of the Rings"

Then you will know the truth, and the truth will set you free.

-John 8:32, Holy Bible 


\section{ACKNOWLEDGEMENTS}

I thank my advisor Dr. Marc Johnson for his generosity, care, and invaluable help and patience in his mentoring. I learned how to do science, how to love science, and how to think critical under his guidance. I have grown up to become a scientist that I am now today, and without his guidance, this day would never come. He has been a great mentor to me and a scientist I will seek to emulate as a role model in my life.

I thank previous and current lab members for sharing research and having a pleasant lab environment, where we spend most of the time. I thank Terri Lyddon for her warmth and friendship, and her efforts to make the lab work. I thank Dr. Maggie Lange, Dr. Devon Gregory, and Dr. Sanath Kumar Janaka for having great scientific discussions and feedback. I thank Clifton Ricana and Carolyn Robinson for their respect.

I thank my department for the support and help. I thank my committee, Dr. Donald Burke, Dr. Mark Daniels, Dr. Bumsuk Hahm, and Dr. David Pintel for their support and feedback. I especially thank Dr. Bumsuk Hahm, Dr. Madhuvanthi Vijayan, Dr. Prabuddha Sengupta, and Dr. Jennifer Lippincott-Schwartz for giving me great opportunities to participate in their collaborative research projects. I thank Dr. Tiffany Lucas and Daniel Cyburt for our collaborative works. I also thank Dr. Charles Russell for offering me a great job opportunity as a postdoc in his lab.

Finally, I thank my beautiful sister Dr. Han Byul Song and her husband Dr. Samuel Park, and my parents, Dr. Mi Hyung Kim and Kyosuck Song, for their love and support. Without them, I would not dare to begin and move through this challenging journey of Ph.D. I thank my church mentor Dr. Suhwon Lee for her devotion, support, and guidance. I also thank my good friends for their countless support and care. Last, but not least, I thank God for giving me the dream, interest, and patience to pursue research. 


\section{TABLE OF CONTENTS}

Acknowledgements

List of Figures $\quad v$

Abstract vii

I. Introduction

1.1 Retroviruses

1.2 Retroviral genome and proteins

1.3 Retroviral life cycle $\quad 21$

1.4 Pseudotyping compatibility in retroviruses $\quad 32$

1.4.1 Role of retroviral Env cytoplasmic tail _............................................. 32

1.4.2 Role of cellular factors

1.4.3 HIV-1 accessory protein Vpu and its mode of action on GaLV Env ......... 34

II. $\beta \operatorname{TrCP}$ is Required for HIV-1 Vpu Modulation of CD4, GaLV Env, and BST2/Tetherin

2.1 Abstract $\quad 38$

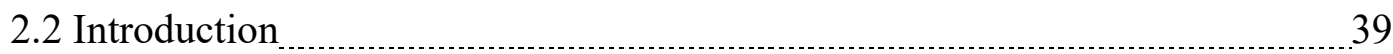

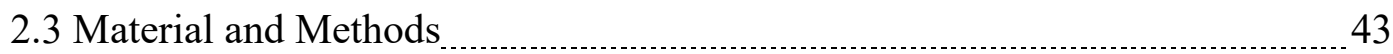

2.4 Results $\ldots$

2.5 Discussion 57

III. Sequence determinants in gammaretroviral Env cytoplasmic tail dictating virusspecific pseudotyping compatibility 
3.1 Abstract

3.3 Introduction 75

3.4 Materials and Methods 76

3.5 Results 82

3.6 Discussion 90

IV. Cell type specific factors dramatically affect functionality of retroviral pseudotyped particles

4.1 Abstract 114

4.2 Introduction 115

4.3 Materials and Methods 117

4.4 Results

4.5 Discussion 128

V. Summary and Discussion 147

VI. Future Directions 150

Literature Cited 151

Vita 175 


\section{List of Figures}

Figure 1.1

Figure $1.2 \ldots \ldots \ldots$

Figure $1.3 \quad 11$

Figure $1.4 \ldots \ldots \ldots+\cdots$

Figure $1.5 \quad 15$

Figure 1.6

Figure 1.7

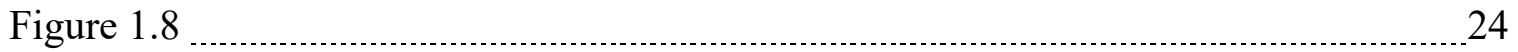

Figure 1.9

Figure $1.10 \quad 28$

Figure $1.11 \ldots \ldots \ldots$

Figure $1.12 \ldots 36$

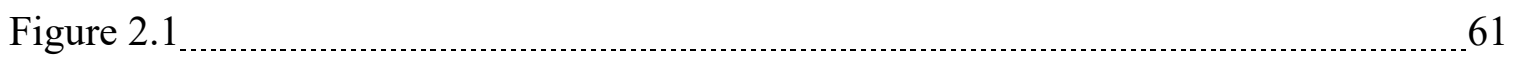

Figure $2.2 \quad 63$

Figure $2.3 \ldots \ldots$

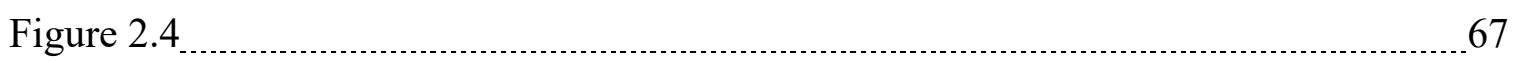

Figure $2.5 \quad 69$

Figure 2.6

Figure $3.1 \quad 96$

Figure $3.2+\ldots$

Figure $3.3 \quad 100$

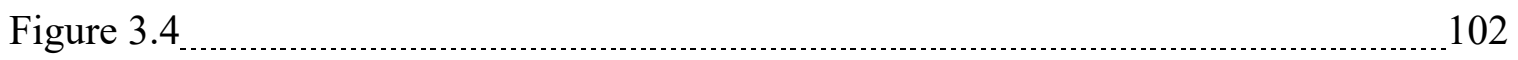

Figure $3.5 \ldots \ldots$

Figure $3.6 \quad 106$ 
Figure $3.7 \quad 108$

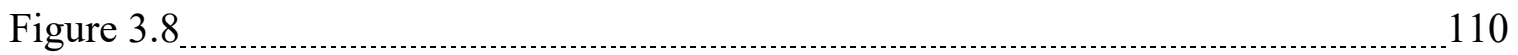

Figure $3.9 \quad 112$

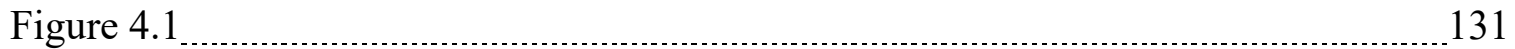

Figure 4.2

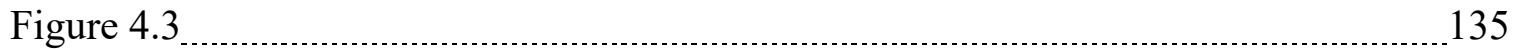

Figure 4.4 137

Figure 4.5

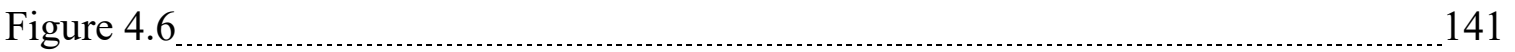

Figure 4.7

Figure $4.8 \ldots \ldots \ldots$ 


\begin{abstract}
$\underline{\text { Abstract }}$
Retroviral vectors can efficiently pseudotype with various envelope glycoproteins; however, the molecular mechanism of glycoprotein acquisition process is not completely understood even with their native Env proteins. Understanding this mechanism would aid development of a safe and efficient retroviral gene therapy vector and in finding an antiviral therapeutic target.

Various factors were reported to affect the glycoprotein acquisition process. Protein-protein interactions and protein-lipid interactions were reported between viral proteins or cellular factors. In the studies outlined here, we examined three factors that affect retroviral pseudotyping: the HIV-1 accessory protein Vpu, the Env cytoplasmic tail, and cell line-specific host factors.

Vpu has been identified to negatively affect Env protein from gibbon ape leukemia virus $(\mathrm{GaLV})$ in a similar way that it down-modulates native targets, CD4 and BST2/tetherin. We take advantage of a novel CRISPR-Cas9 technique to present here that BTrCP is a necessary host factor for Vpu to target GaLV Env, CD4, and BST-2/tetherin.

The role and structure of Env cytoplasmic tails are largely unknown among retroviral proteins. We present that specific sequences in a gammaretroviral cytoplasmic tail affect fusogenicity in a virus-specific way. It suggests that the lipid environment differs between viruses and that these differences affect fusion of Env.

Finally, we present that the cell line specific assembly defect of murine leukemia virus (MLV) particles in HeLa cells. The budding defect of MLV from HeLa cells is restored by fusion with 293FT cells, suggesting that an unknown necessary host factor is deficient in HeLa cells.
\end{abstract}




\section{$\underline{\text { I. Introduction }}$}

\subsection{Retroviruses}

Retroviruses belong to the Retroviridae family, which includes various genera of viruses which infect multiple species of vertebrates. The most pronounced features of retroviruses are reverse-transcription of the viral RNA genome into DNA, and integration of this DNA into the host cell's chromosome. Both steps are critical for the life cycle and pathogenesis of the retrovirus. Some retroviruses are also known for causing cancers by expressing viral oncogenes or disrupting regulatory gene expression as a consequence of integration. The most well-known retrovirus is Human Immunodeficiency Virus-1 (HIV1), which infects humans and causes acquired immune deficiency syndrome (AIDS).

Retroviruses are enveloped viruses of about 100-200nm in diameter that package two copies of their single-stranded (positive strand) RNA genome and other proteins (Figure 1.1) (1,2). Each viral genomic RNA is 5' capped, contains a poly-A tail at the 3' end, and encodes 3 essential genes plus additional accessory genes. Phylogenetic trees based on essential genes show 7 different genera of retroviruses, and this grouping also generally follows the classic classification by the shape of the virus, genome, encoded genes, and the host species (Figure 1.2) $(3,4)$. Based on the coding regions, retroviruses are classified by 'simple' (alpha-, beta-, and gamma-, epsilon-retroviruses) if RNA transcripts are not spliced to produce genes other than Gag, Pol, and Env, or as 'complex' (delta-, spuma-retroviruses, and lentiviruses) if viral RNA splicing generates additional accessory proteins. Some retroviruses (mostly simple retroviruses) have been identified 
that encode additional viral oncogenes such as $v$-src, which is encoded in Rous Sarcoma Virus (RSV).

Most accessory proteins are not required for viral replication but enhance the pathogenesis by supporting viral replication and infection, such as by counteracting host restriction factors. More recently, some simple retroviruses originally classified as 'simple' have been reported to express additional proteins. For example, Mouse Mammary Tumor Virus (MMTV) Rem and Jaagsiekte Sheep Retrovirus (JSRV) Rej protein are translated from an alternative spliced transcript, and both proteins act as a nuclear export trans-acting factor $(5,6)$. Also, Murine Leukemia Virus (MLV) occasionally initiates translation upstream of the Gag gene using a non-ATG start codon to generate GlycoGag, which acts like HIV-1 accessory protein Nef (7). Complex retroviruses encode more accessory genes in addition to the necessary genes. HIV-1, for instance, encodes a total of 6 accessory genes. 
Figure 1.1: Simplified schematic of a retroviral particle.

MA: matrix. CA: capsid. NC: nucleocapsid. PR: protease. RT: reverse transcriptase. IN: integrase. SU: surface domain of Env. TM: transmembrane domain of Env. Image obtained from (1) with permission. 
Figure 1.1: Simplified schematic of a retroviral particle.

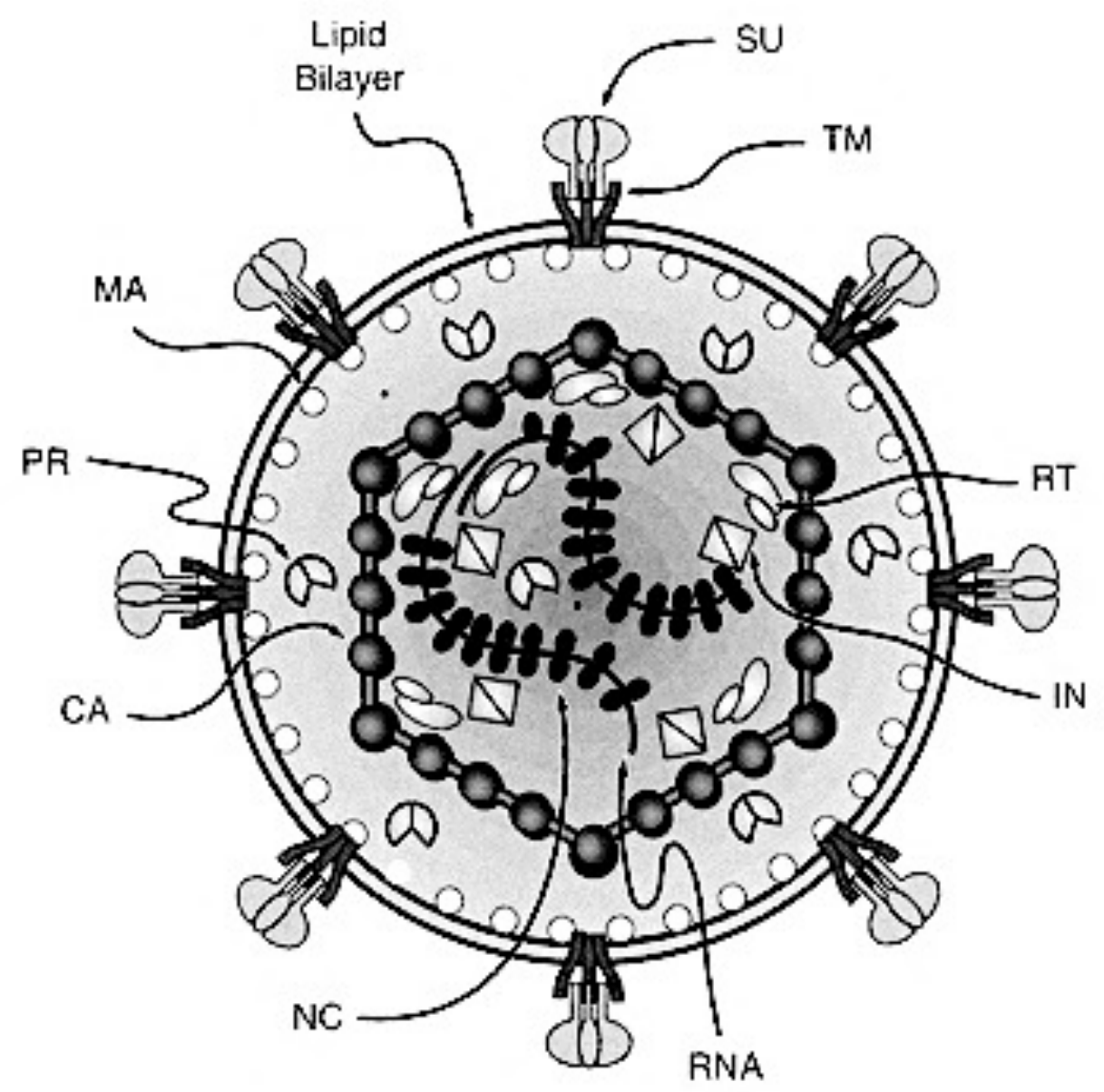




\section{Figure 1.2: Genetic organization of retroviruses.}

(A) Phylogeny of retroviruses. Image obtained from (8) with Creative Commons Attribution License. (B) Genetic organization of prototypic retroviruses. Representative virus from each genera is shown. Image modified from (4) with permission. 
Figure 1.2: Genetic organization of retroviruses.

(A)

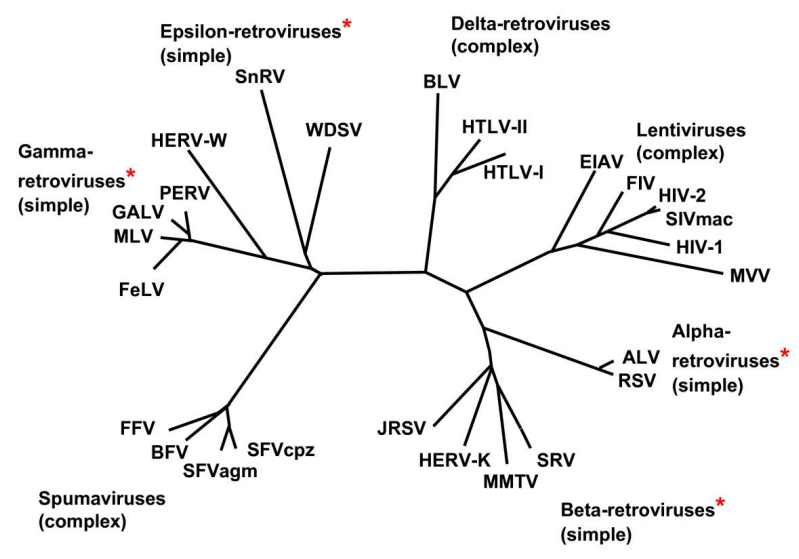

(B)

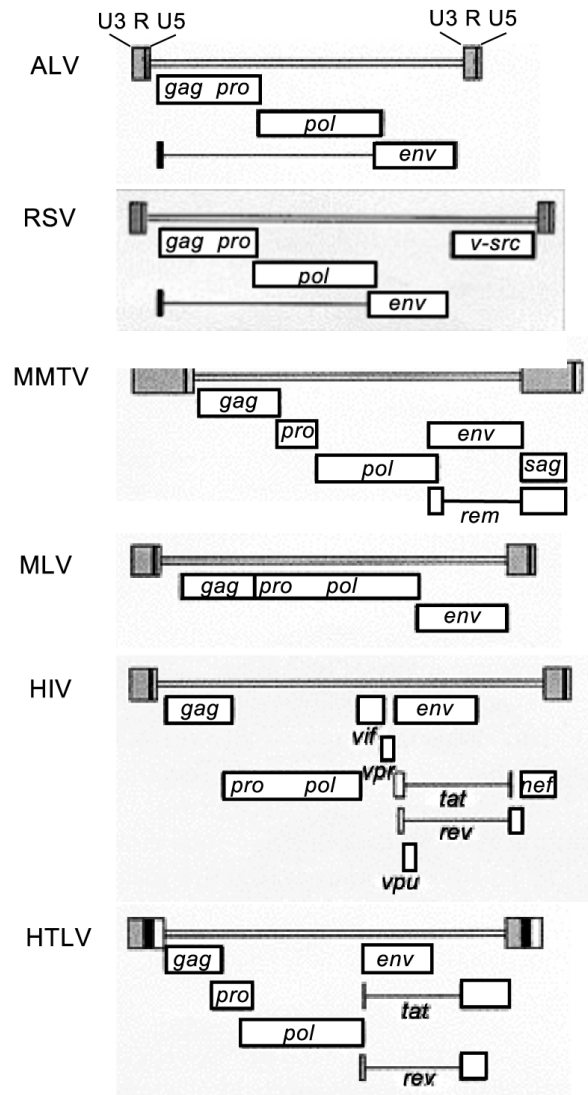

HFV

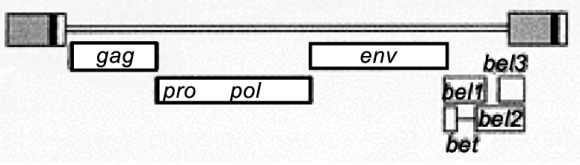

WDSV

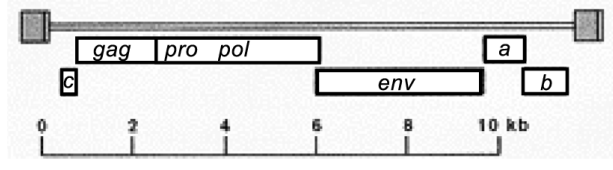




\subsection{Retroviral Genome and Proteins}

The retroviral genome is a dimer of single stranded positive sense RNA, that ranges from 7 to $13 \mathrm{~kb}$ in length. Integrated viral DNA transcribes an unspliced full-length viral RNA that serves both as the packaged genomic RNA as well as the template for the Gag and GagPol proteins. Splicing of the host genome generates several different transcripts that produce Env and other viral proteins (Figure 1.3) (9). The 5'and 3' ends of viral DNA or RNA is called the LTR (long terminal repeat) and contains promoters and RNA elements with various functions (Figure 1.4) (10). Genomic viral RNA has 1 copy of U3 and U5 and several cis-acting elements. Necessary RNA elements include dimerization signal (DIS) for dimerization of genomic RNA, primer binding site (PBS) for the interaction of reverse transcription, and packaging element $(\Psi)$ for packaging genome into viral particle through binding to Nucleocapsid (NC) domain of Gag. Secondary structure of the HIV-1 RNA 5' leader was shown to promote either a monomeric or dimeric conformation of RNA (Figure 1.4B) (11).

Gag is the major retroviral structural protein. Most retroviral Gag proteins have at least 3 domains, Matrix (MA), Capsid (CA), and NC (Figure 1.5). The MA domain from most retroviruses is myristoylated at the second residue (glycine) from the N-terminus, which signals trafficking of Gag to the plasma membrane. MA of RSV, ASLV, and EIAV Gag were exceptions; the myristoylation is not required in these MA domains. In addition, basic residues in MA also contribute to the interaction with acidic phospholipids.

The CA domain forms the major structural core of particles. During maturation (cleavage and separation of Gag domains by activated viral protease), cleaved CA forms an inner shell which surrounds and protects the viral genome. The morphology of mature capsid core is different in various retroviruses (Figure 1.6) (2). Generally, lentiviruses 
exhibit cone-shaped capsid cores while the others exhibit polyhedral or spherical cores. The capsid protein has been shown to be involved in various steps in HIV-1 life cycle including reverse transcription, nuclear import of genome, and integration (12).

The major role of the NC domain is to bind viral genomic RNA through zinc finger motifs and package the genome into viral particles. During infection, $\mathrm{NC}$ also regulates rearrangement of nucleic acid and enhances the reverse transcription process (13).

In addition to the three major domains of Gag, most retroviruses also possess additional polypeptide segments between domains. These include short 'spacer' peptide (SP) or larger domains, at least one of which always contains a 'late domain' motif. Late domains are amino acid motifs that recruit components of cellular ESCRT (endosomal sorting complexes required for transport) machinery that are necessary for virus budding (14). Most retroviruses have this additional domain between MA and CA, while lentiviruses have it downstream of NC.

The Pol protein encodes viral enzymes reverse transcriptase (RT) and integrase (IN). The third viral enzyme protease (PR) is separately encoded either at the c-terminal of Gag (RSV, ASLV), n-terminal of Pol (lentiviruses), or as a separate ORF (MMTV, MPMV, HTLV-1) (Figure 1.2B). During translation, retroviruses efficiently translate Gag and use frameshift $(f s)$ or readthrough (is) mechanisms to occasionally by-pass the stop codon of Gag to produce GagPol (Figure 1.5). About one-tenth to one-twentieth of time, GagPol is translated from the same transcript of Gag with these mechanisms.

RT performs reverse transcription, which converts the RNA genome to DNA; the most pronounced feature of retroviruses. The RT protein of HIV-1 acts as a dimer composed of a long (p66) and a cleaved (p51) from of RT. The C-terminal component of RT protein is the RNAse-H domain, which is cleaved in $\mathrm{p} 51$. IN is responsible for inserting 
viral DNA into the chromosomes of host cells. PR is activated in viral particles during maturation, and cleaves necessary proteins to mature forms, which is required for viruses to be infectious.

Env, the final essential viral protein is a transmembrane glycoprotein, which is critical for infection (Figure 1.7). Env is a class I viral fusion protein and is a major target for the immune system due to the exposure of Env on the surface of viral particles. Env is responsible for interacting with the host cell receptor during infection. In all cases, Env is translated from separate spliced transcript with a signal peptide which leads to initiation of translation in the ER. The precursor Env protein is trafficked to Golgi and cleaved into 2 domains - SU and TM- by a cellular protease. The SU domain is highly glycosylated and responsible for receptor binding. TM domain has transmembrane domain and fusion peptide, which initiates fusion during entry. Both domains are either covalently (Alpha-, Gamma-, Delta-retroviruses) or non-covalently bound (Beta-retroviruses, lentiviruses) (15). Because of the transmembrane domain in TM, the Env protein is anchored on the membrane. Binding of SU to its host receptor protein leads to a conformational change in the Env protein, which is required for fusion of the viral and cellular membranes.

Complex retroviruses encode accessory genes (Figure 1.2). HIV-1 encodes a total of six accessory proteins, Vif, Vpr, Vpu, Tat, Rev, and Nef. Tat binds to the TAR (Transactivation response region) element in newly generated RNA and recruits host factors to enhance transcription of the viral RNA. Rev enhances the nuclear export of unspliced viral RNA by binding an RNA element in the genome called the RRE (Revresponsive element). Vpu downregulates a restriction factor tetherin and enhance virus budding (16). Nef has recently been found to overcome the cellular restriction factor, Serinc5 $(17,18)$. Vif is packaged in viral particle and degrades a restriction factor called 
APOBEC3G, an RNA editing enzyme (19). Vpr is also packaged in viral particles, and has been reported to possess several functions, though the role of Vpr in viral replication is poorly understood. Though simple retroviruses have been believed to not contain accessory proteins, some simple retroviruses have been found to produce atypical 'accessory proteins.' Such examples include gamma-retrovirus GlycoGag and MMTV Rem proteins $(5,20)$. 
Figure 1.3: Examples of alternative splicing patterns in retroviruses.

Simple retroviruses such as MLV and RSV generate each transcript with single splicing event while complex retroviruses such as HIV-1 generate transcripts with multiple alternative splicing events. Image obtained from (9) with permission. 
Figure 1.3: Examples of alternative splicing patterns in retroviruses.
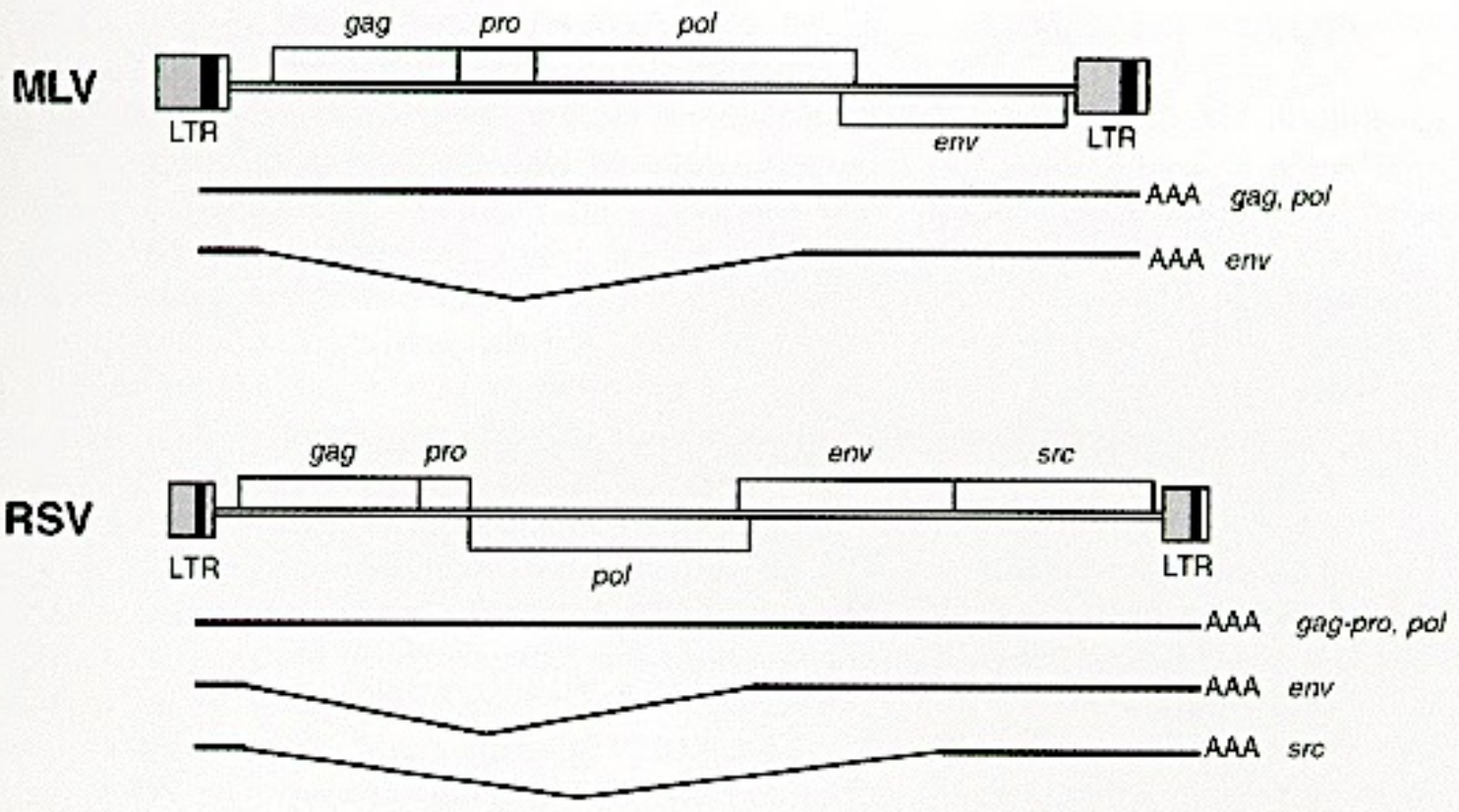

HIV-1
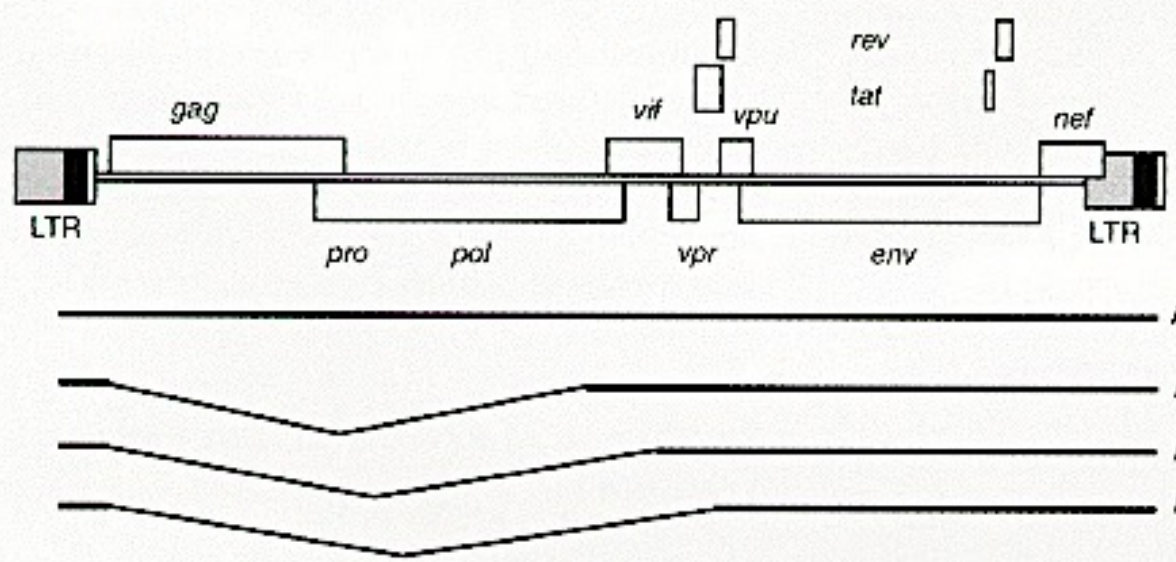

AAA gag pol

AAA VI

AAA wpr

AAA YPU, enV

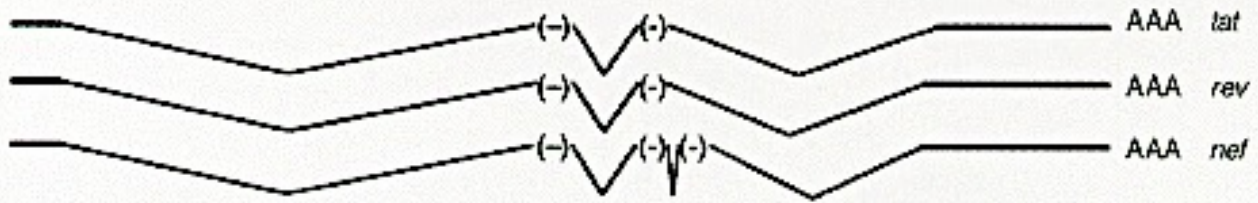




\section{Figure 1.4: Cis-acting elements in retroviral RNA.}

Schematic of secondary structures and cis-acting elements in the 5' LTR of HIV-1 genome. Secondary structure of HIV-1 5' LTR region promotes monomeric (A) or dimeric (B) conformation. Structures are probed with Nuclear Magnetic Resonance (NMR) Spectroscopy (colored regions). Yellow boxes indicate that the structure was probed with truncation or mutation of 5'-leader constructs. Image obtained from (11) with Creative Commons Attribution License. 
Figure 1.4: Cis-acting elements in retroviral RNA.

(a)

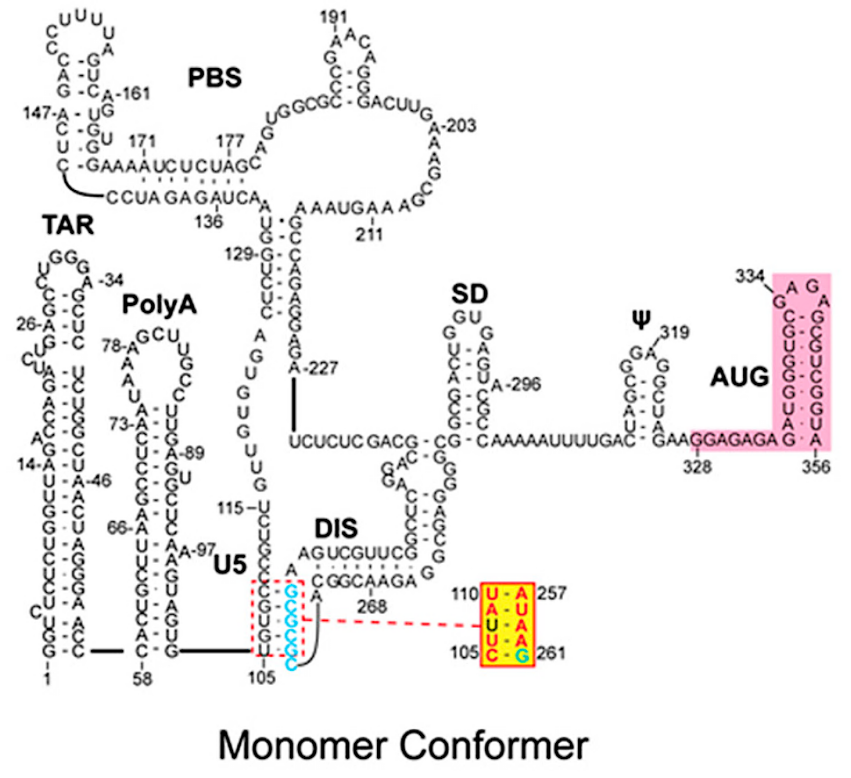

(b)

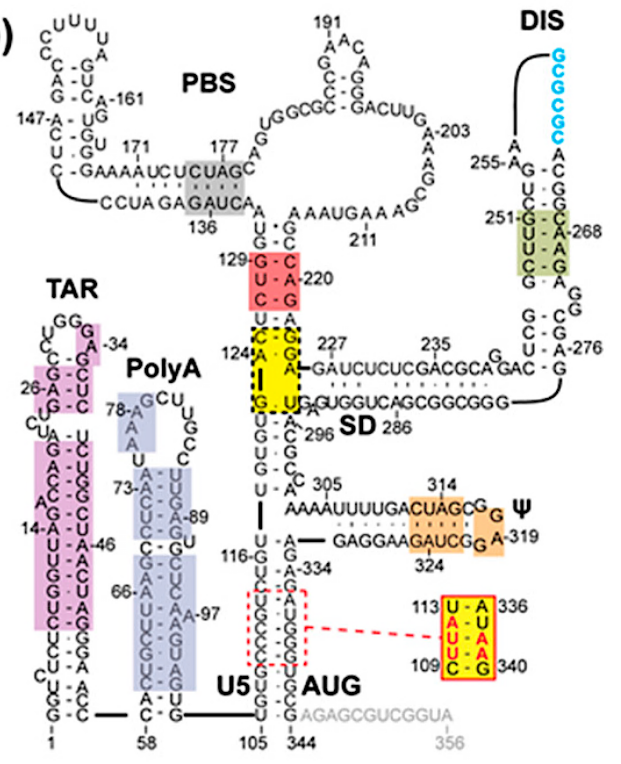

Dimer-Promoting Conformer 
Figure 1.5: Schematic of HIV-1 and MLV GagPol proteins and their translational mechanisms.

Gag and GagPol proteins are translated from the same transcript. HIV-1 GagPol is translated from frameshift $(f s)$, and MLV GagPol is translated from stop codon suppression (is: readthrough). MLV Glyco-Gag (Pr80) is translated from an upstream CUG codon. 
Figure 1.5: Schematic of HIV-1 and MLV GagPol proteins and their translational mechanisms.

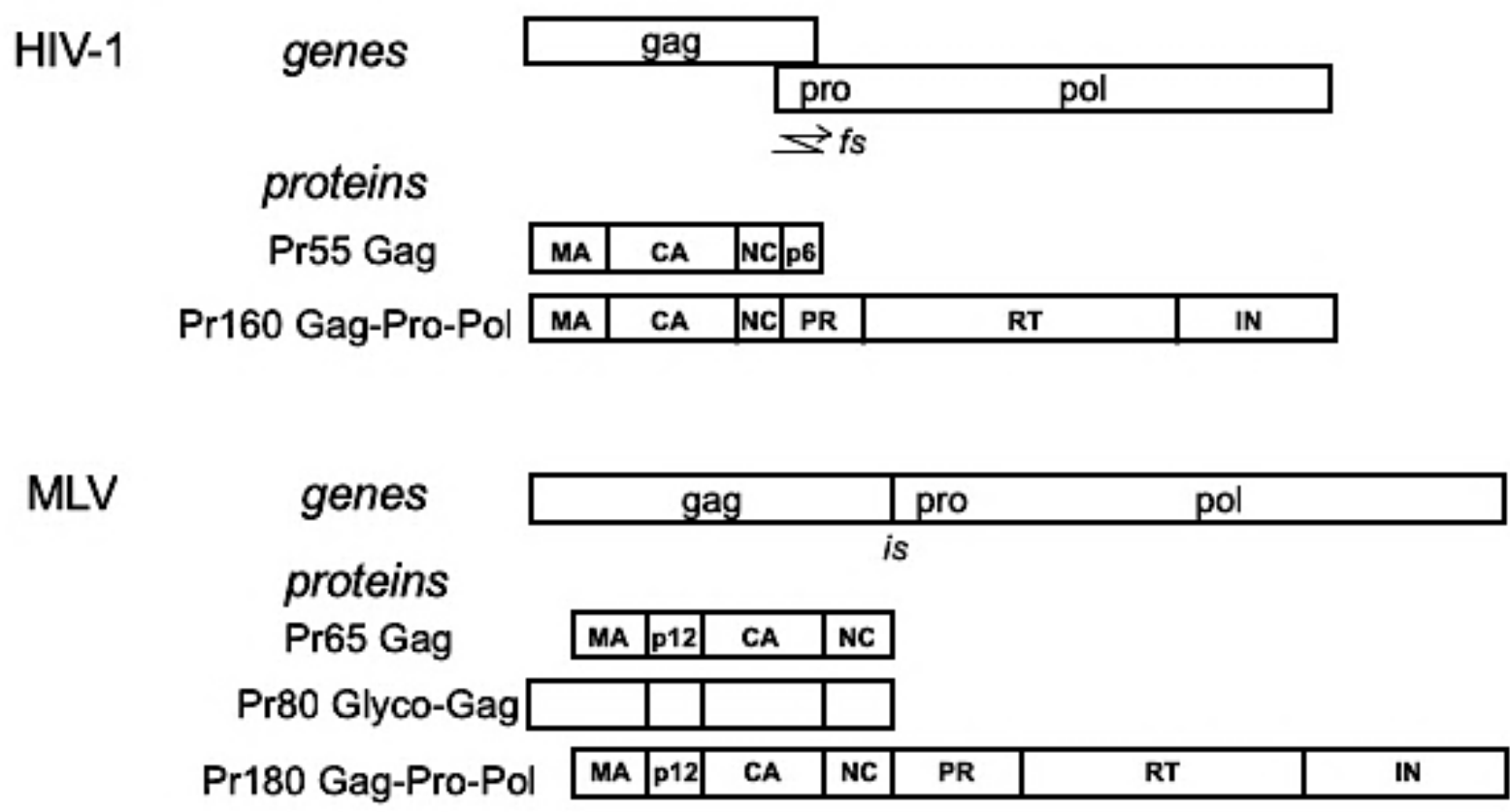


Figure 1.6: Morphology of mature retroviruses by Cryo-EM \& Cryo-ET images.

Shape and diameter of inner core varies between retroviruses. Lentiviruses (HIV-1) have primarily cone-shaped capsid core while the others display polyhedral or nearly spherical. Image obtained from (2) with Creative Commons Attribution License. 
Figure 1.6: Morphology of mature retroviruses by Cryo-EM \& Cryo-ET images.

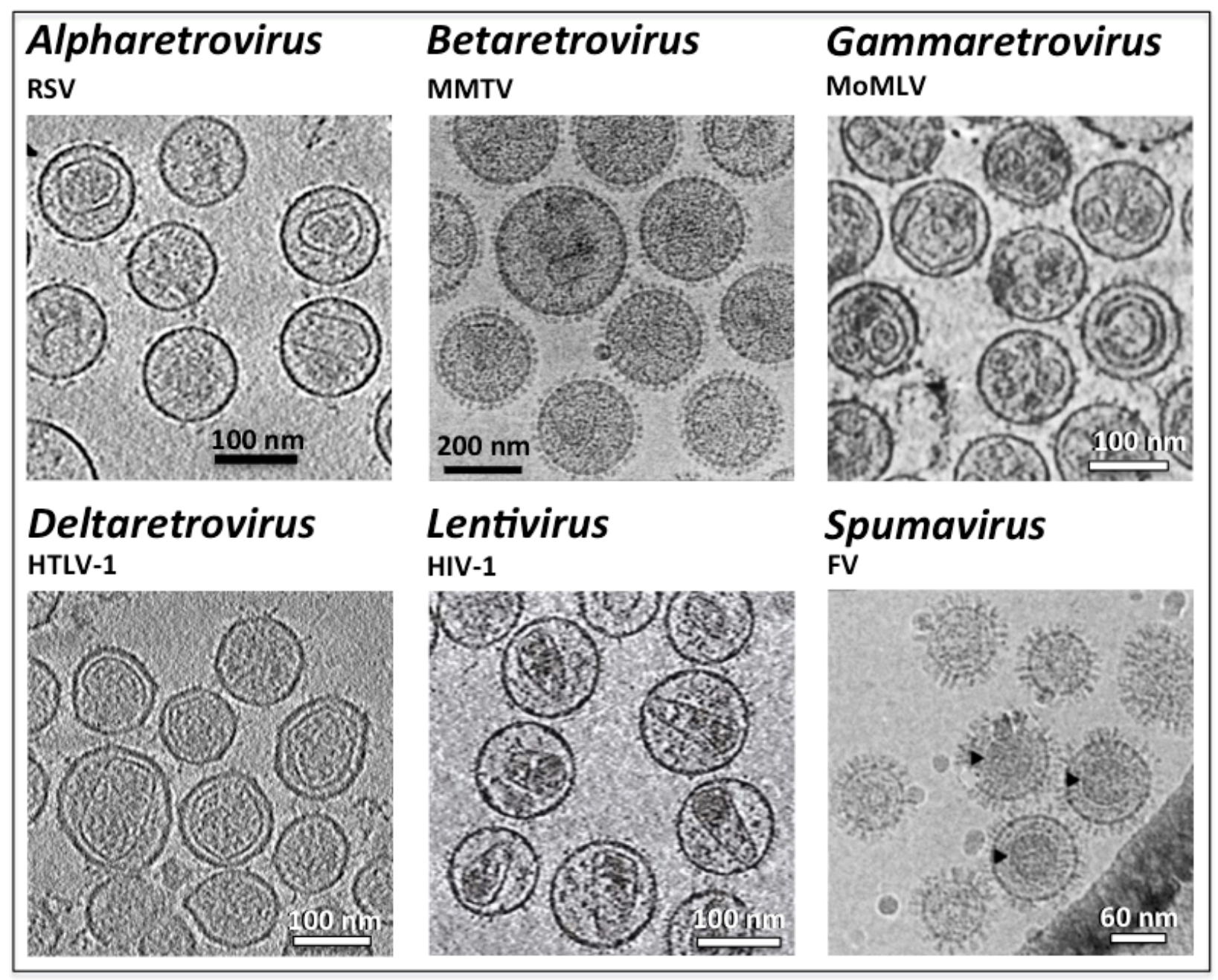


Figure 1.7: Schematic of MLV Env glycoprotein.

Env glycoprotein is translated in ER with the signal peptide (SP), and cleaved to two domains, SU (Surface domain) and TM (Transmembrane domain) by cellular protease. TM consists of N-terminal fusion peptide $(\mathrm{FP})$, helical structure $(\mathrm{NH})$, transmembrane region (TM), and cytoplasmic tail (CT) at the C-terminus. 
Figure 1.7: Schematic of MLV Env glycoprotein.
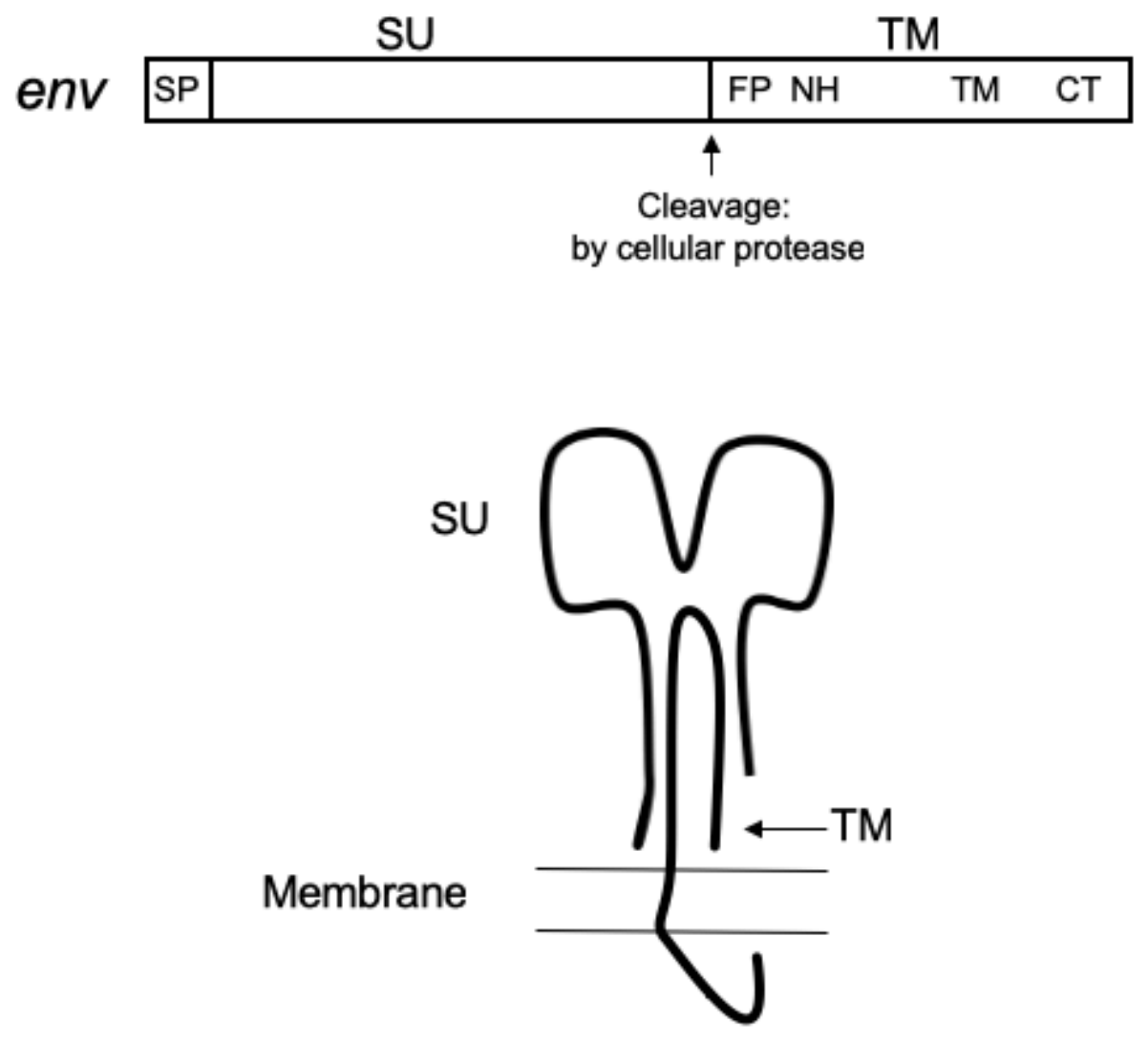


\subsection{Retroviral life cycle}

Retroviruses generally follow a common life cycle (Figure 1.8). In general, the infection is initiated by Env glycoproteins binding to the receptor on target cells. The specificity of Env binding to a receptor determines the host range and the cell tropism. In the case of HIV-1, the CD4 molecule on immune cells (such as T cells or macrophages) is the main receptor, and either CXCR4 or CCR5 is also needed as a coreceptor. This specific interaction leads to the conformational change of Env especially the TM protein, exposing the fusion peptide and initiating the fusion between viral membrane and plasma membrane. After fusion, the inner components of a viral particle enter the cell, and RT reverse transcribes the viral RNA genome into double stranded DNA (Figure 1.9) (21). Briefly, from the positive sense RNA genome, RT initiates synthesis of DNA from tRNA as a primer bound to PBS (primer binding site). As DNA is reverse-transcribed, RNAse H domain of RT degrades RNA strands. When RT reaches the 5' end of RNA LTR, the DNA strand is transferred to the 3' LTR of RNA and annealed to the R (repeated) sequence. DNA synthesis then continues, and the plus stranded DNA is initiated in the other direction from a short sequence of RNA called the PPT (polypurine tract) which is resistant to RNAse H digestion. Finally, the second DNA strand is transferred to the 3' end of the first strand DNA to transcribe double stranded viral DNA.

IN mediates integration of viral DNA into host chromosome (Figure 1.10) (22). First, each 3' end of linear double stranded DNA is cleaved, and the target sequence is attacked and forms a phosphodiester bond at the 3' $\mathrm{OH}$ of viral DNA. This process is called 'strand transfer.' The nick created on the chromosome during strand transfer is repaired by host DNA repair machinery, leaving the viral DNA inserted between duplicated host sequences. 
From integrated viral DNA, initial transcription begins, and viral RNA export to the cytosol is enhanced by trans-acting cellular or viral proteins (23). In the case of HIV-1, Tat enhances transcription by binding the TAR element on newly synthesized viral RNA during the transcription, and Rev increases the nuclear export of unspliced RNA. In cases of simple retroviruses, the constitutive transport element (CTE), a cis-acting element on RNA, recruits host nuclear export proteins such as Tip-associating protein/Nuclear RNA export factor 1 (TAP/NXF1) to export the unspliced RNA. Foamy viruses (FVs), members of Spumaretrovirus family, exhibit a unique RNA export phenotype among retroviruses. FVs don't encode Rev-like accessory proteins and use cis-acting elements to utilize host proteins as the simple retroviruses do. Unlike the other simple retroviruses, FVs recruit HuR and ANP32A/B to utilize the CRM1-dependent export pathway (24).

Viral RNA exported to the cytosol begins the translation of viral proteins from either unspliced RNA or alternatively spliced RNA. GagPol is translated about $5 \%$ of the time through either frameshift or readthrough from the Gag gene. Gag and GagPol multimerize and recruit the viral genome. The myristoylated MA domain of Gag leads the trafficking of Gag to the plasma membrane, which is believed to be the main viral assembly site for HIV-1. The Env protein is recruited to the viral assembly site for incorporation; however, the mechanism by which Env glycoprotein is recruited and incorporated is still not completely understood. Studies suggest the possibility of a common mechanism used by various glycoproteins, but also a specific interaction between Gag and Env $(25,26)$. HIV$1 \mathrm{MA}$ and the TM domain of HIV-1 Env, gp41, were shown to have either direct or indirect interactions that are largely dependent on the cytoplasmic tail of gp41.

Assembled viral particles at the plasma membrane utilize ESCRT machinery of the host cell for viral release (Figure 1.11) (14). The ESCRT pathway is a cellular membrane 
fission process, and three main classes of factor sequentially build up the complex. Adaptor proteins recruit early-acting factors such as Brol domain proteins and ESCRT-I/II complexes, and then these factors recruit late-acting factors such as ESCRT-III subunits. ESCRT-III subunits form filaments for the membrane fission, and the recruited VPS4 ATPase, the final factor, mediates the fission. In most retrovirus, Gag possess more than one late domain in which a sequence motif works as adaptor proteins. $\mathrm{P}(\mathrm{T} / \mathrm{S}) \mathrm{AP}$ was shown to recruit TSG101, a component of ESCRT-1, and YPXL motif was shown to recruit ALIX, Bro1 domain protein. PPXY motif was also reported to be a late domain that recruits the NEDD4 family of E3 ubiquitin ligases. The late domain is not always shown to be required to recruit host factors; HIV-1 Gag does not have a PPXY motif but can recruit NEDD4L through an interaction with a cellular protein AMOT (27). The direct connection between ubiquitination of Gag and ESCRT pathway is still not clear; however, the ubiquitin is essential for viral budding, yet not all Gags are ubiquitinated as in the case of the prototypic foamy virus (PFV) $(28,29)$.

Finally, released retroviral particles are matured by activated PR (viral protease). PR is translated as a part of GagPol through either a frameshift or a readthrough mechanism, or from a separate ORF. Self-activated PR after budding initiates the proteolysis of Gag, Pol, and even Env in certain viruses (mostly gammaretroviruses). The change of viral morphology from an immature viral particle to a mature particle is an apparent difference marked by the rearrangement of cleaved and separated Gag domains. This arrangement also transforms a stable Gag shell to a metastable Gag shell, which is critical for efficient viral entry. Env processing is also required for certain viruses; the c-terminus of gammaretroviral Env was shown to be cleaved by viral protease, which leads to the conformational change of Env to be fusogenically active for infecting new cells (30). 


\section{Figure 1.8: Depiction of HIV-1 life cycle.}

Viral envelope glycoprotein binds to the receptor on the target cell and mediates fusion, through which the inner core of virus enters. RT reverse transcribes to generate double stranded viral DNA, and the pre-integration complex enters the nucleus. Viral DNA integrates into the host cell chromosome, and viral RNA is transcribed to generate genome and viral proteins. Viral proteins are assembled and incorporate genome to form new particles. After budding, PR is activated and maturates the particle, ready for the new infection. RT; Reverse Transcriptase, PR; Protease. Image obtained from NIAID: (https://www.niaid.nih.gov/diseases-conditions/hiv-replication-cycle) 
Figure 1.8: Depiction of HIV-1 life cycle.

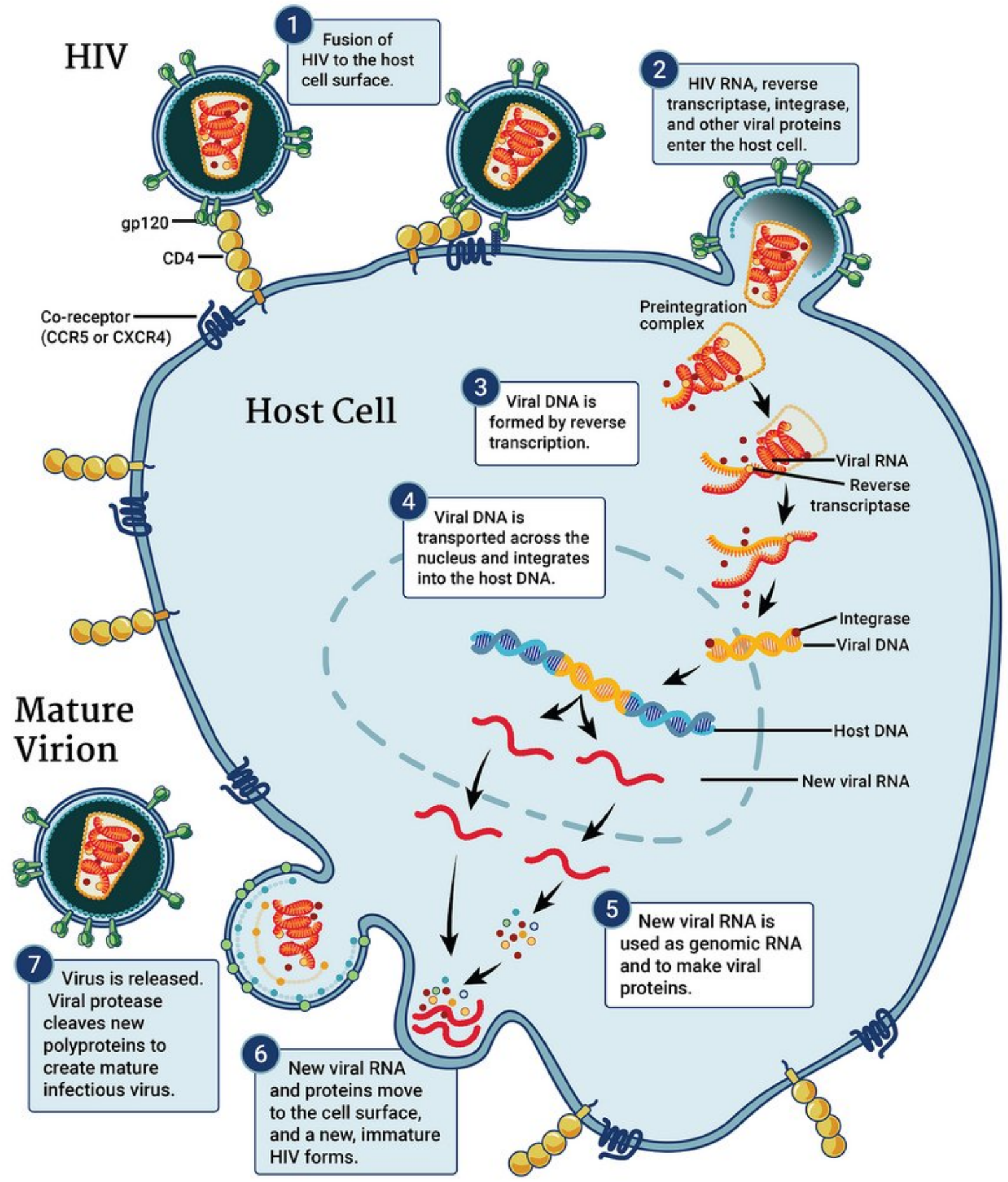




\section{Figure 1.9: Schematic of Reverse transcription.}

RT initiates reverse transcription from the 5'-end of the genome with a cellular tRNA bound to the PBS as a primer. As the viral DNA is generated, RNaseH degrades RNA from RNA-DNA hybrids. The DNA is transferred to the 3'-end, and the reverse transcription continues. The positive strand of viral DNA is synthesized with the PPT region as a primer, leaving double stranded viral DNA. RT; Reverse Transcriptase, PBS; primer binding site, PPT; polypurine tract. Image obtained from (21) with permission. 
Figure 1.9: Schematic of Reverse transcription.

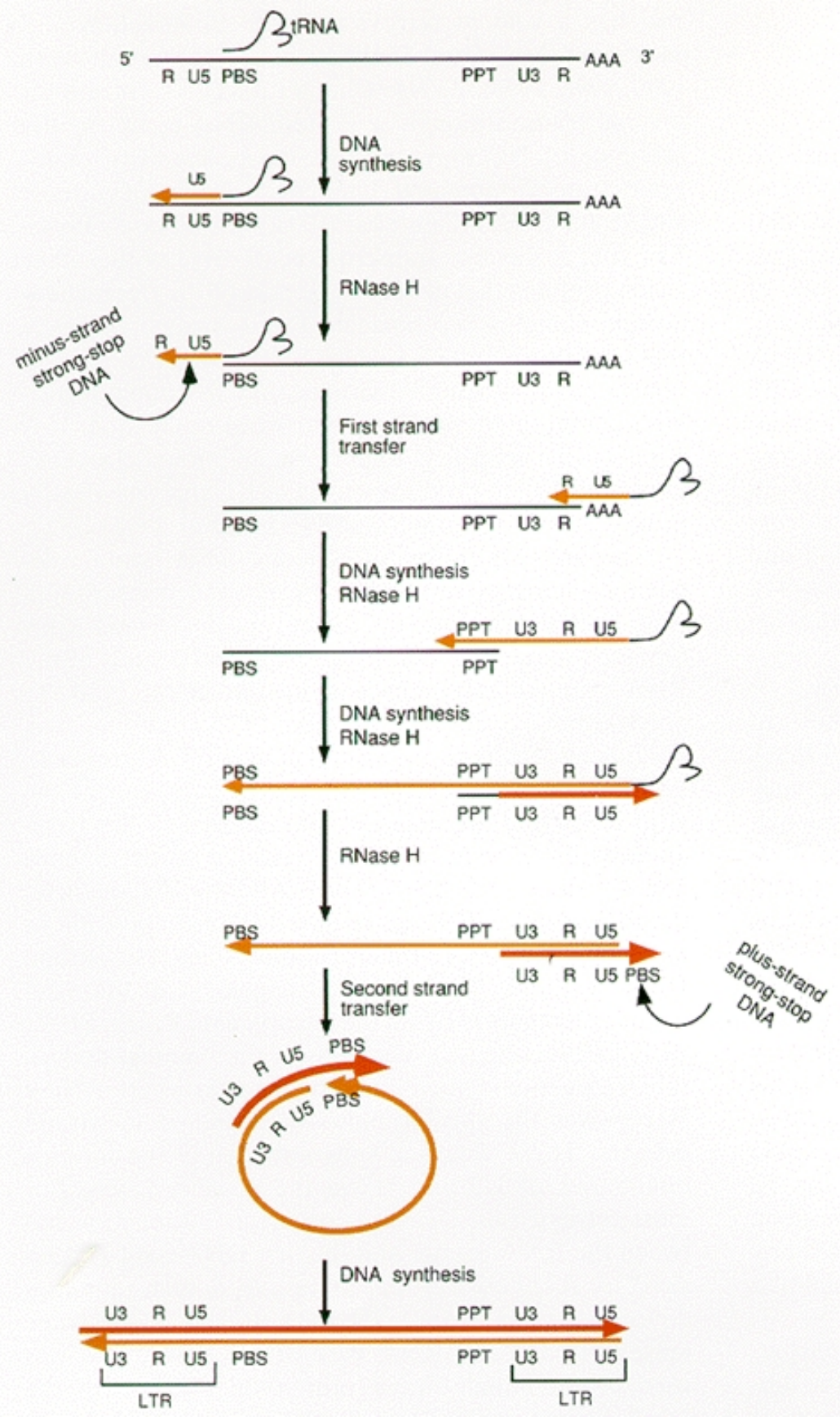


Figure 1.10: Schematic of integration process.

3'-end of double stranded viral DNA is cleaved and attacks the target sequence in chromosome. 3'-OH of each end is annealed with the target region, and the nick is repaired by DNA-repair machinery. Image obtained from (22) with permission. 
Figure 1.10: Schematic of integration process.

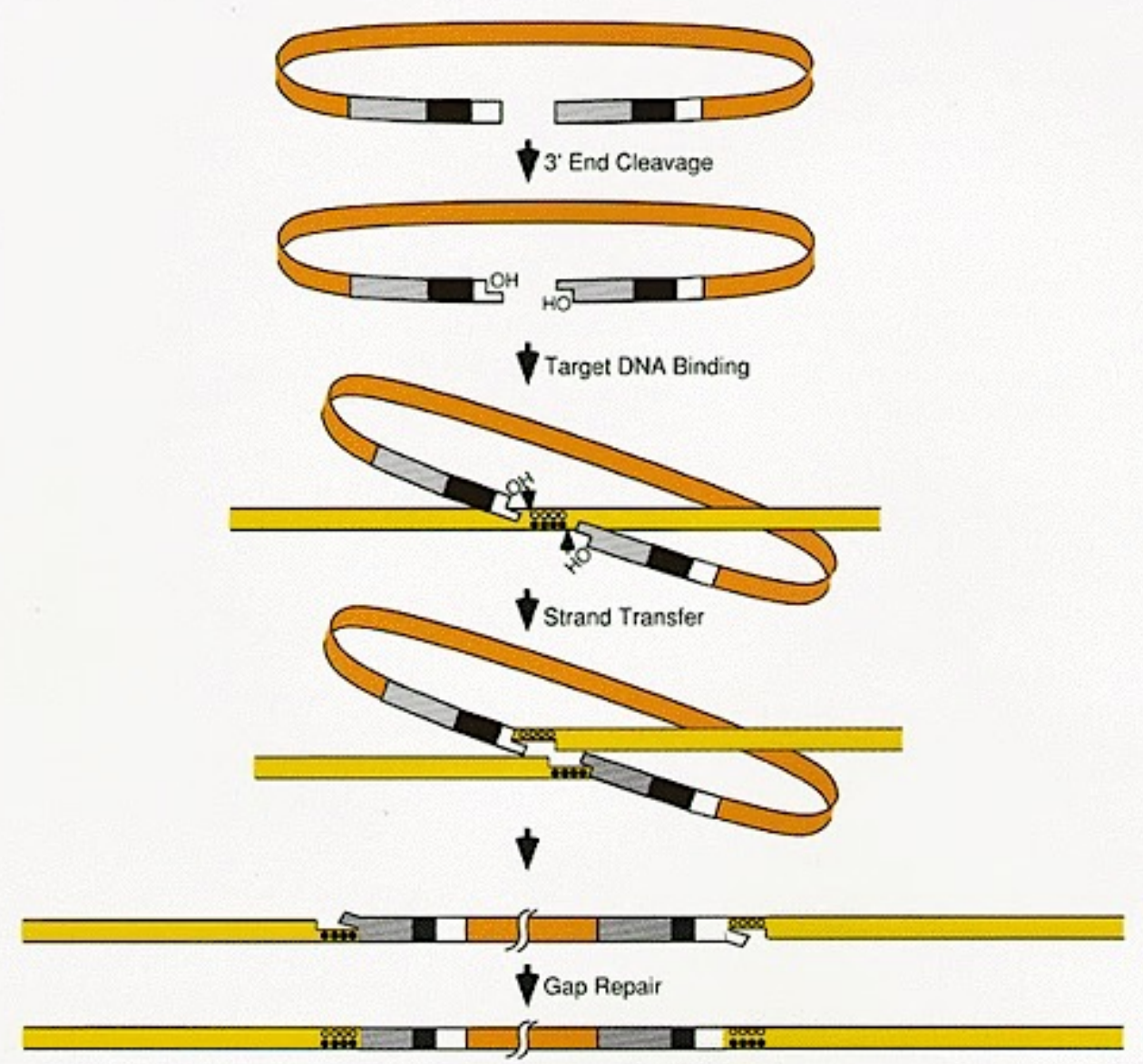


Figure 1.11: Schematic of a representative retrovirus budding.

Components in the ESCRT machinery are recruited by HIV-1 late domains to facilitate virus budding. Gag domains are depicted in yellow (MA), orange (CA), and red (NC). Recently NEDD4L is shown to be recruited to HIV-1 Gag through a cellular factor AMOT (ref). Early-acting components of ESCRT (NEDD4L, ESCRT-1, ALIX) first initiate budding process and recruit ubiquitin and late-acting components (ESCRT-III). The final component VPS4 ATPases are recruited to mediate membrane fission. Image obtained from (14) with permission (order number: 4557771276987). 
Figure 1.11: Schematic of a representative retrovirus budding.

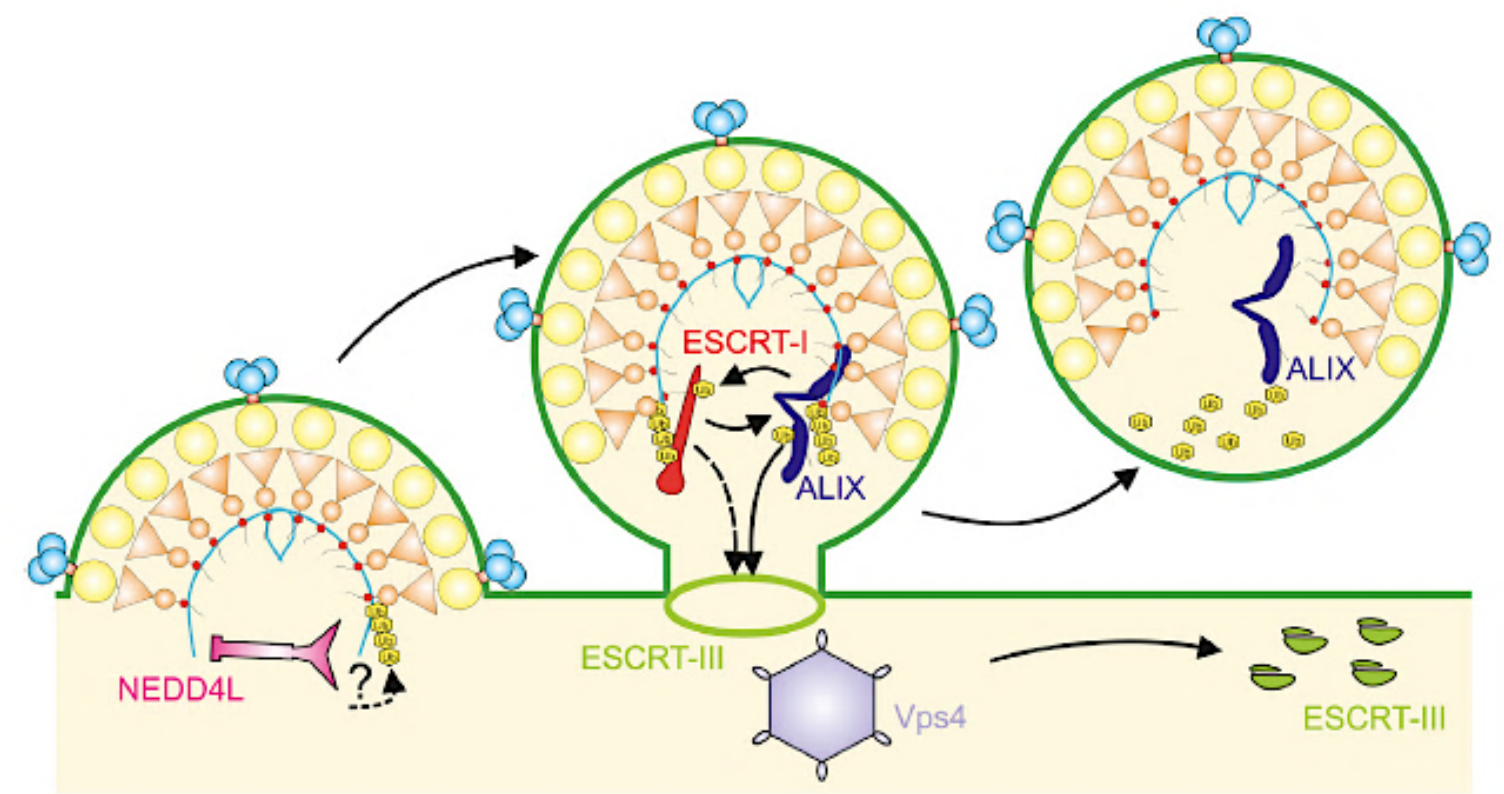




\subsection{Pseudotyping and retroviral vectors}

During viral assembly, retroviruses acquire Env glycoproteins. In some cases, retroviruses can incorporate foreign envelope glycoproteins to become infectious through a process called 'pseudotyping.' $(31,32)$ Retroviruses were shown to incorporate envelope glycoproteins from various different viral families including arenaviruses, rhabdoviruses, baculoviruses, togaviruses, and filoviruses (33-37).

Retroviral vectors have been studied as attractive gene therapy vectors, due to the efficient gene transduction. Envelope glycoproteins dictate target cell tropism, thus pseudotyping compatibility in retroviruses also largely affects production of an efficient and proper vector for gene therapy; however, the mechanism by which retroviruses incorporate envelope glycoprotein is not completely understood, even with their native Env. Four models have been hypothesized for glycoprotein incorporation process: passive incorporation, Gag and glycoprotein co-targeting to the same lipid microdomain, direct interaction, and indirect interaction between Gag and glycoproteins $(25,26)$. Each model is supported by various studies, suggesting that multiple factors can affect viral envelope glycoprotein acquisition process. Several studies focusing on Env also highlight that multiple factors affect the process from the generation to the fusogenicity of glycoproteins.

\subsubsection{Role of retroviral Env cytoplasmic tail}

The Cytoplasmic tail (CT) of the retroviral Env TM has been shown to affect various steps in Env biogenesis such as trafficking, signaling, interaction with host factors, incorporation, and fusion (38). The c-terminal cleavage of gammaretroviral Env is required for conformational change of Env to become fusogenically active during the infection, 
supporting the essential role of CT contributing the fusion (30). Retroviral CTs vary in length, suggesting a specific role of CT in diverse viruses (Figure 1.12) (38).

The Env CT of retroviruses is essential in some cases, although truncation of CT often does not affect viral replication, particularly when grown in tissue culture. Truncation of HIV-1 CTs restricted Env incorporation in most of $\mathrm{T}$ cell lines, possibly due to the absence of a trafficking motif $\mathrm{YW}_{795}(39,40)$. Gammaretroviral Env CT was also studied in depth. The trafficking motif (YXXL) was shown to be required for polarized assembly (41), while the truncation of the CT did not restrict Env recruitment on budding virus particles (42). Interestingly, the MLV Env CT was shown to dictate the selective Env incorporation in MLV particles in the presence of HIV-1 particles, suggesting a virus specific interaction of Env CT (42).

\subsubsection{Role of cellular factors}

Host cells developed various barriers to restrict and combat invading pathogens, while viruses also developed strategies to avoid the restriction mechanism as well as to utilize a host cell's machinery to propagate. The interactions between host factors and viral proteins have been targets for therapeutics, but they are also studied to understand the pathogenesis of the virus. Several studies revealed multiple restriction factors, and one of the most well-known factors is BST-2/tetherin (16). Tetherin is a major interferon-induced restriction factor in the late stage of life cycle of enveloped viruses, and it is well established the action of tetherin blocking virus budding and the counteractivity of HIV-1 accessory protein $\mathrm{Vpu}$ on tetherin. Viral release of various retroviruses tested were shown to be restricted in the presence of human tetherin (43). 
Several reports show that the HIV-1 Env CT is dispensable in certain cell lines but not others, suggesting an essential host factor involved in Env incorporation. The long CT of HIV-1 Env was shown to be indispensable for Env incorporation in more relevant cell lines, and the interaction of a Rab coupling protein FIP1C and the $\mathrm{YW}_{795}$ motif of CT was reported to be critical for incorporation $(40,44)$. Another essential trafficking motif in the Env CT is the YXXL internalization motif, which has been shown to interact with AP-2 clathrin adaptor proteins (45). Several retroviral Env CTs were reported to have this motif, and it was shown to affect trafficking, assembly, budding, and in vivo pathogenesis (41, 46-49).

\subsubsection{HIV-1 accessory protein Vpu and its mode of action on GaLV Env}

The HIV-1 accessory protein Vpu was unexpectedly found to target Gibbon Ape Leukemia Virus (GaLV) Env and restrict the pseudotyping of HIV-1 particles with GaLV Env $(50,51)$. The Vpu modulates GaLV Env through a mechanism similar to how it targets its two native targets, CD4 and tetherin. Vpu modulates CD4 degradation through the ERassociated degradation (ERAD) pathway, induced by the ubiquitination of lysine residues in CD4 CT (52). Recruitment of the degradation machinery requires two critical serine residues $\left(\mathrm{S}^{52,56}\right)$ in Vpu which are phosphorylated in cells. Vpu modulation of tetherin requires interaction between the transmembrane domains of $\mathrm{Vpu}$ and tetherin, but degradation additionally requires the $\mathrm{S}^{52,56}$ residues in the Vpu CT. However, the degradation of tetherin is not required for Vpu to enhance the viral release in certain cell lines (53-55). 
The down-modulation of GaLV Env was shown to be dependent on the GaLV Env $\mathrm{CT}$ and the $\mathrm{S}^{52,56}$ residues in the Vpu CT (51). Vpu was found to degrade GaLV Env and thus prevent GaLV Env incorporation into particles, and the structure of GaLV Env CT as well as the two critical lysine residues were shown to be required for modulation (56). In addition, sequences in the Vpu CT required to down-regulate GaLV Env and tetherin were found to largely overlap, suggesting a common down-regulating mechanism of both targets (57).

The studies presented in the following chapters focus on understanding factors that affect Env pseudotyping compatibility in different situations. The first study (chapter two) demonstrates that the co-factor $\beta \mathrm{TrCP}$ is required for HIV-1 Vpu targeting of GaLV Env as well as CD4 and tetherin. The second study (chapter three) unravels CT sequence determinants in chimeric gammaretroviral Envs dictating specific compatibility and discusses a hypothesized model of virus-specific Env compatibility. The final study (chapter four) focuses on virus production dependent on producer cell lines and attempts to dissect host factors involved in the production of two different viruses, HIV-1 and MLV. 


\section{Figure 1.12: Retroviral Env cytoplasmic tail varies in length.}

Schematic representation of retroviral TM domains of Env. TMD; transmembrane domain, HIV-1; human immunodeficiency virus-1, SIVagm; simian immunodeficiency virus from African green monkey, EIAV; equine infectious anemia virus, FIV; feline immunodeficiency virus, RSV; Rous sarcoma virus, MoMLV; Moloney-murine leukemia virus, M-PMV; Mason-Pfizer monkey virus, HTLV-1; human T-cell lymphotropic virus type 1. Figure obtained from (38) with permission (order number: 4557771447089). 
Figure 1.12: Retroviral Env cytoplasmic tail varies in length.

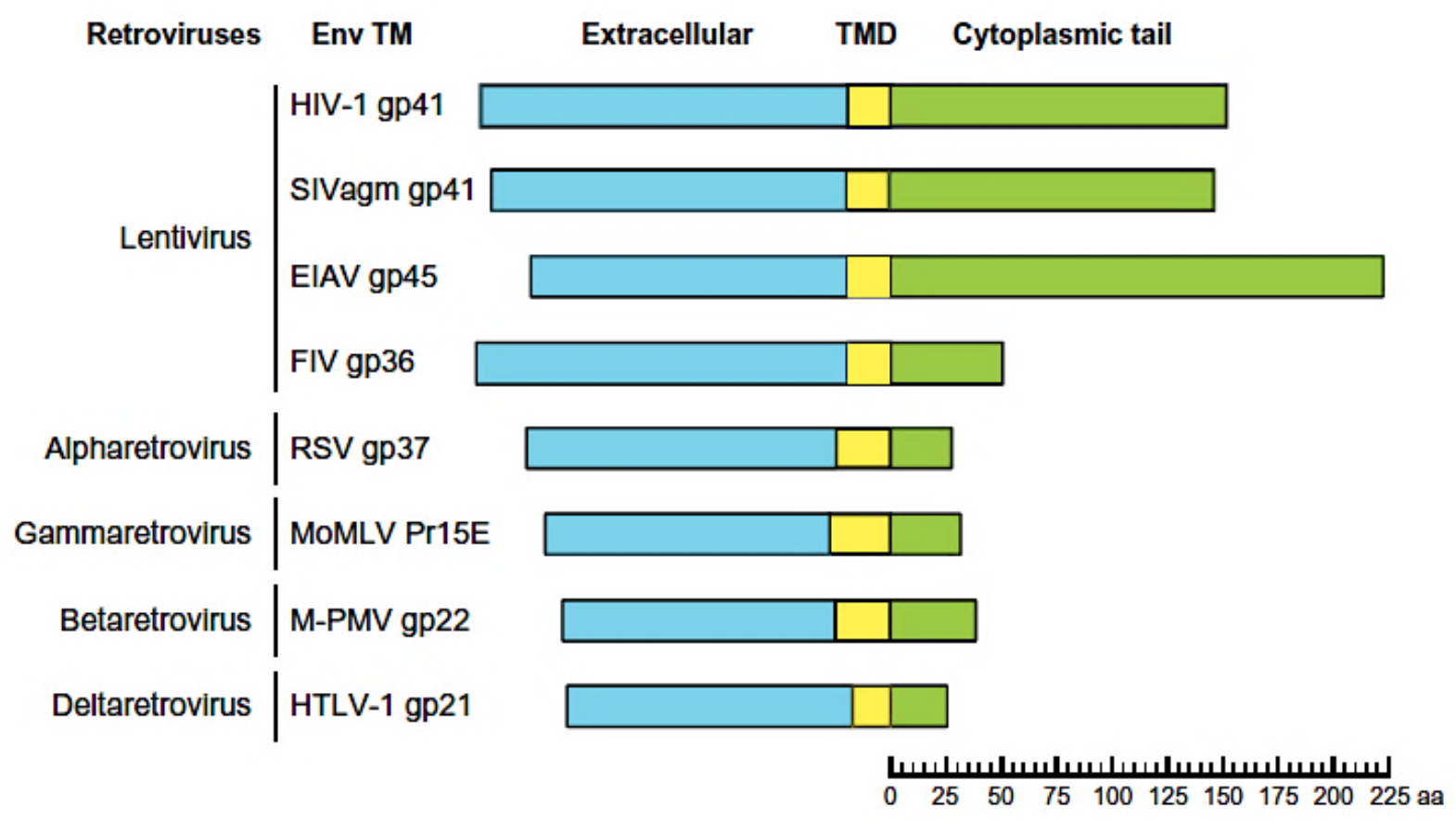




\section{II. $\beta \operatorname{TrCP}$ is Required for HIV-1 Vpu Modulation of CD4,}

\section{GaLV Env, and BST-2/Tetherin}

Yul-Eum Song ${ }^{1}$, Daniel Cyburt ${ }^{1}$, Tiffany M. Lucas ${ }^{1}$, Devon A Gregory, Terri D. Lyddon, Marc C. Johnson

1 - These authors contributed equally to this manuscript

Note: The research presented in this chapter was conducted equally between Y.E.Song, D.Cyburt, and T.M.Lucas. The manuscript was written by T.M.Lucas and D.Cyburt, and revised by all authors.

This work has been published in Viruses: $\beta$ TrCP is Required for HIV-1 Vpu Modulation of CD4, GaLV Env, and BST-2/Tetherin. Viruses 2018, 10 (10). Doi: 10.3390/v10100573. (58).

\subsection{Abstract}

The Human immunodeficiency virus-1 (HIV-1) accessory protein Vpu modulates numerous proteins, including the host proteins CD4 and BST-2/tetherin. Vpu interacts with the Skp, Cullin, F-Box (SCF) ubiquitin ligase through interactions with the F-Box protein $\beta \operatorname{TrCP}\left(1\right.$ and/or 2). This interaction is dependent on phosphorylation of $\mathrm{S}^{52,56}$ in Vpu. Mutation of $\mathrm{S}^{52,56}$, or inhibition of the SCF, abolishes most Vpu activity against CD4 and 
partly reduces activity against BST-2/tetherin. Recently, Vpu has also been reported to interact with the clathrin adapter proteins AP-1 and AP-2, and these interactions were also found to be required for BST-2/tetherin antagonism in an $\mathrm{S}^{52,56}$-dependent manner. In assays where HIV-1 is pseudotyped with gibbon ape leukemia virus (GaLV Env), Vpu has also been found to prevent GaLV Env from being incorporated into viral particles, but the mechanism for this antagonism is not fully understood. To clarify the role of the $\beta \operatorname{TrCPs}$ in Vpu function, we used CRISPR/Cas9 to generate a clonal cell line lacking both $\beta$ TrCP1 and -2 . Vpu activity against CD4 and GaLV Env was abolished in this cell line, and activity against BST-2/tetherin reduced significantly. Mutation of the $\mathrm{S}^{52,56}$ residues no longer affected Vpu activity against BST-2/tetherin in this cell line. These data suggest that the primary role of the $\mathrm{S}^{52,56}$ residues in antagonism of CD4, GaLV Env, and BST$2 /$ tetherin is to recruit the $\mathrm{SCF} / \beta \operatorname{TrCP}$ ubiquitin ligase.

\subsection{Introduction}

Human immunodeficiency virus-1 (HIV-1) Vpu is a small 81-86 amino acid accessory protein that enhances viral replication through the antagonism of a number of host cell proteins during infection $(59,60)$. Vpu has two key features that dictate functionality: an N-terminal transmembrane anchor that is capable of interacting with other transmembrane proteins, and a C-terminal cytoplasmic tail which contains two critical serine residues $\left(\mathrm{S}^{52,56}\right)$ that are phosphorylated and can dictate interaction with various cellular proteins. The best studied targets of $\mathrm{Vpu}$ are $\mathrm{CD} 4$, which is the receptor for HIV1, and BST-2/tetherin, an interferon-induced restriction factor which can prevent viral egress $(16,61,62)$. Antagonism of CD4 by Vpu occurs at the endoplasmic reticulum (ER) 
through interactions in the cytoplasmic tails of the two proteins (61). Early studies demonstrated that the membrane anchor sequence of Vpu could be scrambled without compromising the ability of $\mathrm{Vpu}$ to antagonize CD4, but changes to the cytoplasmic tail, particularly $\mathrm{S}^{52,56}$, abolished activity $(61,63-66)$. A cellular Skp, Cullim, F-box (SCF) E3 ubiquitin ligase complex is recruited by $\mathrm{Vpu}$ to target CD4 for ubiquitination and proteasomal degradation (67). The specific SCF complex utilized by Vpu contains one of two closely related F-box proteins, $\beta \operatorname{TrCP}-1$ (BTRC) or $\beta \operatorname{TrCP}-2$ (FBXW11) $(68,69)$. $\mathrm{Vpu}$ antagonism of BST-2/tetherin, on the other hand, is more nuanced as antagonism occurs through multiple steps $(16,53-55,62)$. First, Vpu directly interacts with BST$2 /$ tetherin through its transmembrane domain. This interaction is sufficient to partially displace BST-2/tetherin from the virus assembly site without downmodulating it from the cell surface, but additional domains in Vpu are required to fully counteract BST-2/tetherin $(53,55)$. Second, Vpu downmodulates BST-2/tetherin from the cell surface by preventing the trafficking of BST-2/tetherin to the plasma membrane from the trans Golgi network and/or the recycling endosome. BST-2/tetherin is constitutively endocytosed from the plasma membrane in a clathrin and adapter protein-2 (AP-2) dependent manner, but the rate of this endocytosis is not altered by $\operatorname{Vpu}(54,70-72)$. The surface downmodulation activity requires both the transmembrane anchor and the $S^{52,56}$ residues of $\mathrm{Vpu}$. While it is generally agreed that $\mathrm{Vpu}$ retains some ability to counteract tetherin in the absence of $\mathrm{S}^{52,56}(62,73,74)$, it is disputed what cellular co-factors Vpu recruits through $\mathrm{S}^{52,56}$ to facilitate surface downmodulation. While some reports suggest that $\beta \mathrm{TrCP}-1$ and -2 are partially or entirely required for this activity, others suggest that the adapter proteins AP-1 and/or AP-2 are the primary co-factors $(54,72-79)$. In addition, treatment of Vpu expressing cells with MLN4924, a compound that blocks SCF activity, does not restore 
surface BST-2/tetherin expression unless cells are first treated with interferon, suggesting that the relevant cellular cofactors vary depending on expression level $(80,81)$. Finally, Vpu targets BST-2/tetherin for degredation. Though early accounts suggested that BST$2 /$ tetherin is proteasomally degraded $(77,82)$ most reports find that the primary mechanism for degradation is through the lysosome $(54,70,73,78,83-86)$. It is generally agreed that BST-2/tetherin degradation requires both the transmembrane anchor and $S^{52,56}$, and is $\mathrm{SCF} / \beta \operatorname{TrCP}$ mediated $(54,73-75,77)$, but it is not clear how important degradation is to overall activity. Indeed, in COS cells Vpu enhances the steady state levels of BST2/tetherin while simultaneously counteracting BST-2/tetherin antiviral activity (87).

We and others identified a novel target for Vpu which is the surface viral glycoprotein from gibbon ape leukemia virus (GaLV Env) $(50,51)$. GaLV Env was found to be capable of pseudotyping HIV-1 particles when minimal packaging vectors were used (lacking accessory genes), but could not generate infectious pseudotyped particles with vectors that included Vpu. In the presence of Vpu, GaLV Env is prevented from being incorporated into particles, resulting in a 50-100 fold reduction in infectious particle production (51). In many ways Vpu appears to modulate GaLV Env using a mechanism that is similar to the modulation of CD4. Like the modulation of CD4, Vpu variants with the membrane anchor sequence scrambled are still able to modulate GaLV Env, but mutation of the $\mathrm{S}^{52,56}$ residues abolishes activity $(51,57)$. Also like with CD4, the Vpu recognition sequence in GaLV Env resides in the cytoplasmic tail, and the amino acid motif required for Vpu modulation closely resembles the motif found in the CD4 cytoplasmic tail (56). Interestingly, GaLV Env is a trimer and it was shown using functional complementation that GaLV Env can only be targeted by Vpu if all three subunits contain their cytoplasmic tail, even though the three tails do not all have to contain the Vpu 
recognition motif (88). Despite the similarities to CD4 modulation, in other ways the modulation of GaLV Env seems to use a mechanism similar to the modulation of BST2/tetherin. While CD4 is believed to be modulated in the endoplasmic reticulum (ER), BST-2/tetherin is modulated from post-ER compartments. GaLV Env is cleaved by a cellular protease in the Golgi apparatus to reach its mature form, and it is only the mature form that appears to be modulated by Vpu, suggesting that the modulation, like with BST2/tetherin, occurs in a post-ER compartment $(50,56)$. In addition, like with BST-2/tetherin, Vpu directs degradation of GaLV Env in the lysosome rather than the proteasome (50). Also like with BST-2/tetherin, Vpu is capable of preventing the incorporation of GaLV Env into viral particles without reducing the GaLV Env surface expression (51). Finally, unlike Vpu modulation of CD4 or BST-2/tetherin, GaLV Env modulation by Vpu was reported to require additional HIV-1 structural components (50).

Because Vpu has a strongly negative, rather than positive, impact on HIV-1 infectivity with GaLV Env, this combination could be adapted for use as a gain-of-function screen for inhibitors of Vpu. However, if such a screen is to be utilized, it is important to understand the mechanism of Vpu activity against GaLV Env compared to the other known Vpu targets. In this study, we sought to further characterize the mechanism of GaLV Env modulation by $\mathrm{Vpu}$ in comparison to $\mathrm{CD} 4$ and BST-2/tetherin. In particular, to examine the importance of $\beta \mathrm{TrCP}-1$ and -2 in the modulation of these proteins by generating a novel cell line using CRISPR/Cas9 technology where both proteins were eliminated. This unique tool allowed us to definitively show that $\beta \mathrm{TrCP}$ is required for $\mathrm{Vpu}$ modulation of $\mathrm{CD} 4$, GaLV Env, and BST-2/tetherin. 


\subsection{Materials and Methods}

\section{Plasmids.}

NL4-3-derived HIV-CMV-GFP was provided by Vineet KewalRamani (National Cancer Institute [NCI]-Frederick) and has been described previously (51). This proviral vector lacks the accessory genes Vif, Vpr, Nef, and Env and contains a CMV promoterdriven GFP in place of Nef. We used versions of this vector both with and without the Vpu gene (Vpu+/-). For imaging studies, a nuclear localization sequence (KKKRK) was fused to the N-terminus of the GFP sequence to allow easier enumeration of infected cells (HIV-CMV-NLS-GFP).

The ecotropic Friend MLV Env (isolate 57) expression construct and a subclone of this construct containing YFP in the protein's ectodomain were provided by Walther Mothes (Yale University) (89). The MLV/GaLV Env $\Delta 8$ and $\Delta 12$ constructs were described previously $(51,56)$. Derivatives of these constructs were generated with a hemagglutinin (HA) tag or mCherry tag in place of the YFP sequence. The cherry tagged GaLV Env $\Delta 8$ was subcloned into retroviral transfer vector PQCXIH (Clontech, Mountain View, CA, USA) for generation of a stable cell line. The pSpCas9(BB)-2A-Puro (PX459) V2.0 was a gift from Feng Zhang (Addgene plasmid \# 62988) (90). The $\beta \operatorname{TrCP}-1$ and -2 guide RNA (gRNA) sequences (GTAATAATGGCGAACCCCCT, and GACACGGCGTAAAGTGATGT, respectively) were synthesized, and inserted into the BsmBI restriction site in PX459. The Ub-K0 construct was provided by Mark Hannink (University of Missouri). The dominant negative (DN) $\beta \operatorname{TrCP}$ and the Vpu-GFP constructs were provided by Ed Stephens (University of Kansas Medical Center) (54, 91). The pHAGE DN Cullin1 was a gift from Stephen Elledge (Addgene plasmid \# 41911) (92). Vpu-IRES-GFP and pcDNA3.1 human CD4 plasmids were kindly provided by Frank 
Kirchoff (University of Ulm, Germany) (93). For western blotting, hemagglutinin (HA) tag (YPYDVPDYA) was inserted in the N-terminal of CD4 immediately after the signal peptide. Human Tetherin-HA plasmid was kindly provided by Paul Bieniasz (94). The retroviral transfer vector for stably expressing human $\beta \operatorname{TrCP}-2$ was obtained by amplifying $\beta$ TrCP-2 from human cDNA collected from 293FT cells cloning it into vector pQCXIH (Clontech).

\section{Cell Lines.}

The 293FT cell line was obtained from Invitrogen (Carlsbad, CA, USA). This cell line is a HEK293 derivative similar to 293T, which has been adapted for high transfectability. The $\beta \operatorname{TrCP}$ double knockout cell lines were obtained by transfecting the 293FT cells with PX459 plasmids encoding $\beta \operatorname{TrCP} 1$ and $\beta \operatorname{TrCP} 2$ gRNA described in plasmids section. Single cell isolates from the transfected population were screened by transfecting clonal isolates with HIV-CMV-GFP $\left(\mathrm{Vpu}^{+}\right)$and GaLV Env and testing if infectious particles are produced. Isolates that were able to produce infectious particles under these conditions were further screened by amplifying and sequencing the sequence flanking the Cas9/gRNA target site. $\beta \operatorname{TrCP} 1 / 2$ knockout cells with $\beta \operatorname{TrCP} 2$ add back were obtained by transducing the knockout cell line with an MLV based vector coding for both a hygromycin resistance gene and $\beta \operatorname{TrCP} 2$ (amplified from 293FT cell cDNA); the transduced population was subsequently selected with hygromycin. The mCherry tagged-GaLV Env $\Delta 8$ expression cell line was produced the same way except 293 TVA cells were used (95). These cells were chosen because they are a cell line our lab routinely uses which are morphologically more suitable for imaging studies than 293FT cells. While both cell lines are HEK293 derivatives, 293FT cells tend to grow in clumps and adhere poorly to coverslips, whereas 
293 TVA cells (like 293T cells) appear much flatter and adhere to coverslips much more firmly. The difference is not believed to be a result of the TVA transgene as most HEK293 derivatives, with the exception of 293FT, have a morphology similar to 293 TVA cells. Following selection, cells were sorted using a Beckman Coulter MoFlo XDP (Cell and Immunobiology Core, University of Missouri) for cells that express high levels of mCherry. This cell population was transduced with HIV-CMV-NLS-GFP $\left(\mathrm{Vpu}^{+/}\right)$at a relatively low $(<2)$ multiplicity of infection (MOI). The cell line expressing the ecotropic F-MLV Env receptor, 293T mCAT-1, was provided by Walther Mothes. All cell lines were maintained in Dulbecco's modified Eagle media (DMEM), containing 10\% fetal bovine serum, $2 \mathrm{mM}$ L-glutamine, $1 \mathrm{mM}$ sodium pyruvate, $10 \mathrm{mM}$ nonessential amino acids and $10 \mathrm{mM}$ MEM Vitamins.

\section{Western Blotting.}

For inhibitor studies on protein expression, 293FT cells were transfected with 500 ng HA-tagged GaLV Env $\Delta 8,25 \mathrm{ng}$ Vpu-GFP and $200 \mathrm{ng}$ empty-plasmid. At $16 \mathrm{~h}$ posttransfection, cells were treated for $7 \mathrm{~h}$ with either a DMSO control, proteasomal inhibitor cocktail $(10 \mu \mathrm{M}$ MG132 and $20 \mu \mathrm{M}$ epoximicin) or a lysosomal inhibitor (100 nM Bafilomycin A1). Cellular 293FT lysate was collected and treated with PNGase as previously described (50). Lysate protein concentration were determined using a Bradford reagent against a BSA standard and $10 \mu \mathrm{g}$ of protein was resolved by SDS-PAGE and immunoblotted with anti-HA (Sigma H3663, 1:1,000, St. Louis, MO, USA) followed by anti-mouse HRP (Sigma A5278, 1:10,000). Following transfer, blots were stained with Ponceau S to confirm protein transfer and equivalent loading. For CD4 expression, 293FT cells and its derivatives were transfected with $100 \mathrm{ng}$ of HA-tagged CD4 and $500 \mathrm{ng}$ of 
either 500 ng Vpu-IRES-GFP or 500 ng IRES-GFP. Cellular lysate was collected after 2 days and $15 \%$ of whole cell lysate was resolved by SDS-PAGE and immunoblotted. CD4 expression was detected by anti-HA, and GAPDH was detected for loading control (Santa Cruz Biotechnology SC-47724, 1:500, Dallas, TX, USA).

\section{Infectivity Assays.}

Except where noted, infectivity assays were performed by transfecting 293FT cells or its derivatives with the Env defective HIV-CMV-GFP proviruses. All transfections were performed in $35 \mathrm{~mm}$ dishes using polyethylenimine (PEI) (96). Virus containing media was frozen at $-80^{\circ} \mathrm{C}$ prior to infections in $293 \mathrm{~T}$ mCAT- 1 cells. Transduced cells were fixed with paraformaldehyde two days post-transduction, and analyzed on an Accuri flow cytometer. For proteasomal and lysosomal inhibitor studies, cells were transfected with $600 \mathrm{ng}$ of HIV-CMV-GFP (Vpu+/-) and $400 \mathrm{ng}$ GaLV Env $\Delta 8$ or GaLV Env $\Delta 12$. Cells were treated $40 \mathrm{~h}$ post-transfection with either proteasomal inhibitors ( $20 \mu \mathrm{M}$ ALLN or $10 \mu \mathrm{M}$ MG132), lysosomal inhibitors $100 \mathrm{nM}$ Bafilomycin A1 (BafA) or $100 \mathrm{nM}$ Concanamycin A (ConA), a DMSO control or no treatment (NT). Virus-containing culture media was collected $12 \mathrm{~h}$ later and spinoculated onto $293 \mathrm{~T}$ mCAT-1 cells at $30^{\circ} \mathrm{C}$ for 30 min. For infectivity with the mCherry-GaLV Env $\Delta 8$ transduced cell line, both the virus producing cells and the virus containing media were collected. The cells were fixed and analyzed using an Accuri flow cytometer to determine the number of transduced (GFP expressing) cells and the viral media was used to transduce $293 \mathrm{~T}$ mCAT-1 cells. The output

was expressed as the number of infectious units per $10^{6}$ virus producing cells. For polyubiquitin experiments, cells were transfected with $250 \mathrm{ng}$ of $\mathrm{HIV}-\mathrm{CMV}-\mathrm{GFP}\left(\mathrm{Vpu}^{+/}\right)$, 250 ng GaLV Env $\Delta 8$ or GaLV Env $\Delta 12$ and 500 ng dominant-negative ubiquitin (Ub- 
K0). For Neddylation inhibitor studies, cells were transfected with $900 \mathrm{ng}$ of provirus and $100 \mathrm{ng}$ of MLV Env or GaLV Env $\Delta 8$. The next day, cells were treated with $1 \mu \mathrm{M}$ MLN4924 and viral media was collected $24 \mathrm{~h}$ later. For DN $\beta \operatorname{TrCP}$ and DN Cullin1 studies, cells were transfected with $500 \mathrm{ng}$ of provirus, $100 \mathrm{ng}$ of MLV Env or GaLV Env $\Delta 8$ and $500 \mathrm{ng}$ or $100 \mathrm{ng}$ of $\mathrm{DN} \beta \operatorname{TrCP}$ and $\mathrm{DN}$ Cullin1, respectively. For experiments in the knockout cell lines, cells were transfected with $900 \mathrm{ng}$ of HIV-CMV-GFP $\left(\mathrm{Vpu}^{+/}\right), 100 \mathrm{ng}$ of MLV Env or GaLV Env $\Delta 8$. For BST/tetherin activity assays, cells were transfected with $900 \mathrm{ng}$ of HIV-CMV-GFP $\left(\mathrm{Vpu}^{++}\right), 100 \mathrm{ng}$ of VSV-G and 0-80 $\mathrm{ng}$ of BST-2/tetherin.

\section{Surface Labeling.}

For CD4 surface labeling, 293FT cells and derivatives were plated in $35 \mathrm{~mm}$ dishes and transfected the following day with $100 \mathrm{ng}$ human CD4 and either $500 \mathrm{ng}$ Vpu-IRESGFP or 500 ng IRES-GFP. Where appropriate, wells were transfected with varying amounts of DN $\beta \operatorname{TrCP}(500 \mathrm{ng}$ ) or DN Cullin1 (100ng). For experiments employing MLN4924, media was changed 24 hours post-transfection and replaced with media containing 1 uM MLN4924 in DMSO; surface staining was performed 24 hours post-media change. For surface staining, cells were lifted from the plates using PBS with $10 \mathrm{mM}$ EDTA, blocked with 5\% goat serum in PBS for 30 min, and incubated in 96 well U-bottom plates for $1 \mathrm{~h}$ with $1 \%$ anti-CD4 APC conjugate primary antibody in PBS/EDTA with $1 \%$ goat serum. After staining, cells were washed with PBS and fixed with $1 \%$ paraformaldehyde. Surface staining of transfected cells was analyzed the same day on an Accuri C6 flow cytometer. CD4 surface staining was read as the mean fluorescence intensity of the far-red APC signal among the GFP-transfected cell population. For mCherry-GaLV Env $\Delta 8$ surface labeling, cells were first blocked with $5 \%$ goat serum in 
PBS for 30 minutes, incubated with primary anti-mCherry rabbit antibody (abcam ab167453) diluted 1:500 in PBS for 1 hour at $4^{\circ} \mathrm{C}$. After incubation, cells were washed 23 times with PBS and fixed in 4\% paraformaldehyde for $20 \mathrm{~min}$, and washed again 2-3 times with PBS. Cells were then blocked again with 5\% goat serum in PBS for 30 min and incubated in secondary anti rabbit Alexa 405 antibody (Life Technologies, Carlsbad, CA, USA) in $10 \mu \mathrm{g} / \mathrm{ml}$ concentration for 1 hour at $4^{\circ} \mathrm{C}$. Cells were finally washed with PBS 23 times and analyzed on flow cytometer (BD LSRFortessa X-20, San Jose, CA, USA) at the Cell and Immunobiology Core at the University of Missouri, and analyzed with flowJo software (version 10.3, FlowJo, LLC, Ashland, OR, USA).

\section{Imaging.}

For mCherry-GaLV Env $\Delta 8$ imaging, cells were plated on a glass bottom dish (MatTek, Ashland, MA, USA). Cells were first blocked with 5\% goat serum in PBS for 30 min, incubated with primary anti-mCherry rabbit antibody (abcam ab167453) diluted 1:500 in PBS for $1 \mathrm{~h}$ at $4^{\circ} \mathrm{C}$. After incubation, cells were washed 2-3 times with PBS and fixed in 4\% paraformaldehyde for 20 min, and washed again 2-3 times with PBS. Cells were then blocked again with 5\% goat serum in PBS for 30 min and incubated in secondary anti rabbit Alexa 405 antibody (Life Technologies) in $10 \mu \mathrm{g} / \mathrm{ml}$ concentration for 1 hour at $4^{\circ} \mathrm{C}$. Cells were finally washed with PBS 2-3 times. Cells were imaged on an Olympus IX81 with 100X 1.4 oil objective with deconvolution processing of Olympus CellSens Dimension software (version 1.3, Olympus, Shinjuku, Tokyo, Japan). Images were adjusted using ImageJ software to highlight the different intensity of signal. 


\subsection{Results}

The glycoprotein used for analyzing the mechanism of Vpu action in this study is a chimeric ecotropic MLV Env glycoprotein that contained the C-terminal cytoplasmic tail from GaLV Env with the last 8 amino acids deleted (henceforth referred to as GaLV Env $\Delta 8)$. This glycoprotein was chosen because we showed previously that it forms infectious particles with HIV-1 much more efficiently than wildtype GaLV Env, but is still potently prevented from being incorporated into HIV-1 particles by Vpu (56). Infectious HIV-1 particle production with GaLV Env $\Delta 8$ correlates with incorporation into viral particles, making loss of infectious particle production a quantitative measure of Vpu activity.

\section{GaLV Env $\Delta 8$ is targeted for lysosomal degradation.}

We first sought to evaluate the effect of Vpu on the steady-state levels of GaLV Env $\Delta 8$. Cells were transfected with GaLV Env $\Delta 8$ along with an independent Vpu expression plasmid and GaLV Env expression in cell lysates was measured by western blot. As reported previously, expression of Vpu did not alter the level of the uncleaved Env precursor protein (Pr85), but greatly reduced the amount of mature cleaved (gp70) Env protein $(50,56)$ (Figure 2.1A). Because retroviral Env proteins are not cleaved until they pass through the Golgi apparatus (97), this is consistent with GaLV Env $\Delta 8$ being targeted by $\mathrm{Vpu}$ in a post-ER compartment. HIV-1 Vpu targets CD4 for degradation through proteasomal degradation (67), but targets BST-2/tetherin through lysosomal degradation $(54,78)$. HIV-1 Vpu was previously reported mediate lysosomal degradation of GaLV Env (50). To verify that the GaLV Env construct used in this study behaves the same as the full-length protein used previously, transfected cells were treated with lysosomal or proteasomal inhibitors. Treatment of cells with lysosomal inhibitor Bafilomycin A 
restored mature Env in cells, but treatment with proteasome inhibitors did not (Figure 2.1A). These results are consistent with the previous report; however, unlike the previous report (50), we found that Vpu was sufficient to modulate the mature GaLV Env expression level and did not require the presence of additional HIV-1 structural proteins.

\section{Inhibition of lysosomal degradation does not restore infectious particle production with GaLV Env $\Delta 8$.}

We next asked whether lysosomal inhibition was sufficient to restore infectious particle production with GaLV Env $\Delta 8$. For this experiment, an NL4-3 derived HIV-1 provirus (Vpu+/-) lacking Env, Vif, and Vpr and containing CMV-GFP in place of Nef (henceforth HIV-CMV-GFP) was pseudotyped with GaLV Env $\Delta 8$ and infectious particle output was measured following treatment with proteasomal or lysosomal inhibitors. As a control, the same proviruses were pseudotyped with GaLV Env $\Delta 12$, which has four additional amino acids deleted rendering the protein insensitive to $\mathrm{Vpu}$ (56). As expected, Vpu reduced the infectious particle production with GaLV Env $\Delta 8$ by approximately 100 fold, but had no effect on the infectious unit production with Vpu-insensitive GaLV Env $\Delta 12$ (Figure 2.1B). Neither proteasomal nor lysosomal inhibitors restored infectious particle production to HIV-1 particles pseudotyped with GaLV Env $\Delta 8$. Proteasomal inhibitors noticeably reduced the production of infectious viral particles, but it did so with both glycoproteins and was not affected by Vpu. Thus, preventing GaLV Env $\Delta 8$ degradation was not sufficient to restore infectious particle production. A possible explanation for this is that the GaLV Env protein is sequestered in the lysosome and away from viral particles, even if it is not degraded at the lysosome. A similar phenomenon has been observed with Vpu modulation of BST-2/tetherin in COS cells (87). 


\section{Loss of surface expression weakly correlates with loss of infectious particle}

production.

We demonstrated previously that loss of infectious particle production with GaLV Env in the presence of $\mathrm{Vpu}$ did not require noticeable surface downmodulation (51). However, these previous studies were performed in transfected cells in which both Vpu and GaLV Env were presumably overexpressed, and the study only addressed surface expression. To study the change in GaLV Env distribution using a system where the

protein is not as highly expressed, we first generated a novel cell line expressing a fluorescently tagged version of GaLV Env $\Delta 8$ that contained mCherry cloned into the proline rich ectodomain of the glycoprotein. This construct was chosen because it produces infectious particles with similar efficiency to untagged protein, and it remains sensitive to Vpu expression. Next, we transduced these cells at a low MOI $(<2)$ with HIV-CMV-GFP particles $(\mathrm{Vpu}+/-)$ and analyzed total Env expression, Env distribution, surface Env expression, and infectious particle production (Figure 2.2). Transduced and un-transduced cells displayed discrete Env punctae within the cytoplasm of cells, but only weak signal at the plasma membrane. There was no obvious difference in quantity or distribution in the mCherry signal in cells transduced with Vpu containing provirus. However, when we stained surface Env expression using an antibody against mCherry, there was a noticeable loss in surface Env expression in Vpu expressing cells relative to neighboring untransduced cells (Figure 2.2). To quantitate this difference, the cells were collected and analyzed by flow cytometry. There was no quantifiable reduction in total mCherry expression in cells expressing Vpu. However, when surface labeling was quantified there was a consistent 23 fold reduction in surface expression relative to cells transduced with virus lacking Vpu. It was surprising that $\mathrm{Vpu}$ did not cause a quantifiable difference in overall GaLV Env 
expression levels, which is in contrast to what we and others have observed in experiments where GaLV Env is transfected into cells. We believe that the difference is because in the stable cells where Env is expressed at low level, which may make Vpu modulation less apparent. Alternatively, the difference could simply reflect differences in the two assays. While western blot (Figure 2.1A) detects absolute protein levels, the flow cytometry readings (Figure 2.2B) detect the fluorescent signal from the produced protein. To determine the infectious pseudotyped particle production from these cells, the supernatant was collected and used to transduce fresh cells. Because the Env expressing cells do not express the mCAT-1 receptor, infectivity could only be scored on a secondary cell line that expresses the ecotropic MLV Env receptor mCAT-1 (293T mCAT). While both transduced cell lines produced infectious particles, the output from cells transduced with the Vpu-containing provirus was 50-100 times lower (Figure 2.2D). Thus, while Vpu only partially downmodulates GaLV Env $\Delta 8$ from the surface of cells, it almost completely abrogates production of infectious particles.

\section{Vpu inhibition of GaLV Env $\Delta 8$ infectious particle production is polyubiquitin dependent.}

Because some Vpu activities involve polyubiquitination, we employed a dominant negative ubiquitin to address whether polyubiquitination is required to restrict infectious particle production. This dominant negative protein has all seven of its lysine residues mutated to arginine (Ub-K0) such that the protein can be covalently added to substrates, but cannot form ubiquitin chains due to its lack of lysine acceptor residues (98-102). We found Ub-K0 enhanced infectious particle output of HIV-CMV-GFP (Vpu+) approximately 100 -fold with GaLV Env $\Delta 8$, but it had no effect on HIV-CMV-GFP (Vpu-) 
particles (Figure 2.3). Ub-K0 had no effect with GaLV Env $\Delta 12$ in the presence or absence of $\mathrm{Vpu}$. These findings demonstrate that polyubiquitination is required for the modulation of GaLV Env $\Delta 8$ by Vpu.

\section{Vpu inhibition of GaLV Env $\Delta 8$ infectious particle production is restored by neddylation inhibitor MLN4924.}

The dependence on both polyubiquitin chains (Figure 2.3) and the Vpu $\mathrm{S}^{52,56}$ residues (51) indicated that Vpu may be hijacking and utilizing a ubiquitin ligase to prevent mature GaLV trafficking to viral particles. MLN4924 is an ATP analogue that binds to and irreversibly inhibits the Nedd8 activating enzyme, a member of a pathway responsible for crosslinking a Nedd8 moiety onto Cullin proteins and critically important for their activity (103). As such, MLN4924 is a strong inhibitor of all Cullin-containing E3 ubiquitin ligases in the cell (104). To determine if Cullin activity is required for Vpu activity against GaLV Env $\Delta 8$, we transfected HIV-CMV-GFP ( $\mathrm{Vpu}+/$-) along with GaLV Env $\Delta 8$ or Vpu-resistant MLV Env, and measured infectious particle output in the presence or absence of MLN4924 (Figure 2.4A). Because of variation in infectious particle production among glycoproteins, the infectious particle output for each set of experiments is normalized and expressed as a percent compared to the Vpu- provirus with no treatment. Infectious output from particles generated with MLV Env was not altered by Vpu expression and was modestly inhibited by MLN4924. As expected, infectious particle output from cells expressing GaLV Env $\Delta 8$ was severely restricted by Vpu. However, this Vpu inhibition was eliminated by MLN4924 treatment. Thus, Vpu antagonism of GaLV Env is dependent on a cellular Cullin-containing ubiquitin ligase enzyme. 


\section{Vpu inhibition of GaLV Env $\Delta 8$ infectious particle production is restored by dominant negative (DN) Cullin 1, but not a DN $\beta$ TrCP.}

Since Vpu is known to hijack SCF- $\beta$ TrCP, we used two DN proteins to test if $\mathrm{Vpu}$ antagonism of GaLV Env $\Delta 8$ was dependent on Cullin1 and the F-box proteins $\beta$ TrCP-1/2. DN Cullin1 is missing its Rbx domain, through which it binds to and engages the E2 ubiquitination machinery; as such, it binds to the Skp/F-box protein complex but does not support ubiquitin transfer to the target substrate protein. DN $\beta \operatorname{TrCP}$ is missing its F-box domain, through which it contacts the Skp1/Cullin1/E2 ligase machinery complex, so it will bind to substrate proteins and $\mathrm{Vpu}$, but cannot support ubiquitin transfer to target protein, and therefore blocks Vpu activity with both $\beta \operatorname{TrCP}-1$ and $-2(54,68)$. We cotransfected HIV-CMV-GFP (Vpu+/-) with MLV/GaLV $\Delta 8$ Env or MLV Env along with DN Cullin1, DN $\beta$ TrCP or an empty control vector (Figure 2.4B). Both DN proteins reduced infectious particle production 2-3 fold with MLV Env regardless of Vpu expression, as well as MLV/GaLV $\Delta 8$ particles in the absence of Vpu. This is most likely due to off-target effects of the expressed proteins. Once again, infectious particle production with MLV GaLV Env $\Delta 8$ was severely restricted (50-100 fold) in the presence of Vpu. Curiously, dominant negative Cullin1 potently rescued MLV/GaLV $\Delta 8$ infectious particle production, while $\mathrm{DN} \beta \mathrm{TrCP}$ showed no rescue at all (Figure 2.4B). This experiment was repeated numerous times with both higher and lower DN $\beta \operatorname{TrCP}$ concentrations, but infectious particle rescue was never observed. SCF ligases utilize over 80 possible F-box proteins and these findings raised the possibility that Vpu is capable of hijacking F-box proteins other than $\beta \mathrm{TrCP}$, expanding the network of potential E3 enzymes it could subvert. To confirm that the DN $\beta$ TrCP expression construct was functional, we 
confirmed that it was capable of restoring CD4 surface expression in the presence of Vpu (Figure 2.4C).

\section{$\beta \operatorname{TrCP}$ is required for $\mathrm{Vpu}$ activity.}

To unambiguously determine the $\beta \operatorname{TrCP}$-dependency of Vpu activity, we chose to use CRISPR/Cas9 to generate a novel 293FT cell line with both $\beta \operatorname{TrCP}-1$ and -2 knocked out. CRISPR/Cas9 gRNAs were designed against both genes and introduced into cells. To our surprise, the pool of CRISPR treated cells showed some enhancement in infectious particle production with GaLV Env in the presence of Vpu (not shown), suggesting that $\beta \operatorname{TrCP}$ is at least partially required for activity. Clonal isolates were obtained from this population of cells by limiting dilution and cell lines were screened for enhanced infectious particle production with GaLV Env in the presence of Vpu. Because we could not identify any commercial antibodies that reliably detect the two $\beta \operatorname{TrCP}$ proteins, clonal isolates were further screened by recovering the CRISPR gRNA target sites by PCR and sequencing the product to confirm the knockout $(\mathrm{KO})$ at each locus (Figure 2.5). The clonal isolate selected $(\beta \operatorname{TrCP}-1 / 2 \mathrm{KO})$ contained a homologous -2 indel in $\beta \operatorname{TrCP}-1$ locus, and a mixture of $-2,-16,+62$ indels in the $\beta \operatorname{TrCP}-2$ locus. These three indels persisted through multiple rounds of clonal isolation, suggesting that the cell line is at least trisomic at this locus, which is on chromosome 5. To rule out any changes in this cell line being a result of off-target CRISPR effects, we stably reintroduced $\beta \operatorname{TrCP}-2$ to the cell line to examine in parallel ( $\beta \operatorname{TrCP}-1 / 2 \mathrm{KO}+\beta \operatorname{TrCP}-2$, henceforth referred to as 'add back').

We first tested the $\beta \operatorname{TrCP}-1 / 2 \mathrm{KO}$ and add back cell lines in infectivity assays with

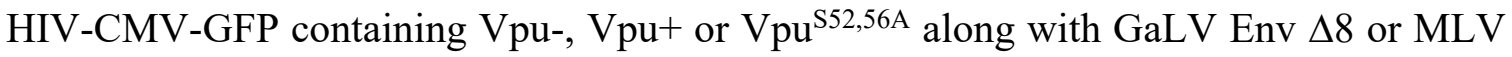


Env (Figure 2.6A). Because the three cell lines did not transfect at exactly the same rates, the infectious particle production from each cell line was expressed relative to that from the Vpu- provirus. In both the parental 293FT cell line and the add back cell line the presence of wildtype Vpu greatly reduced infectious particle production (10-fold or more) with GaLV Env $\Delta 8$, but $\mathrm{Vpu}^{\mathrm{S} 52,56 \mathrm{~A}}$ had little to no effect (2-fold or less). Surprisingly, we found that in the $\beta \operatorname{TrCP}-1 / 2 \mathrm{KO}$ cell line Vpu lost the ability to restrict GaLV Env $\Delta 8$, and

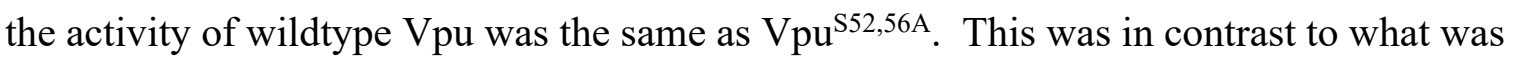
observed with the DN $\beta \operatorname{TrCP}$ (Figure 2.4B).

Next, we examined the ability of Vpu to downmodulate CD4 in these cell lines. As would be expected, in the parent 293FT cell line and in the add back cell line Vpu greatly reduced surface CD4 expression. In fact, each time the experiment was performed Vpu appeared to downmodulate CD4 more efficiently in the add back cell line than in the parent cell line. This could likely be due to $\beta \operatorname{TrCP}-2$ expression being higher in the add back cell line than in the parent cell line (Figure 2.6B). However, in the $\beta \operatorname{TrCP}-1 / 2 \mathrm{KO}$ cell line Vpu was not able to meaningfully downmodulate CD4 expression. When analyzed by western, the steady state levels of CD4 decreased in the presence of Vpu in 293FT and add back cells, but in the $\beta \operatorname{TrCP}-1 / 2 \mathrm{KO}$ cells the $\mathrm{CD} 4$ expression level increased in the presence of $\mathrm{Vpu}$ (Figure 2.6C). This confirms that $\beta \operatorname{TrCP}$ is required for $\mathrm{CD} 4$ degradation by Vpu.

Finally, we chose to examine the antagonism of BST-2/tetherin by Vpu in these cell lines (Figure 2.6D). For this experiment each cell line was transfected with HIVCMV-GFP containing Vpu-, Vpu+ or $\mathrm{Vpu}^{\mathrm{S} 52,56 \mathrm{~A}}$ and a Vpu-insensitive glycoprotein along with $0,10,20,40$, or $80 \mathrm{ng}$ of a tetherin expression plasmid. As expected, in the 293FT parent cell line infectious particle output was essentially abolished with $10 \mathrm{ng}$ of tetherin, 
but the $\mathrm{Vpu}+$ infectious particle output was only partially reduced. The infectious particle output with $\mathrm{Vpu}^{\mathrm{S} 52,56 \mathrm{~A}}$ was higher than with $\mathrm{Vpu}-$, but there was a very clear and statistically significant difference between wildtype $\mathrm{Vpu}$ and $\mathrm{Vpu}^{\mathrm{S} 52,56 \mathrm{~A}}$. The results were largely the same in the add back cell line except wildtype Vpu appeared to be more effective at countering tetherin than in the parent cell line. This is similar to what was observed in the CD4 modulation experiments and are consistent with $\beta \mathrm{TrCP}-2$ being able to enhance Vpu activity. The output with the $\beta \operatorname{TrCP}-1 / 2 \mathrm{KO}$ cell line was clearly different. The provirus with $\mathrm{Vpu}^{\mathrm{S} 52,56 \mathrm{~A}}$ was still able to counter tetherin as efficiently as in the parent and add back cell lines, but the provirus with wildtype Vpu had greatly diminished activity. There was a small (0.5-fold) but statistically significant enhancement of wildtype Vpu activity over $\mathrm{Vpu}^{\mathrm{S52,56 \textrm {A }}}$ at $10 \mathrm{ng}$ of tetherin, but at all other concentrations there was no statistically significant difference between wildtype $\mathrm{Vpu}$ and $\mathrm{Vpu}^{\mathrm{S} 52,56 \mathrm{~A}}$. We conclude that in this cell line, the predominant function of the $\mathrm{S}^{52,56}$ residues in $\mathrm{Vpu}$ is to recruit $\beta \operatorname{TrCP}$.

\subsection{Discussion}

Here we verified the role of the $\mathrm{SCF} / \beta \operatorname{TrCP} 1 / 2 \mathrm{E} 3$ ubiquitin ligase as a critical cofactor for Vpu to block incorporation of mature, functional GaLV Env into pseudotyped HIV-1 particles. We further confirmed that this ligase is also critical for Vpu to downmodulate CD4 from the cell surface and to antagonize the host restriction factor BST$2 /$ tetherin. While there has generally been a consensus that $\beta \operatorname{TrCP}$ is the critical host factor required to promote $\mathrm{CD} 4$ downmodulation, the role of $\mathrm{SCF} / \beta \operatorname{TrCP}$ in $\mathrm{BST}-2 /$ tetherin antagonism has been more controversial $(74,75,80)$. Initial studies on the mechanism of how Vpu blocks GaLV Env incorporation into virions suggested that it was at least similar 
to the CD4 mechanism, since the two proteins share the same Vpu sensitivity motif in the cytoplasmic tail (56). However, the dominant negative version of $\beta \operatorname{TrCP}$, which blocked Vpu antagonism of CD4, failed to block its activity against GaLV Env. Therefore the finding that Vpu activity against GaLV Env was lost in $\beta \operatorname{TrCP}-1 / 2 \mathrm{KO}$ cell line was surprising, but clearly showed that $\beta \mathrm{TrCP}$ was required for $\mathrm{Vpu}$ to block formation of GaLV Env pseudotypes. This is not the first report of the $\mathrm{DN} / \beta \operatorname{TrCP}$ yielding a different outcome than knocking down $\beta \operatorname{TrCP}-1$ and -2 . Tervo et al. demonstrated that DN $\beta \operatorname{TrCP}$ could restore CD4 and BST-2/tetherin surface expression in the presence of Vpu, but it could not restore overall BST-2/tetherin expression levels (74). In contrast, when they knocked down $\beta \operatorname{TrCP}-1$ and -2 in the presence of Vpu it restored BST-2/tetherin expression levels. The authors speculated that the DN $\beta \operatorname{TrCP}$ may impair the Vpu-dependent proteasomal degradation of Vpu targets, but may not be able to prevent these targets from trafficking to the lysosome. The simplest explanation for the failure of $\mathrm{DN} \beta \operatorname{TrCP}$ to restore GaLV Env infectious particle production in the presence of Vpu is that DN $\beta \operatorname{TrCP}$ has off-target effects that distort the assay. In particular, we found that the concentrations of $\mathrm{DN} \beta \operatorname{TrCP}$ required to restore $\mathrm{CD} 4$ surface expression in the presence of $\mathrm{Vpu}$ inhibited infectious particle production even in the absence of Vpu (Figure 2.4). In the data shown, the same concentration of DN $\beta \operatorname{TrCP}$ was used in both the CD4 and GaLV Env assays. Lower concentrations of $\mathrm{DN} \beta \operatorname{TrCP}$ was less detrimental to infectivity, but was also less effective at restoring CD4 expression in the presence of $\mathrm{Vpu}$. DN $\beta \operatorname{TrCP}$ was unable to enhance GaLV Env infectivity in the presence of Vpu at any of the concentrations.

While there has been controversy on the role of $\beta \mathrm{TrCP}$ in antagonism of BST2/tetherin by $\mathrm{Vpu}$, the studies refuting its importance have usually employed siRNA 
knockdown of the $\beta$ TrCPs, while this is to our knowledge the first time these proteins have been permanently knocked out in virus producing cells $(74,75)$. Vpu also has multiple mechanisms to counteract BST-2/tetherin, and so differing degrees of partial antagonism often remain when one of the mechanisms is blocked $(77,105)$. Even in the $\beta \operatorname{TrCP}$ knockout cells, there does remain a very slight degree of BST-2/tetherin antagonism, but it falls below statistical significance when BST-2/tetherin levels increase beyond 10ng. Thus, the $\beta$ TrCP-independent mechanism would seem to be most relevant when BST2/tetherin is expressed at low levels. In support of this, it was demonstrated previously that treatment of cells with MLN4924 to block Cullin ubiquitin ligases had greater impact on $\mathrm{Vpu}$ antagonism of BST-2/tetherin if cells were first treated with interferon, thus upregulating the BST-2/tetherin levels (81). In addition to BST-2/tetherin levels, the level of $\beta \mathrm{TrCP}$ expression may also affect the potency of Vpu activity against BST-2/tetherin. When we reintroduced $\beta$ TrCP-2 into cells where both genes had been knocked out the activity of Vpu against both CD4 and BST-2/tetherin was enhanced. Thus, the mechanism Vpu utilizes to counteract BST-2/tetherin likely varies depending on the levels of BST$2 /$ tetherin and of $\beta \operatorname{TrCP}$.

Finally, it should be noted that Vpu maintains at least a slight amount of activity against all three targets, (CD4, GaLV Env, and BST-2/tetherin) in the $\beta \mathrm{TrCP}-1 / 2 \mathrm{KO}$ cell line. It cannot be ruled out that this cell line maintained some $\beta \operatorname{TrCP}-2$ activity. The knockout cell line we generated targeted the third of seven of its WD40 domains that are required for interacting with its targets. Notably, we did not succeed in producing a homozygous knockout with a gRNA that targeted further upstream, and it has been reported that $\beta \operatorname{TrCP}-2$ (FBXW11) is essential for optimal proliferation (106). 
Author Contributions: Conceptualization, D.C., Y.E.S., T.M.L. and M.C.J. Methodology, D.C., Y.E.S., T.M.L., D.A.G, T.D.L. and M.C.J. Writing-Original Draft Preparation, D.C., Y.E.S., T.M.L. and M.C.J. Writing-Review and Editing, D.C., Y.E.S., T.M.L., D.A.G, T.D.L. and M.C.J. Funding Acquisition, M.C.J.

Acknowledgments: Research reported in the publication was supported by the National Institute of Allergy and Infectious Disease of the National Institutes of Health under award numbers R21AI131775 and R21AI112399. 


\section{Figure 2.1: Vpu depletes mature GaLV Env $\Delta 8$ in cells.}

(A) 293FT cells were transfected with hemagglutinin (HA)-tagged GaLV Env $\Delta 8$ and GFP-tagged Vpu. 16h post-transfection, cells were treated for $7 \mathrm{~h}$ with either a DMSO control, proteasomal inhibitor cocktail containing $10 \mathrm{uM}$ MG132 and $20 \mathrm{uM}$ epoximicin (EP), or a lysosomal inhibitor Bafilomycin A1 (BafA1, $100 \mathrm{nM})$. Then, 10ug of cell lysate was immunoblotted with anti-HA antibody. (B) 293FT cells were transfected with HIVCMV-GFP $\left(\mathrm{Vpu}^{+/}\right)$and GaLV Env $\Delta 8$ or GaLV Env $\Delta 12$. At $40 \mathrm{~h}$ post-transfection, cells were treated with either proteasomal inhibitors MG132 (10 uM) or ALLN (20 uM), or lysosomal inhibitors BafA $(100 \mathrm{nM})$ or Concanamycin A (ConA, $100 \mathrm{nM})$, a DMSO control, or no treatment (NT). After $12 \mathrm{~h}$, virus-containing culture media was collected and spinoculated onto $293 \mathrm{~T}$ mCAT-1. Transduced cells were measured by flow cytometry $48 \mathrm{~h}$ later. I.U.=infectious units. (A) was performed by Devon Gregory. (B) was performed by Tiffany Lucas. 
Figure 2.1: Vpu depletes mature GaLV Env $\Delta 8$ in cells.

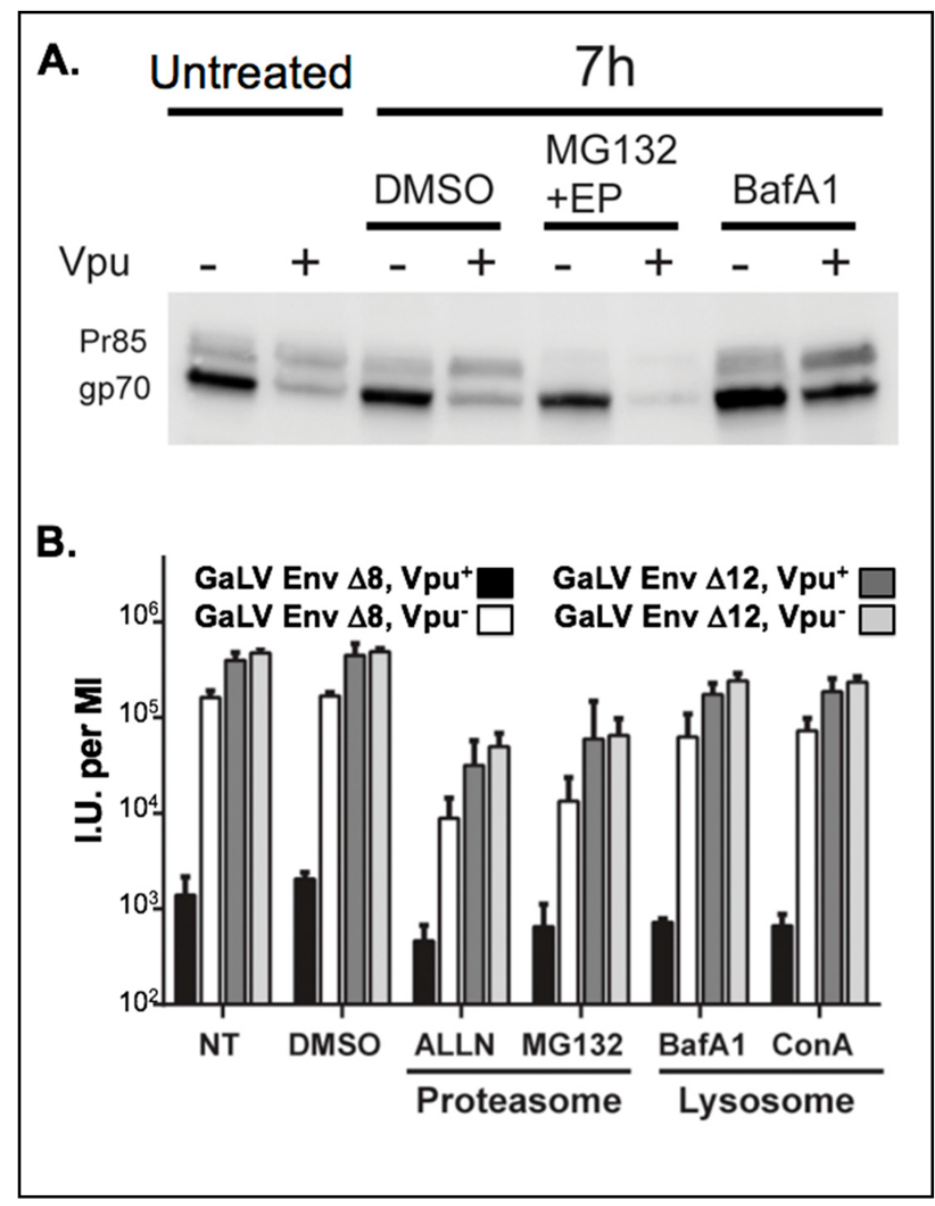




\section{Figure 2.2: Vpu decreases surface expression of GaLV Env $\Delta 8$ and infectious particle}

production.

(A) Sorted 293 TVA cells stably expressing mCherry tagged MLV GaLV Env $\Delta 8$ were transduced with HIV-CMV-NLS-GFP $\left(\mathrm{Vpu}^{+/}\right)$and plated on glass bottom dishes. The presence of GFP (green) indicates that the cell has been transduced. Total Env (cherry) expression is shown in red. Surface Env was labeled with Alexa405 (blue). Scale bar = 10uM. (B) Transduced cells from (A) were collected, surface labeled and analyzed on a flow cytometer for both total and surface MLV GaLV Env $\Delta 8$ expression. Transduced cells without Env stably expressed were used as controls (grey/yellow populations) (C) Average relative mean fluorescence intensity (MFI) of Env expression and surface Env expression are shown from 3 individual experiments. (D) Cells were transduced as explained in (A). Supernatants containing VLPs were collected from transduced Envexpressing producer cells $\left(\mathrm{Vpu}^{+/-}\right)$for infection on 293 mCAT-1. Producer cells were collected and analyzed by flow cytometry to determine the number of virus producing cells. Transduced mCAT-1 cells were measured by flow cytometry $48 \mathrm{~h}$ later and infectious unit production (I.U.) per million transduced producer cells was calculated. Figure 2.2 was performed by Yul Eum Song. 
Figure 2.2: Vpu decreases surface expression of GaLV Env $\Delta 8$ and infectious particle production.

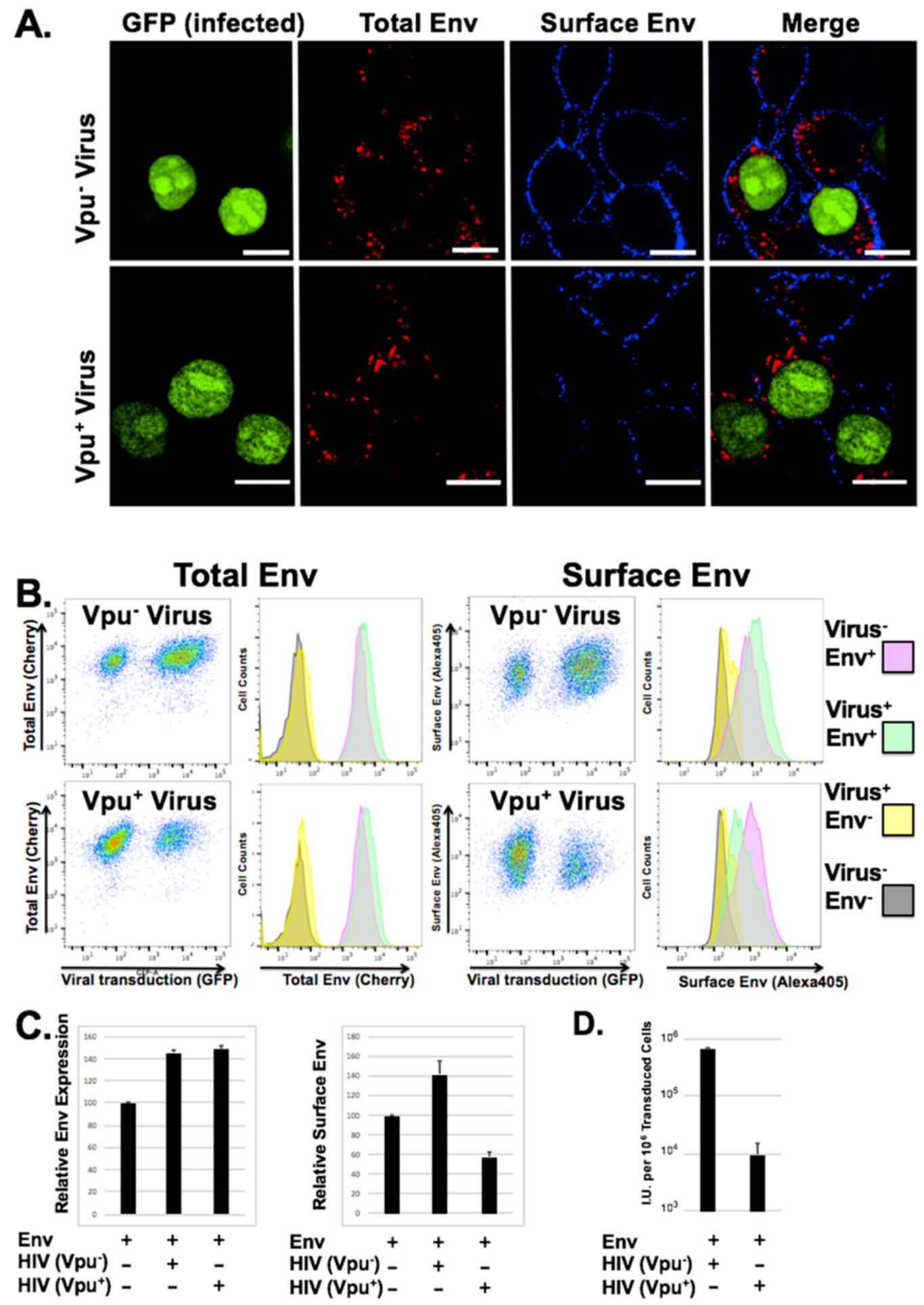


Figure 2.3: Vpu constrains GaLV Env envelope pseudotyping in a polyubiquitindependent manner.

293FT cells were transfected with Vpu sensitive GaLV Env $\Delta 8$ or insensitive GaLV Env $\Delta 12$, HIV-CMV-GFP and a DN ubiquitin (Ub-K0) expression construct. After 48h, media was transferred to $293 \mathrm{~T}$ mCAT-1 cells and infectivity was measured by flow cytometry $48 \mathrm{~h}$ later. Bars are mean $\pm \mathrm{SD}$ for three experimental replicates. Figure 2.3 was performed by Tiffany Lucas. 
Figure 2.3: Vpu constrains GaLV Env envelope pseudotyping in a polyubiquitindependent manner.

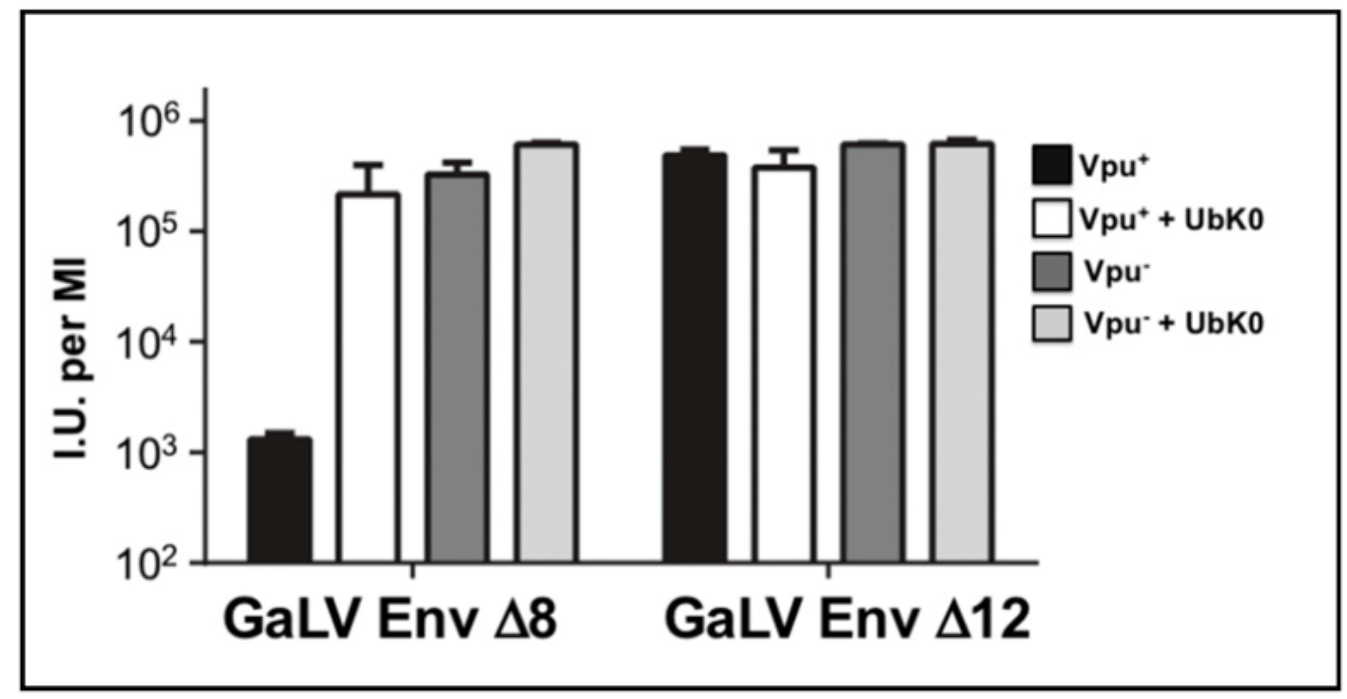




\section{Figure 2.4: Vpu antagonism of GaLV Env is Cullin1 dependent.}

(A) 293FT cells were transfected HIV-CMV-GFP along with either MLV Env or MLV/GaLV Env $\Delta 8$. 24h post-transfection cells were treated with $1 \mu \mathrm{M}$ MLN4924, or DMSO. Media was collected after $24 \mathrm{~h}$ and was transferred to $293 \mathrm{~T}$ mCAT-1 cells and infectivity was measured by flow cytometry $48 \mathrm{~h}$ later. Infectious particle output was normalized to that of HIV-CMV-GFP $\left(\mathrm{Vpu}^{-}\right) / \mathrm{DMSO}$. (B) 293FT cells were transfected with HIV-CMV-GFP along with either MLV Env or MLV/GaLV Env $\Delta 8$ and either DN Cullin1, DN $\beta$ TrCP, or empty vector control. After 48h, media was transferred to $293 \mathrm{~T}$ mCAT-1 cells and infectivity was measured by flow cytometry 48h later. (C) 293FT cells were transfected with human CD4 alone, CD4 + Vpu IRES GFP, or CD4 + Vpu IRES GFP

+ DN Cullin1 or DN $\beta$ TrCP. CD4 surface protein was labeled and analyzed by flow cytometry $48 \mathrm{~h}$ later. Asterisks indicate $\mathrm{p}<0.01$. (A) and (B) were performed by Daniel Cyburt. (C) was performed by Daniel Cyburt and Yul Eum Song. 
Figure 2.4: Vpu antagonism of GaLV Env is Cullin1 dependent.

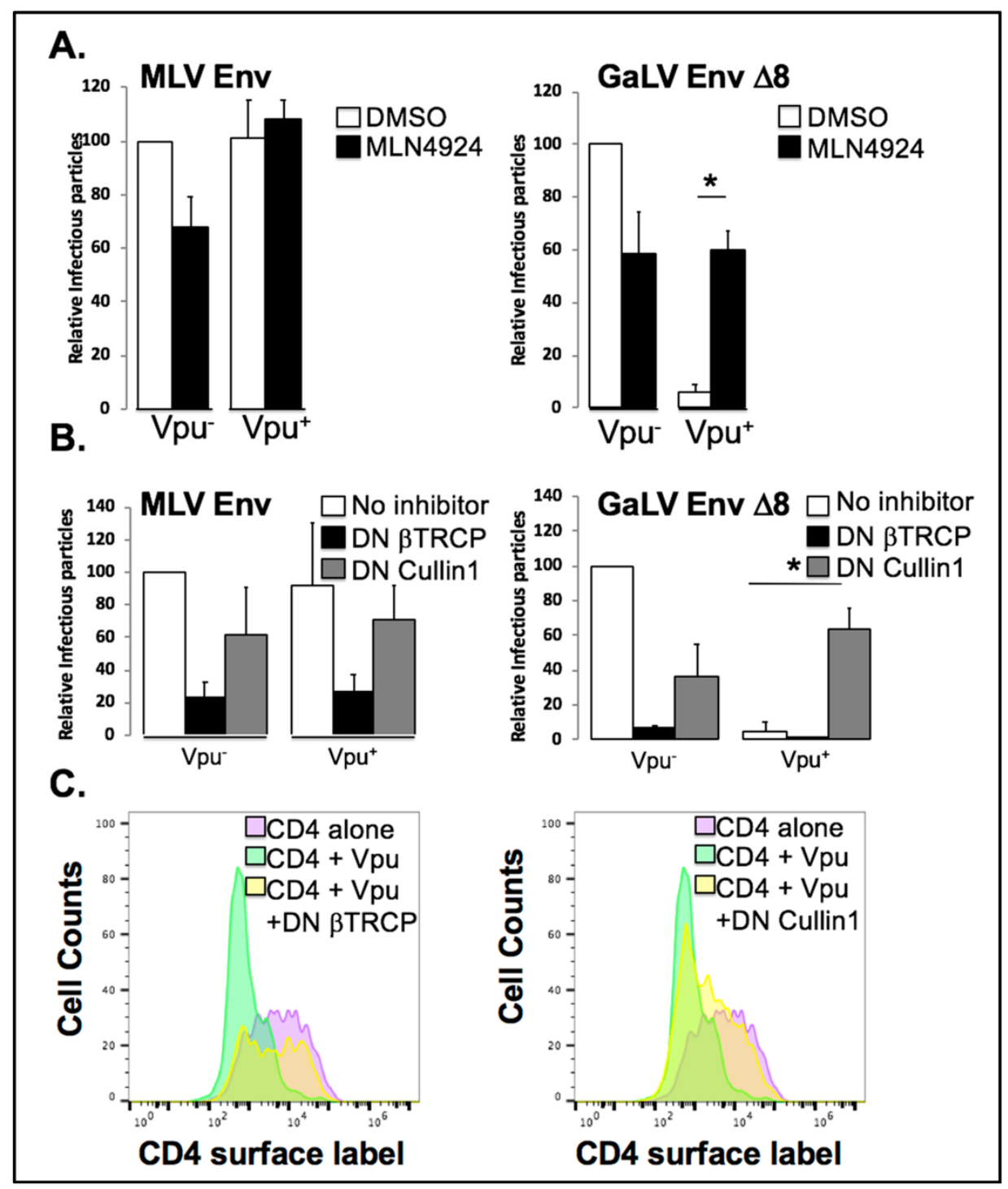




\section{Figure 2.5: Confirmation of $\beta \operatorname{TrCP}-1$ and $-2 \mathrm{KO}$.}

Shown is the schematic of the gRNA target site in the $\beta \operatorname{TrCP}-1$ and -2 genes. Genomic DNA was extracted from the parent 293FT cell line as well as the $\beta \operatorname{TrCP}-1 / 2$ KO cell line. The sequence flanking the CRISPR gRNA target sites was amplified by PCR and sequenced. The sequence in the $\beta \mathrm{TrCP}-1$ was a homogeneous $-2 \mathrm{nt} \mathrm{KO}$. The sequence in the $\beta \operatorname{TrCP}-2$ locus was appeared to be three distinct variations $(-2,-16,+62)$. This was confirmed by subcloning the PCR and sequencing the three distinct isoforms. Figure 2.5 was performed by Daniel Cyburt and Marc Johnson. 
Figure 2.5: Confirmation of $\beta \operatorname{TrCP} \mathbf{- 1}$ and $-2 \mathrm{KO}$.

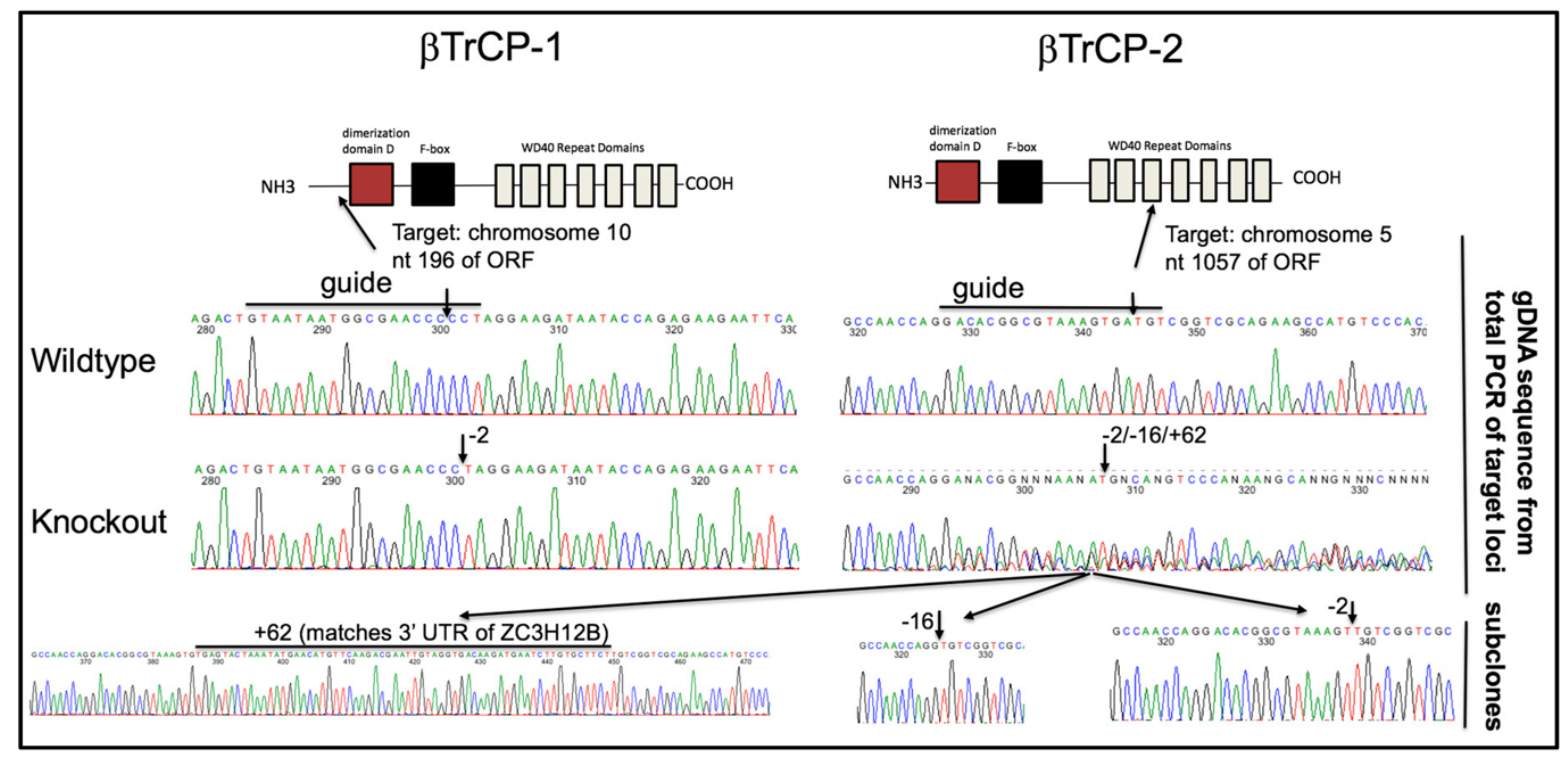




\section{Figure 2.6: Importance of $\beta \operatorname{TrCP}$.}

(A) 293FT, $\beta$ TrCP-1/2 KO, and add back cells were transfected HIV-CMV-GFP $\left(\mathrm{Vpu}^{+}, \mathrm{Vpu}^{-}\right.$, and $\left.\mathrm{Vpu}^{\mathrm{S} 52 / 56 \mathrm{~A}}\right)$ along with MLV Env or GaLV Env $\Delta 8$. After $48 \mathrm{~h}$, media was transferred to $293 \mathrm{~T}$ mCAT-1 cells and infectivity was measured by flow cytometry 48h later. Infectious particle production from each data set was normalized to HIV-CMVGFP ( $\left.\mathrm{Vpu}^{-}\right)$. Shown is the average and standard deviation from six independent experiments. (B) The same cell lines were transfected with human CD4 with or without Vpu-IRES-GFP, surface stained for CD4 expression, and analyzed by flow cytometer 48h later. (C) The same cell lines were transfected with HA-tagged human CD4 with or without Vpu-IRES-GFP, collected after 2 days, and immunoblotted for HA-CD4. GAPDH was blotted as a loading control. (D) The same cells lines were transfected with HIV-CMVGFP $\left(\mathrm{Vpu}^{+}, \mathrm{Vpu}^{-}\right.$, and $\left.\mathrm{Vpu}^{\mathrm{S} 52 / 56 \mathrm{~A}}\right)$, along with $\mathrm{VSV}-\mathrm{G}$ and varying amounts of HATetherin (0-80ng). After 48h, media was transferred to $293 \mathrm{~T}$ mCAT-1 cells and infectivity was measured by flow cytometry $48 \mathrm{~h}$ later. Infectious particle production from each data set was normalized to that of the well with $0 \mathrm{ng}$ tetherin. Shown is the average and standard deviation of seven independent experiments. Students t test was performed comparing $\mathrm{Vpu}^{+}$to $\mathrm{Vpu}^{\mathrm{S} 52,56 \mathrm{~A}}$. Asterisks indicate $\mathrm{p}<0.01$. NS indicates difference is not significant. (A) was performed by Daniel Cyburt. (B) was performed by Daniel Cyburt and Yul Eum Song. (C) was performed by Yul Eum Song. (D) was performed by Daniel Cyburt and Terri Lyddon. 
Figure 2.6: Importance of $\beta \operatorname{TrCP}$.

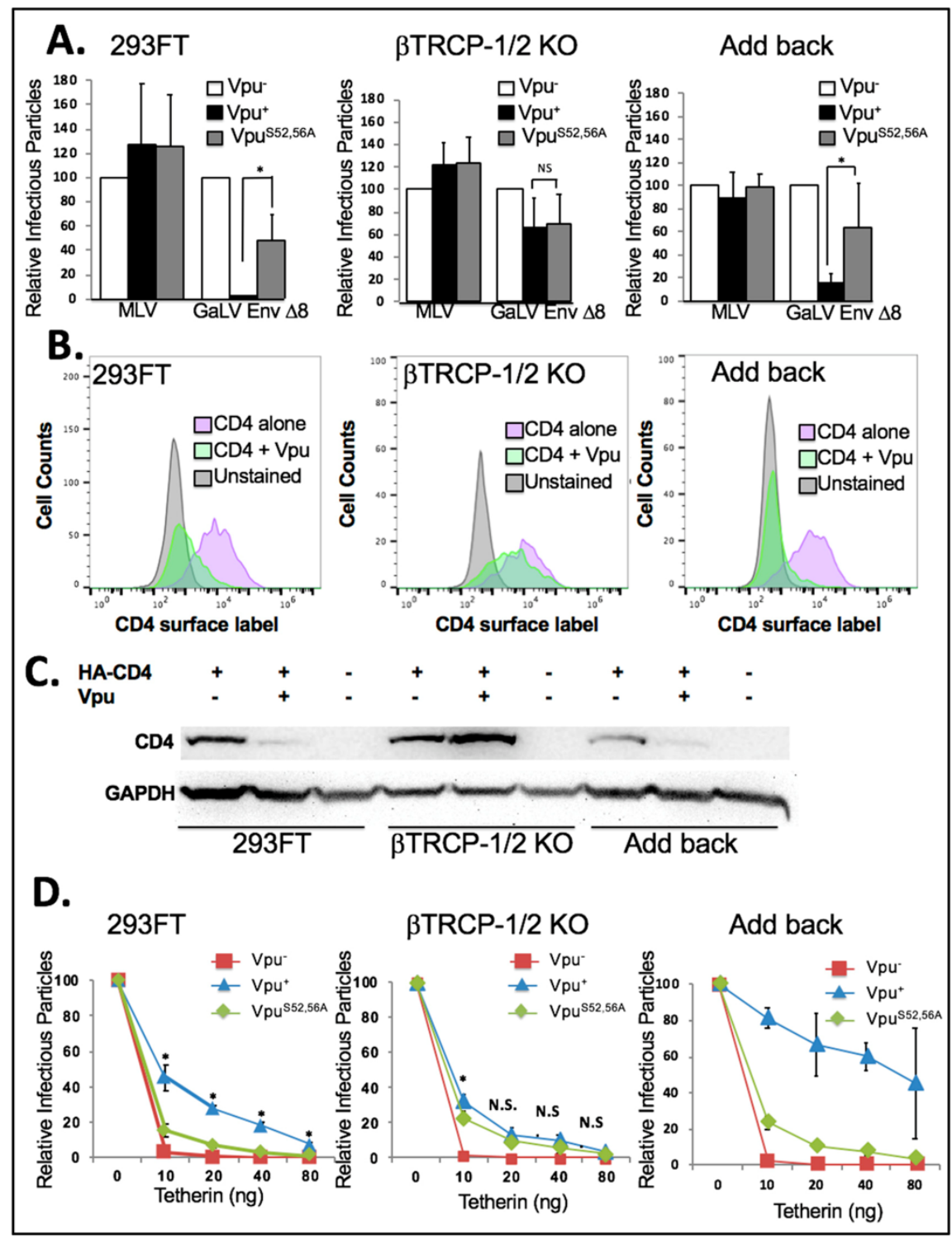




\section{Sequence determinants in gammaretroviral Env cytoplasmic tails dictate virus-specific pseudotyping compatibility}

Yul Eum Song, Grace Y. Olinger, Sanath Kumar Janaka, Marc C. Johnson

This work has been published in the Journal of Virology: Sequence determinants in gammaretroviral Env cytoplasmic tails dictate virus-specific pseudotyping compatibility. Journal of Virology 2019, doi: 10.1128/JVI.02172-18 (107).

\subsection{Abstract}

Viruses can incorporate foreign glycoproteins to form infectious particles through a process known as pseudotyping. However, not all glycoproteins are compatible with all viruses. Despite the fact that viral pseudotyping is widely used, what makes a virus/glycoprotein pair compatible is poorly understood. To study this, we chose to analyze a gammaretroviral glycoprotein (Env) whose compatibility with different viruses could be modulated through small changes in its cytoplasmic tail (CT). One form of this glycoprotein is compatible with Murine Leukemia Virus (MLV) particles but incompatible with Human Immunodeficiency Virus 1 (HIV-1) particles, while the second is compatible with HIV-1 particles but not with MLV particles. To decipher the factors affecting virusspecific Env incompatibility, we characterized Env incorporation, maturation, cell-to-cell fusogenicity, and virus-to-cell fusogenicity of each Env. The HIV-1 particle 
incompatibility correlated with less efficient cleavage of the R-peptide by HIV-1 protease. However, the MLV particle incompatibility was more nuanced. MLV incompatibility appeared to be caused by lack of incorporation into particles, yet incorporation could be restored by further truncating the CT or by using a chimeric MLV Gag containing HIV-1 MA, without fully restoring infectivity. The MLV particle incompatibility appeared to be caused in part by fusogenic repression in MLV particles through an unknown mechanism. This study demonstrates that the Env CT can dictate functionality of Env within particles in a virus-specific manner.

\subsection{Importance}

Viruses utilize viral glycoproteins to efficiently enter target cells during infection. How viruses acquire viral glycoproteins has been studied to understand the pathogenesis of viruses and develop safer and more efficient viral vectors for gene therapies. The CTs of viral glycoproteins have been shown to regulate various stages of the glycoprotein biogenesis, but a gap still remains in understanding the molecular mechanism of glycoprotein acquisition and functionality regarding the CT. Here we studied the mechanism of how specific mutations in the $\mathrm{CT}$ of a gammaretroviral envelope glycoprotein distinctly affect infectivity with two different viruses. Different mutations caused failure of glycoproteins to function in a virus-specific manner due to distinct fusion defects, suggesting that there are virus-specific characteristics affecting glycoprotein functionality. 


\subsection{Introduction}

An essential gene encoded by all retroviruses is Env, a transmembrane glycoprotein that mediates viral entry into target cells. Env is translated in the ER, transported to Golgi, and glycosylated and cleaved by a furin-type cellular protease into two subunits, SU (surface) and TM (transmembrane). The two subunits remain associated after cleavage as they traffic to the viral assembly site and are incorporated into viral particles. During entry of virus into the target cell, the SU subunit of Env first binds to the host cell receptor, triggering a conformational change, and the TM subunit subsequently facilitates fusion between the viral membrane and the host cell $(25,26,108)$.

Some retroviral Env proteins go through a priming step before becoming fusogenically active. For gammaretroviruses, the activated viral protease in the viral particle cleaves off a short segment of the Env CT, called the R-peptide, allowing Env to become fusogenic $(30,109,110)$. This cleavage affects trimeric interactions of TM and results in the splaying of the three TM legs, which is essential for receptor triggering (30). Although this type of Env activation has been most extensively studied in gammaretroviruses, the Env protein from the deltaretrovirus Mason-Pfizer monkey virus (MPMV), and the lentivirus Equine infectious anemia virus (EIAV), have also been reported to have an R-peptide (111-115). Viral maturation can also be involved in priming Env in other retroviruses; in the case of HIV-1, even though a specific R-peptide is not present, maturation affects Env conformation and fusogenicity in a CT dependent manner (116-118).

In addition to the direct regulation of fusion, the Env CT has also been reported to be involved in multiple roles in the viral life cycle including signaling, trafficking, incorporation, and interaction with host-factors $(38,119-122)$. Importantly, the Env CT of 
several proteins have been reported to affect their incorporation into foreign viral particles (112, 123-126). We reported previously that the CT of MLV Env is dispensable for recruitment to HIV assembly sites. However, we also found that MLV Env is selectively packaged into MLV particles over HIV particles if both are present in the same cell, and that this selective incorporation into MLV particles was CT dependent, suggesting specific interactions exist between the $\mathrm{CT}$ and the viral particle (42).

In this study, we characterized two distinct Env glycoproteins with changes in their CT that cause virus-specific incompatibility with either lentiviral (HIV-1) or gammaretroviral (MLV) particles. Our results demonstrate that mutations in the CT can have distinct effects on two different retroviruses, suggesting that the distinct environment within different types of retroviral particles can affect the functionality of Env.

\subsection{Materials and Methods}

Antibody generation. A humanized version of the rat IgM monoclonal antibody 42/114 was generated for detection of the MLV TM (p15E) protein. To accomplish this, RNA from the 42/114 hybridoma (127) was extracted, cDNA was generated, and the sequence for the variable regions of $\mu$ heavy chain (Forward primer GAGGTGCAGCTTCAGGAGTCAGGACCTGGCCTT, reverse primer ATCAGACAGGGGGCTCTCGCAGGAGA) and $\kappa$ light chain (Forward primer TTAGGGCTGCTGCTGCTTTGG，reverse primer CGTTTCAGTTCCAGCTTGGT) were PCR amplified. The extracted variable regions were subcloned by in-fusion (clontech)

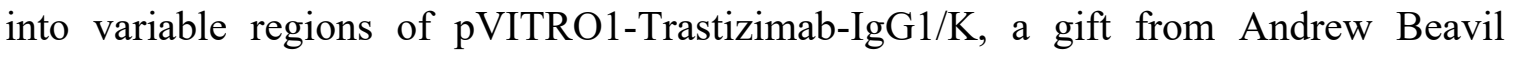
(Addgene pasmid \#61883) (128). Complete $\gamma$ heavy chain and $\kappa$ light chain from the vector 
were then PCR amplified and inserted into retroviral vectors pQCXIP and pQCXIH, respectively (clontech) and used to generate a stable 293FT cell line secreting human IgG. Collected antibodies secreted in medium were further purified with HiTrap Protein G column (GE Health Science), dialyzed, and concentrated by filter centrifugation for 45-90 minutes at 4,000 rpm (Amicon Ultra 15).

Cell culture. The 293FT cell line was obtained from Invitrogen. The 293T mCAT-1 cell line expressing the ecotropic F-MLV Env receptor was kindly provided by Walter Mothes (Yale University). The HT1080/mCAT-1 Cre reporter cell line was kindly provided by Alan Rein (20). 293T mCAT-1 cells stably expressing Gaussia luciferase downstream of a Tet Responsive Element (TRE) promoter was previously described (129). All cells were maintained in DMEM supplemented with 10\% fetal bovine serum, $2 \mathrm{mM} \mathrm{L-glutamine,} 1$ $\mathrm{mM}$ sodium pyruvate, $10 \mathrm{mM}$ non-essential amino acids, and 1\% MEM vitamins.

Plasmids. NL4-3 derived HIV-CMV-GFP was kindly provided by Vineet Kewal-Ramani (National Cancer Institute [NCI]-Frederick). This proviral vector lacks Vif, Vpr, Vpu, Nef, and Env, and contains CMV-GFP in place of Nef. CMV-MLV-GagPol and the ecotropic Friend MLV Env expression vector were kindly provided by Walter Mothes (Yale University). The MLV reporter constructs pQCXIP-TdTomato and pQCXIP-GFP were previously described $(51,130)$. The plasmid encoding Cre recombinase fused with MLV Gag (MLV Gag-Cre) was kindly provided by Alan Rein (20). For Src-MLV GagPol, the Src variant (MGSNKSKPKDASSQR) was cloned in the place of MA in CMV-MLVGagPol. CMV driven tTA (tet-off) protein expression vector was previously described (129). The HIV-1 vector with CMV-driven, intron-interrupted reverse Gaussia luciferase 
(HIV-iGluc) was adapted from a vector kindly provided by David Derse and was described previously $(129,131-133)$. The MLV reporter vector with a CMV-driven, reverse-introninterrupted Gaussia luciferase gene (MLV-iGluc) was constructed by subcloning the CMV-driven reverse-intron-interrupted Gaussia luciferase gene from the HIV-iGluc into pQCXIP (Clontech). The MLV GagPol construct with HIV-1 MA domain (hMA-MLV) was previously described (134). The MLV Env with GaLV Env CT was previously described (51). MLV/GaLV Env $\Delta 8$ construct and MLV/GaLV Env I170P $\Delta 8$ (I26P in previous report) constructs were previously described (56). Point mutations and truncations of MLV and MLV/GaLV Env were created by oligonucleotide-mediated mutagenesis. Some Env constructs used in the western blots contain a hemagglutinin (HA) tag (YPYDVPDYA) in a proline-rich region in SU (129). For virus capture assay, codon optimized MLV/GaLV $\Delta 8$ Env with internal HA tag in the proline-rich region in SU (Addgene) was inserted in pQCXIP (Clontech), and the HA tag was replaced with GFP. The VSV-G expression construct was obtained from NIH AIDS Reagent Program (135). For the RSV proviral construct, CMV was inserted upstream of the GFP gene in the previously described RCAS.GFP-Tomato vector (136). pUC19 was obtained from Invitrogen.

Infectivity assay. Infectivity assays using HIV-CMV-GFP or CMV-MLV-GagPol and pQCXIP-GFP with various Env constructs were performed by transfection in 293FT cells with polyethylenimine (PEI) (137). Media were changed about 24 hours post-transfection and collected after another 24 hours. Supernatant containing the virus was frozen at $-80^{\circ} \mathrm{C}$ for at least 4 hours, thawed and spun at 3,200 xg for 5 minutes, and the same volume of media was added on target cells with $20 \mu \mathrm{g}$ of hexadimethrine bromide per mL (Sigma 
H9268). Infected cells were collected about 48 hours post-infection, fixed with $4 \%$ paraformaldehyde, washed with PBS, and analyzed on an Accuri C6 flow cytometer.

Except where noted, transfections for the infectivity assay were performed in 6well plates with a total of $2 \mu \mathrm{g}$ of plasmids. For HIV-1 infectivity, 1,800 ng of HIV-CMVGFP and 200 ng of Env constructs were co-transfected. For MLV infectivity, 900 ng of CMV-MLV-GagPol and $900 \mathrm{ng}$ of either pQCXIP-GFP or pQCXIP-TdTomato were transfected along with $200 \mathrm{ng}$ of Env. For the Env titration assay, $400 \mathrm{ng}$ of HIV-CMVGFP or $400 \mathrm{ng}$ of CMV-MLV GagPol and $400 \mathrm{ng}$ of pQCXIP-TdTomato were transfected with various amounts of Env (20 ng, $80 \mathrm{ng}, 320 \mathrm{ng}, 1280 \mathrm{ng}$ ) and a filler plasmid, pUC19, was used to normalize total DNA quantity in the transfection. For western blotting and infectivity in figures 3.6C, $8 \mathrm{C}$ and 9 , a total of $1 \mu \mathrm{g}$ of plasmid was transfected at a 9 to 1 ratio of virus to Env. For western blot and infectivity in figures 3.4, and 5C, 1,500 ng of virus and $500 \mathrm{ng}$ of Env were transfected.

Virus capture assay. The virus capture assay was performed using a modified version of our published protocol $(129,131)$. Briefly, 293FT cells in 12 well plates were transfected with HIV-iGluc (400 ng) or MLV-iGluc (200 ng) and MLV-CMV-GagPol (200 ng) along with various GFP-tagged Env expression plasmids (40 ng for HIV-1 particles, $50 \mathrm{ng}$ for MLV particles) and the VSV-G expression plasmid (10 ng for HIV-1, $25 \mathrm{ng}$ for MLV). These amounts were derived empirically from titration curves to yield similar infectivity between the two different virus constructs. Medium was changed after 24 hours and collected after another 24 hours. $20 \mu \mathrm{l}$ (or $100 \mu \mathrm{l}$ for Env truncations) of PBS with or without polyclonal GFP antibodies previously described $(1: 10,000,(129))$ was added to the wells of an ELISA plate and incubated overnight at $4^{\circ} \mathrm{C}$. The antibody or PBS was then 
removed and replaced with blocking buffer (PBS with 1\% BSA, 5\% sucrose, $0.05 \%$ sodium azide) for 1 hour. After blocking buffer was removed, 10ul of viral supernatant and 10ul of PBS was added to the each well and incubated for 3 hours at $37^{\circ} \mathrm{C}$. After the incubation, supernatant was removed and the wells were washed twice with $100 \mu 1$ of PBS, except the positive control wells where the viral supernatant was not removed. $20 \mu \mathrm{l}$ of PBS was then added to washed wells to normalize the volume, and 293FT cells were added on top for infection. 2 days later, $20 \mu \mathrm{l}$ of supernatant from each well was transferred to a black 96 well plate for measuring guassia luciferase activity with $50 \mu \mathrm{l}$ of $10 \mu \mathrm{M}$ coelenterazine in $0.1 \mathrm{M}$ Tris ( $\mathrm{pH} 7.4)$ and $0.3 \mathrm{M}$ sodium ascorbate (NanoLight Technology). Luminescence, representing infectivity, was measured from the supernatant using a PerkinElmer Enspire 2300 Multilabel Reader. Each sample was normalized to the straight infection (no wash) as positive controls.

Viral entry assay. The viral entry assay was performed as previously described (20), with a few modifications. Briefly, 293FT cells in 12 well plates were transfected with $50 \mathrm{ng}$ of each Env expression plasmid, $225 \mathrm{ng}$ of CMV-MLV-GagPol, $225 \mathrm{ng}$ of pQXIP-GFP, and 45 ng of MLV Gag-Cre fusion plasmid. In some instances these transfections were performed in 6 well plates with appropriate scaling of plasmids. After 24 hours of transfection, medium was changed, and the supernatant was harvested after another 24 hours. Media were frozen at $-80^{\circ} \mathrm{C}$ for at least 4 hours, thawed, and Cre reporter cells were transduced with equal volumes of the virus samples. After 48 hours, firefly luciferase activity was measured using Bright Glo reagent (Promega). 
Env fusion assay. 293FT cells were transfected with $100 \mathrm{ng}$ of Env and $100 \mathrm{ng}$ of tTA expression plasmid in a 6-well plate or half this amount was transfected in 12 wells. The next day, medium was changed to remove residual transfection reagent, and 293 mCAT-1 TRE Gluc cells were plated on top of the transfected 293FT cells. After an additional 24 hours, $20 \mu \mathrm{l}$ of supernatant from co-cultured cells was assayed for luminescence in triplicate as previously mentioned.

Western blotting. Virus samples were concentrated by centrifugation at $30,000 \mathrm{x} g$ for 2 hours through a $20 \%$ sucrose cushion. $1 \mathrm{~mL}$ of media were used for probing $\mathrm{SU}$ and $1.5 \mathrm{ml}$ of media were used to probe for $\mathrm{p} 15 \mathrm{E}$. For probing $\mathrm{SU}$ of $169 \mathrm{~F}^{*}, 170 \mathrm{I}^{*}$, and $170 \mathrm{P}^{*}$ constructs, $0.6 \mathrm{ml}$ of media were used. Viral pellets were resuspended and denatured by heating in sample buffer (50mM Tris, 2\% sodium dodecyl sulfate [SDS], 20\% glycerol, 5\% -mercaptoethanol) before loading. For probing SU, a 10\% PAGE gel and 0.45um PVDF for transfer were used. For probing p15E, a 15\% PAGE gel and 0.22um PVDF for transfer were used. In both experiments, membranes were blocked with $2 \%$ nonfat dry milk prior to antibody incubation. For detecting SU, goat anti-MLV Env gp70 (kindly provided by Alan Rein, NCI-Frederick) diluted 1:10,000 was used. HA-tagged SU Env was detected using anti-HA antibody diluted in 1:1,000 (Sigma, H3663). TM p15E antibody was generated in human IgG form and $6 \mu \mathrm{g}$ of purified antibody was used per $1 \mathrm{ml}$ of PBS-T without milk. Anti-HIV p24 hybridoma medium was used at 1:500 (HIV-1 p24 hybridoma [183-H12-5C], obtained from NIH AIDS Reagent Program) from Bruce Chesebro (138) and anti-MLV capsid (CA) medium from hybridoma (R187, ATCC) (139) was used at 1:250. After washing with PBS, HRP-conjugated secondary antibody was used at 1:10,000 for all blots. Horseradish peroxidase-linked anti-mouse (A5278), anti-goat (A8919), anti- 
rat (A5795), anti-human (A0293) were obtained from Sigma. Luminata Classico Western HRP substrate (Millipore) was used for visualization of the membranes with a chemiluminescence image analyzer Fuji Film LAS-3000 or UVP BioSpectrum 815 Imaging System.

\subsection{Results}

Mutations in the GaLV Env CT dictate virus-specific compatibility. We and others have previously demonstrated that GaLV Env is incompatible with HIV-1, and that this incompatibility is dictated by the CT. This incompatibility can be imparted onto MLV Env by replacing the MLV Env CT with the GaLV Env CT (MLV/GaLV Env, Figure 3.1A). The incompatibility of the GaLV Env CT was found to be largely dependent on the HIV1 accessory protein Vpu $(50,51)$. However, even in the absence of Vpu, HIV-1 infectivity with MLV/GaLV Env is reduced by more than 10-fold compared to MLV Env (Figure 3.1B). This incompatibility of MLV/GaLV Env with HIV-1 is relieved by deletion of 8 amino acids from the C-terminus of the $\mathrm{CT}(\mathrm{MLV} / \mathrm{GaLV} \Delta 8)$.

We showed previously that the CT of MLV Env can dictate selective packaging of MLV Env into MLV particles (42). While exploring the sequences in the CT that dictate selective packaging into MLV particles, we serendipitously found that an additional point mutation into $\mathrm{MLV} / \mathrm{GaLV} \Delta 8$, I170P (referred to as I26P in the previous report, nomenclature defined by $170^{\text {th }}$ residue of MLV TM), resulted in a glycoprotein that was compatible with HIV-1 particles but remarkably less compatible ( 100-fold) with MLV particles (Figure 3.1B and 1C) (56). Henceforth this construct will be referred to as I170P $\Delta 8$. Because these few differences in MLV/GaLV Env and I170P $\Delta 8$ present opposite 
phenotypes in virus-specific compatibility, we sought to identify the factors dictating these incompatibilities.

Vpu independent HIV-1 particle incompatibility motifs in GaLV Env CT reside on 171N, 178K, and 179I. MLV/GaLV (170-196) Env consistently shows a 10-fold or greater drop in infectivity with HIV-1 particles compared to the parent MLV Env. To narrow down the precise sequence responsible for the incompatibility, several additional chimeras of Env were generated with varying amounts of GaLV Env CT sequence (Figure 3.2A). Interestingly, when most of the MLV Env CT was exchanged with GaLV sequence (176-196, which includes the protease cleavage site), the chimeric Env remained more compatible than the MLV/GaLV (170-196) Env with HIV-1 particles (Figure 3.2B). However, when the chimeric MLV/GaLV (170-186) Env was generated, the infectivity dropped to a level similar to MLV/GaLV (170-196) Env, suggesting that the region between 170-186 dictates the incompatibility. Among the 17 amino acids in this region, seven positions differ between the MLV and GaLV Env CTs (Figure 3.2C). We created point mutations between these residues to determine the precise residues in the GaLV Env CT that dictate HIV-1 particle incompatibility (Figure 3.2D). Starting from the MLV Env CT backbone, which is compatible with HIV-1 particles, only one of the substitutions to GaLV Env sequence, A179I, caused a great reduction in infectivity with HIV-1 particles, though this mutation also reduced infectivity with MLV particles. This suggests that more than one amino acid change to MLV Env would be required to enforce virus-specific incompatibility. Starting from the GaLV Env CT, which is not compatible with HIV-1 particles, three point mutations, N171K, K178Q, and I179A, alleviated the incompatibility with HIV-1 particles (Figure 3.2D). I170V in the GaLV Env backbone showed somewhat 
moderate recovery of HIV-1 particle infectivity, although this mutation still showed a statistically significant difference $(\mathrm{p}<0.01)$. Introduction of the GaLV Env amino acids [N171, K178 and I179] or [I170, N171, K178, and I179] (subsequently referred to as MLV Env NKI and INKI, respectively) into the MLV Env CT was sufficient to make MLV Env incompatible with HIV-1 particles (Figure 3.2E). These chimeras retain compatibility with MLV particles.

\section{Env NKI incompatibility with HIV-1 particle is related to Env maturation,} while I170P Env incompatibility with MLV particle is related to Env incorporation. Next, we sought to investigate the mechanism behind virus-specific Env incompatibility of MLV Env NKI and I170P $\Delta 8$. First, the effects of increasing amounts of Env in transfections were tested to determine whether the incompatibility is due to Env expression level (Figure 3.3). Viral infectivity with an incompatible Env was not restored with either Env at higher concentrations, suggesting the incompatibility is not related to Env expression levels.

We also examined whether the Env incompatibility was due to loss of Env incorporation. Viruses with each Env were produced and concentrated to conduct western blotting (Figure 3.4A). Incorporation of I170P $\Delta 8$ into MLV particles was significantly lower than in HIV-1 particles, suggesting the incompatibility is at least in part due to an incorporation block. MLV Env NKI was incorporated into HIV-1 particles slightly less efficiently than into MLV particles, though this reduction did not seem to correlate with the large loss of infectivity.

Next, we sought to examine whether the incompatibility of incorporated MLV Env NKI is due to a defect in Env activation during virus maturation. To test if the 
incompatibility is due to the lack of R-peptide cleavage, the same virus from above was also blotted for the Env TM subunit (Figure 3.4A). As it is shown, MLV/GaLV $\Delta 8$ was cleaved efficiently in both HIV-1 and MLV particles, while MLV Env NKI was only partially cleaved in HIV-1 particles and at least half of the TM domain remained in the non-cleaved Pr15E form, suggesting the MLV Env NKI incompatibility with HIV-1 particles is due to inefficient cleavage, hence a fusogenic defect. I170P $\Delta 8$ was found to be properly cleaved in HIV-1 and MLV particles, though very little was present in MLV particles.

Another approach, viral capture, was also used to verify loss of I170P $\Delta 8$ incorporation. Previously we found that different Env glycoproteins can be co-packaged into the same viral particles (131). Taking advantage of this co-packaging, virus can be captured with an antibody to one glycoprotein, and then the infectivity, driven by the second glycoprotein, can be measured. Thus, the infectivity can be used as a readout for incorporation of the captured glycoprotein into particles. The advantage of this assay is that the signal can only come from incorporation into actual viral particles and not any other forms of glycoprotein shedding. Viral particles were produced with GFP-tagged Envs and VSV-G. The particles were captured by GFP antibody and the infectivity was measured by co-packaged VSV-G (Figure 3.4B). In this experiment, MLV/GaLV Env and I170P $\Delta 8$ were incorporated equally into HIV-1 particles, but I170P $\Delta 8$ was incorporated at least 10 times less than MLV/GaLV Env into MLV particles.

\section{The I170P $\Delta 8$ incorporation defect in MLV particles is alleviated by CT truncations}

or replacing MLV MA. Since the I170P residue is located on the N-terminal portion of the CT, we sought to investigate whether the C-terminus of the Env CT contributes to the 
incompatibility. Additional C-terminal truncations of MLV/GaLV Env and I170P Env were generated (Figure 3.5A). As shown in Figure 3.5B, these truncations partially restore the infectivity of Envs with the I170P mutation with MLV particles, but even the truncation with the highest infectivity (I170P $\Delta 25$ ) was still down over 10-fold compared to MLV/GaLV $\Delta 25$. To investigate whether the Env incorporation correlated with the infectivity, western blotting was conducted (Figure 3.5C). Despite the poor recovery in infectious particle production, these truncations led to efficient Env incorporation into MLV particles. For example, the 16 amino acid truncation restored nearly wildtype incorporation levels, despite their infectivity remaining down almost 100 -fold. This phenotype was recapitulated with the virus capture assay (Data not shown). Each of the truncations was also tested with HIV-1 particles. Incorporation into HIV-1 particles was relatively constant among all of the Envs. Surprisingly, smaller truncations to MLV/GaLV Env $(\Delta 12, \Delta 16, \Delta 20)$ enhanced the infectivity 3-7 fold with HIV-1 particles, but the same truncations in Env proteins with the I170P mutation did not enhance infectivity (Figure $3.5 \mathrm{~B}$ and $5 \mathrm{C})$.

If direct interactions exist between Gag and the Env CT, these interactions would most likely involve the membrane binding MA domain of Gag. Indeed, several mutations in HIV MA have been shown to affect Env incorporation, and in one case a mutation in HIV MA was even shown to affect R-peptide cleavage of MLV Env (140-148). To test if MA contributes to the I170P incompatibility, the MA domain of MLV Gag was replaced with HIV-1 MA (hMA-MLV) or the short membrane binding domain from Src (Src-MLV, Figure 3.6A). Both of the chimeric proteins were able to incorporate MLV/GaLV Env and form infectious particles (Figure $3.6 \mathrm{~B}$ and $6 \mathrm{D}$ ). With $\mathrm{I170 \textrm {P }} \Delta 8$, incorporation was enhanced with both chimeras relative to wildtype MLV Gag, but the infectivity with the 
Gag chimeras was only modestly increased with I170P $\Delta 8$ and remained down $\sim 8$-fold or more relative to $\mathrm{MLV} / \mathrm{GaLV} \Delta 8$. To determine if inefficient R-peptide cleavage was occurring, we probed the TM domain and found that it was efficiently cleaved with all three constructs. As a further evidence that the I170P defect is not related to maturation, truncation of the R-peptide (I170P $\Delta 16$ ) did not restore the infectivity of hMA-MLV (Figure 3.6C). These data suggest that the MA domain plays a role in the Env incorporation, but there are additional factors that contribute to the virus-specific functionality of the Env.

\section{I170P Env retains fusogenicity out of virions but not within the MLV particles.}

Because the various Env proteins display different levels of functionality depending on the type of viral particles they are incorporated into, we next sought to test the functionality of the Env proteins when expressed alone. To do this, Env constructs were transfected along with the transcriptional activator tTA (Tet-off) in 293FT cells, and the transfected cells were co-cultured with cells stably expressing the MLV Env receptor, mCAT-1, and a TREdriven gaussia luciferase. When the Env on the transfected cell surface fuses with the mCAT-1 receptor on the neighboring cell, tTA is transmitted to the reporter cells and turns on the TRE-induced gaussia luciferase gene. Luciferase activity was measured and normalized to constitutively active MLV Env $\Delta 26$ (Figure 3.7A). Both MLV/GaLV $\Delta 8$ and I170P $\Delta 8$ show low signal, as expected, since both Envs contain a portion of the Rpeptide. MLV/GaLV $\Delta 20$ and I170P $\Delta 20$ were chosen to test the fusogenicity since both Envs lacked an R-peptide and were incorporated at close to wildtype levels, yet I170P $\Delta 20$ displayed more than 100-fold lower infectivity with MLV particles compared to MLV/GaLV $\Delta 20$ (Figure 3.5). As expected MLV/GaLV $\Delta 20$ displayed greatly enhanced 
fusogenicity compared to MLV/GaLV $\Delta 8$. Fusogenicity of I170P $\Delta 20$ was slightly lower than $\mathrm{MLV} / \mathrm{GaLV} \Delta 20$, though the difference was not statistically significant.

Next, we proceeded to investigate the fusogenicity of I170P Env proteins within MLV particles. To test this, we used a system developed by Ahi et al (20) which utilized MLV Gag fused with Cre recombinase and an HT1080/mCAT-1 Cre reporter cell line. When the Env binds the mCAT-1 receptor and mediates fusion, cleaved Cre recombinase enters the cell and induces recombination that turns on the luciferase gene. Luciferase signal was measured and normalized to MLV Env. As it is shown, I170P Envs $\Delta 8, \Delta 12$, $\Delta 16$, and $\Delta 20$ all displayed at least 25 -fold less fusogenicity from within MLV particles than MLV/GaLV Env counterparts (Figure 3.7B). Further truncation of the I170P Env CT $(\Delta 24, \Delta 25)$ increased fusogenicity within particles up to about half of the MLV/GaLV Env counterparts. As before, truncation of I170P Env enhanced incorporation into viral particles, but incorporation did not correlate with fusogenicity. Most notably, I170P $\Delta 20$ had essentially wildtype incorporation, but its fusogenicity was still reduced 25 -fold.

\section{Hydrophobicity is important at the $170^{\text {th }}$ residue of the GaLV Env CT. Several reports} suggest that retroviral Env CTs contain amphipathic helixes including the lytic peptides in the long CT of lentiviral Envs $(38,121)$. Gammaretroviral Env CTs also have conserved amphipathic regions with proposed alpha helical structure (56, 149-153). Among the amphipathic sequences present on the gammaretroviral Env CT, hydrophobic residues appear every three or four amino acids, supporting the possibility of the helix having a hydrophobic face. This helix has been proposed to affect membrane curvature during fusion (152). Because the $170^{\text {th }}$ residue on MLV and GaLV Env would be predicted to be part of this hydrophobic face, we hypothesized that the hydrophobicity of the $I 170^{\text {th }}$ residue 
is critical in Env fusogenicity in MLV particles and introduced mutations to that residue to test it (Figure 3.8A). I170P $\Delta 20$ was chosen for introducing point mutations because it retains MLV incompatibility, is constitutively active, and is incorporated into MLV particles at near wildtype levels. Among the Envs with a mutation at the $170^{\text {th }}$ residue tested, a mutation leading to an alternative hydrophobic residue, leucine, exhibited wildtype infectivity with both HIV-1 and MLV particles (Figure 3.8B). Mutations that introduced charged residues, glutamic acid and lysine, showed low infectivity, with a 25 -fold reduction in infectious HIV-1 particle production and a 100-fold or greater reduction in infectious MLV particle production, which is an even greater effect than that observed with the proline mutation. Introduction of glycine and serine residues exhibited intermediate phenotypes. With these mutations, infectivity dropped about 2-fold in HIV-1 particles while the infectivity was down about 15-30 fold in MLV particles. These results suggest that hydrophobicity is functionally important at this position, supporting the importance of the proposed alpha helical structure of CT. The original proline mutation (I170P) exhibits about 75 -fold reduction in MLV infectivity, which could be due to both the reduced hydrophobicity and the disruption of the helix structure in the CT. The hydrophobic $170^{\text {th }}$ residue is very well conserved in other gammaretroviruses, further suggesting the importance of this residue (Figure 3.8A). For all the mutations tested, HIV-1 particles were shown to be generally more tolerant to changes than MLV particles.

To test if the incompatibility is specific to MLV particles, next we investigated whether the I170P Env retains incompatibility in a different retrovirus family, Rous sarcoma virus (RSV, strain RCAS) (Figure 3.8C). RSV particle infectivity with I170P $\Delta 20$ was also greatly reduced compared MLV/GaLV $\Delta 20$, and the reduction was similar to that seen with MLV particles. 
The $170^{\text {th }}$ residue in GaLV Env CT is critical for MLV infectivity. Truncation analysis (Figure 3.5) showed that the mutation I170P affected MLV particle compatibility even if the protein was truncated immediately after that residue. Next, we analyzed the effects of truncating Env at position $169\left(169 \mathrm{~F}^{*}\right)$, eliminating the position all together. This truncated Env was efficiently incorporated into MLV and HIV-1 particles, but the functionality was considerably lower in MLV particles than in HIV-1 particles (Figure 3.9). Adding the $170^{\text {th }}$ residue enhanced functionality with MLV particles by over 4-fold if the $170^{\text {th }}$ residue was an Isoleucine $\left(170 I^{*}\right)$, but did not enhance it at all if it was a proline (170P*). All three of the glycoproteins were similarly functional with HIV-1 particles. These data suggest that the 1170 residue of Env is critical for functionality with MLV particles, but not with HIV-1 particles. In a previous study we showed that large truncations in MLV Env affected functionality with MLV particles more than with HIV-1 particles (42), but we were surprised to find here that the loss of functionality was not due to reduced incorporation. These data suggest that Env functionality is modulated in a virusspecific manner through an unknown mechanism.

\subsection{Discussion}

Viral Env glycoprotein acquisition has been studied not only to understand the biological mechanisms of viral assembly and pathogenesis but also to enhance the current knowledge on more efficient and safe application of gene therapy. Even though various studies address Env incompatibility in retroviral vectors, a gap remains in understanding the mechanism of Env recruitment and incorporation especially regarding the CT. 
In this study, we investigated the mechanism behind virus-specific incompatibility dependent on the Env CT. The GaLV Env CT incompatibility with HIV-1 has been extensively studied and we and others reported that the incompatibility was partly dependent on Vpu $(50,51,58,112,123,154)$. Here we address the GaLV Env CT incompatibility remaining in the absence of Vpu. Interestingly from the exchanged segments of the CT between MLV and GaLV, we found that residues 170-186 of GaLV Env recapitulated the incompatibility with HIV-1 particles, but residues 176-196 of GaLV Env did not (Figure 3.2). This finding initially led us to believe that the incompatibility was not due to a cleavage defect because the R-peptide cleavage site-containing region (176-186) was identical in the two chimeras. This was surprising because a previous study had suggested that the GaLV Env R-peptide is poorly cleaved in HIV-1 particles (50). However, because the MLV Env with NKI residues from 170-186 region of GaLV displayed a cleavage defect, it became clear that amino acids upstream of the cleavage site also influenced cleavage efficiency. Among the three residues, two were at the cleavage site (Q178K, A179I) and one was just upstream of the cleavage site (K171N). This trio of changes modestly reduced Env incorporation, but noticeably reduced R-peptide cleavage and infectivity with HIV-1 particles.

For the MLV particle-specific incompatibility, MLV/GaLV I170P $\Delta 8$ Env was shown to be functional with HIV particles, but not with MLV particles. This particular mutant gained our attention because the defect was both striking and virus-specific. Introduction of the I170P mutation to MLV/GaLV $\Delta 8$ dropped the infectivity of MLV particles by approximately 100-fold, but had essentially no effect on the infectivity of HIV1 particles. Our analyses have revealed that this stark apparent difference in virus-specific incompatibility was in fact due to three different factors. 
The first factor causing incompatibility of Env proteins containing the I170P mutation with MLV particles is lack of incorporation. While incorporation of Env proteins into HIV-1 particles is not affected by the I170P mutation, the incorporation into MLV particles is severely reduced. We postulate that the loss of incorporation is because the I170P mutation changes the overall structure of the CT making it unable to fit into the lattice formed by the MLV MA domain. Similarly, the MA domain of HIV-1 Gag has been shown to affect HIV-1 Env incorporation, and compensatory mutations near the MA trimer interface rescue Env mutants with incorporation defects $(145,146,148)$. Consistent with our postulate, we found that the incorporation defect of I170P Env in MLV particles was alleviated by replacing MLV MA with HIV-1 MA or the Src-membrane binding domain (Figure 3.6). In addition, truncation of the Env CT progressively restored incorporation into MLV particles (Figure 3.5). However, the infectivity of MLV particles in both of these cases was only partially restored, suggesting that other factors contribute to the I170P defect in MLV particles.

The second factor contributing to the apparent virus-specific defect was the observation that the I170P mutation appears to have opposing positive and negative effects on infectivity with HIV-1 particles. Because the introduction of I170P into MLV/GaLV $\Delta 8$ Env had no effect on HIV-1 infectivity, we first interpreted this to mean that the mutation did not alter the functionality of the protein. However, further truncations of wildtype $\mathrm{MLV} / \mathrm{GaLV} \operatorname{Env}(\Delta 12, \Delta 16, \Delta 20)$ were found to noticeably enhance the infectivity of HIV-1 particles (3-7 fold), but these same truncations had no effect on HIV1 particle infectivity in the presence of the I170P mutation. We interpret this to mean that there is something in the R-peptide region that suppresses HIV-1 infectivity, but is alleviated by the I170P mutation. The most likely explanation is that there is a difference 
in the efficiency of R-peptide cleavage as was observed with other full length GaLV CT constructs (Figure 3.4). Although we did not observe a difference in R-peptide cleavage in HIV-1 particles, it is possible that such a difference was too small to be apparent, or that other factors such as the kinetics of R-peptide cleavage contributed to the effect. Nonetheless, the introduction of I170P into MLV/GaLV Env did have a negative impact on HIV-1 particle infectivity, but this impact was only obvious if the Env had most or all of its R-peptide deleted $(\Delta 12, \Delta 16, \Delta 20$, Figure 3.5). However, this 5-8 fold reduction in infectivity with HIV-1 particles was still noticeably less than the 50-fold or greater reduction seen with MLV particles, suggesting that the incompatibility is still partially virus-specific.

The third factor contributing to the apparent virus-specific defect was the virusspecific compatibility of I170P Env proteins in particles. Even when incorporation levels were equivalent and the R-peptide was removed, MLV/GaLV Env proteins containing I170P were less functional in MLV particles than in HIV-1 particles (Figure 3.5). We postulate that this difference in the two types of particles is due to differences in the lipid membrane that interacts with the Env CT. The CTs of MLV and GaLV Env are thought to form amphipathic helixes $(56,149,150)$, and a series of hydrophobic amino acids including I170 are highly conserved and would form one face of that helix. The amphipathic membrane-proximal region in the cytoplasmic tail of MLV Env was also previously reported to be critical for Env fusogenecity and hypothesized to affect membrane curvature during the fusion $(152,153)$. In our study, in the context of MLV/GaLV $\Delta 20$, mutation of the $170^{\text {th }}$ position to any non-hydrophobic amino acid greatly reduced infectivity, although the effect was always more pronounced in MLV particles than in HIV-1 particles (Figure 3.8). We reported previously that large truncations 
in the CT of MLV Env had a greater effect on MLV infectivity than on HIV-1 infectivity (42), but we were surprised to find here that the effect was not due to differences in Env incorporation. An Env protein truncated to one residue before I170 (169F*) was over 4fold less functional in MLV particles than an Env truncated immediately after I1 70 (170I*), yet the two Env proteins were equally infectious in HIV-1 particles, and the two Env proteins were equally incorporated into both HIV-1 and MLV particles. We propose that this amino acid interacts with the viral membrane to aid in fusion activity, and that minor differences between the HIV-1 and MLV membranes make the position more critical in MLV particles. If this model is correct, it is surprising that substitution of MLV MA with HIV-1 MA does not alleviate the necessity of this residue in MLV particles. However, because MLV Gag contains the p12 domain between MA and CA, the MA domain of HIV1 Gag would not necessarily be in the same configuration in MLV particles as in HIV-1 particles, and thus not necessarily interact with the membrane in the same way.

Overall, we show two different mutations in the CT of Env that lead to virusspecific incompatibility in distinct ways. While GaLV Env incompatibility with HIV-1 is largely related to insufficient R-peptide cleavage, I170P Env incompatibility with MLV is due to a mixture of factors. The $170^{\text {th }}$ residue in the Env TM is critical in MLV particles, but not in HIV-1 particles. How these mutations in the GaLV cytoplasmic tail affect virusspecific fusion remains a matter of speculation.

Acknowledgements. We thank Alan Rein for providing the materials for the MLV virusto-cell fusion assay. The following reagents were obtained through the NIH AIDS Reagent Program, Division of AIDS, NIAID, NIH: pHEF-VSVG from Dr. Lung-Ji Chang (Cat\# 
4693), Anti-HIV-1 p24 Hybridoma (183-H12-5C) (Cat\# 1513) from Dr. Bruce Chesebro. This work was supported by National Institute of General Medicine under award R01GM110776. 
Figure 3.1: Mutations in the Env cytoplasmic tail cause virus-specific incompatibility.

(A) Schematic of Env cytoplasmic tail variants. Number follows from N-terminus of MLV Env TM. (B) Relative percent infectivity with different Env variants normalized to MLV Env. Average and standard deviation from three independent experiments is shown. (C) Relative percent infectivity with various mutated Env proteins normalized to MLV Env. Number follows from N-terminus of MLV Env TM. Average and standard deviation from three independent experiments is shown. $*^{*}=\mathrm{p}<0.01$; $^{*}=\mathrm{p}<0.05$ by unpaired and two-tailed Student's $t$ test for each Env. 
Figure 3.1: Mutations in the Env cytoplasmic tail cause virus-specific incompatibility.
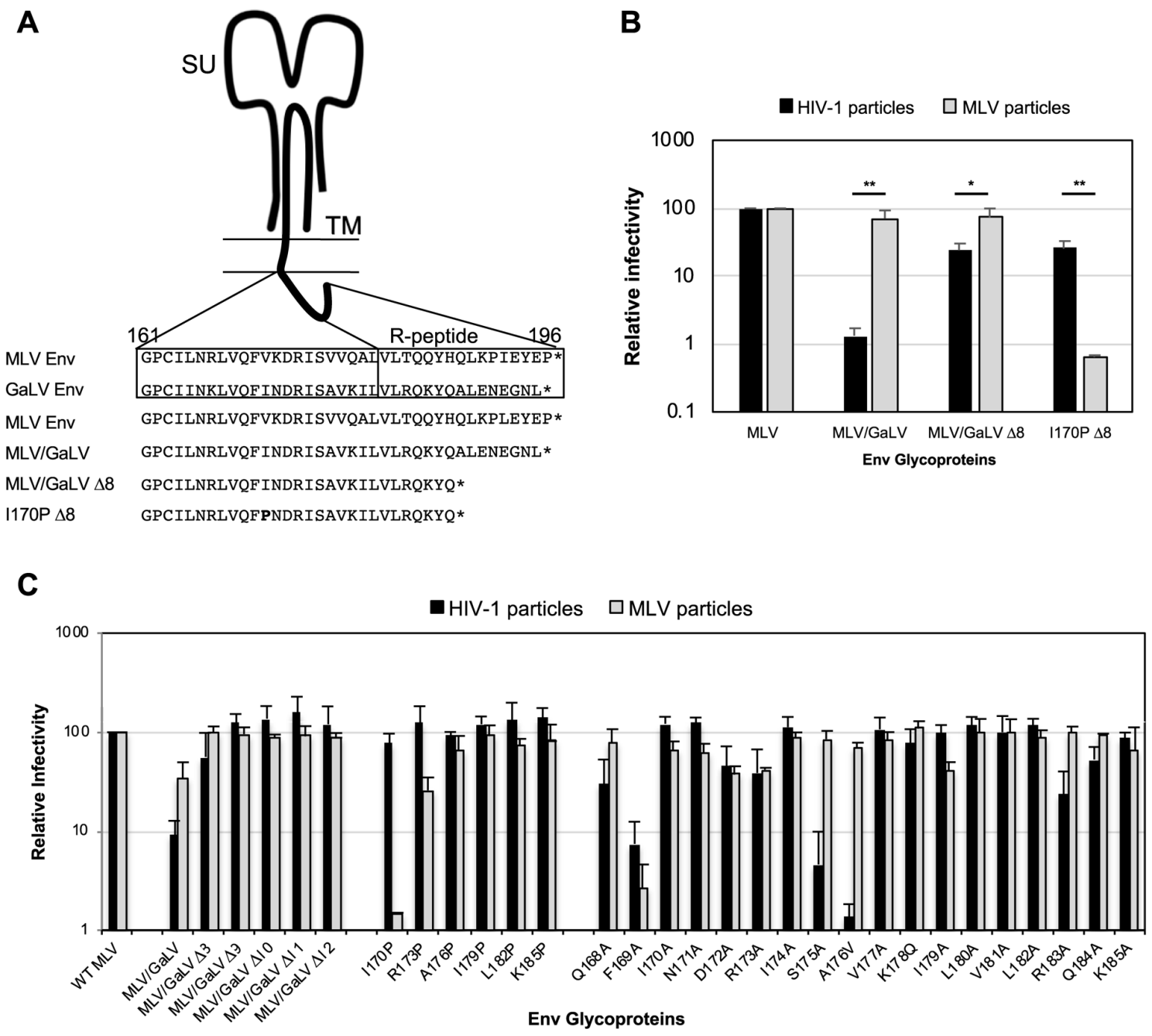
Figure 3.2: GaLV incompatibility with HIV-1 particles is modulated by residues $171 \mathrm{~N}$, 178K, and 179I.

(A) Amino acid sequences of MLV and GaLV Env cytoplasmic tail. Chimeric mutations are indicated with bolded font. (B) Relative percent infectivity with different chimeric Env proteins. (C) Comparison of amino acid sequences between MLV and GaLV in 170-186 region. Variable amino acids are indicated with grey font. (D) Relative percent infectivity of Env point mutants. (E) Relative percent infectivity of MLV Env combination mutants. MLV Env with K171N, Q178K, A179I mutations and V170I additional mutations are indicated as MLV Env NKI and MLV Env INKI. Infectivity is normalized to MLV Env. Average and standard deviation from three independent experiments is shown. $* * *=$ $\mathrm{p}<0.001 ; * *=\mathrm{p}<0.01 ; *=\mathrm{p}<0.05 ; \mathrm{ns}=$ not significant by unpaired and two-tailed Student's $t$ test for each Env. 
Figure 3.2: GaLV incompatibility with HIV-1 particles is modulated by residues $171 \mathrm{~N}$, 178K, and 179I.

\section{A} MLV Env MLV/GaLV (170-196) MLV/GaLV (190-196) MLV/GaLV (187-196) MLV/GaLV (176-196) MLV/GaLV (170-186) MLV INKI MLV NKI GPCILNRLVQFVKDRISVVQALVLTQQYHQLKPLEYEP GPCILNRLVQF INDRISAVKILVLRQKYQALENEGNL GPCILNRLVQFVKDRISVVQALVLTQQYHQLENEGNL GPCILNRLVQFVKDRISVVQALVLTQQYQALENEGNL GPCILNRLVQFVKDRISAVKILVLRQKYQALENEGNL GPCILNRLVQFINDRISAVKILVLROKYHQLKPLEYEP GPCILNRLVQF INDRISVVKILVLTQQYHQLKPLEYEP GPCILNRLVQFVNDRISVVKILVLTQQYHQLKPLEYEP
C

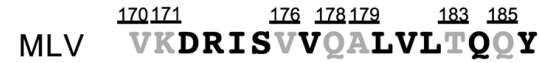
GaLV INDRISAVKILVLRQKY
B

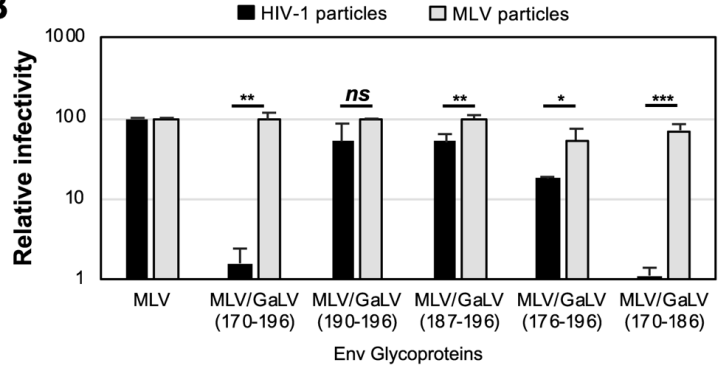

E

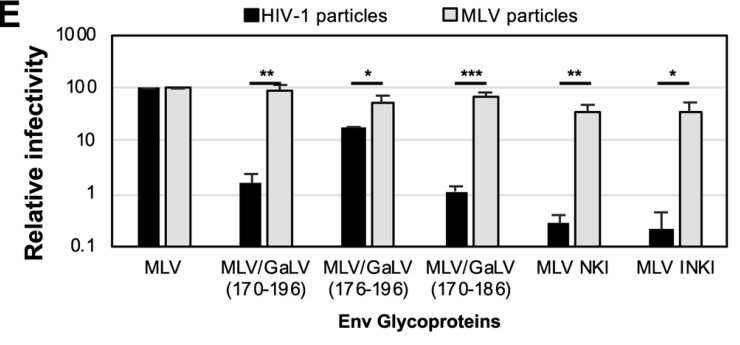

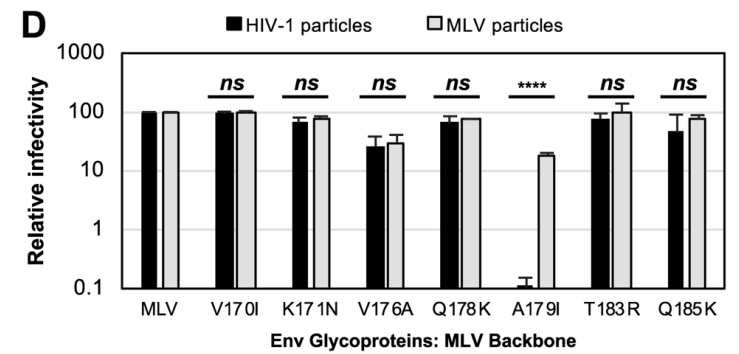

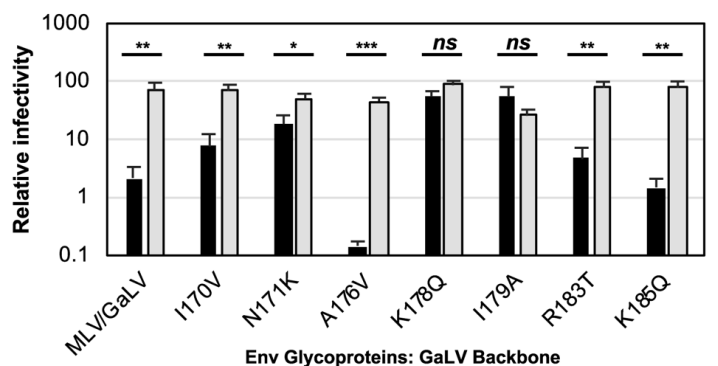


Figure 3.3: Env incompatibility is expression level independent.

Relative percent infectivity with increasing amounts of Env (20 ng, 80 ng, 320 ng, $1280 \mathrm{ng}$ ), normalized to $20 \mathrm{ng}$ of MLV Env is shown. Average and standard deviation from three independent experiments is shown. 
Figure 3.3: Env incompatibility is expression level independent.

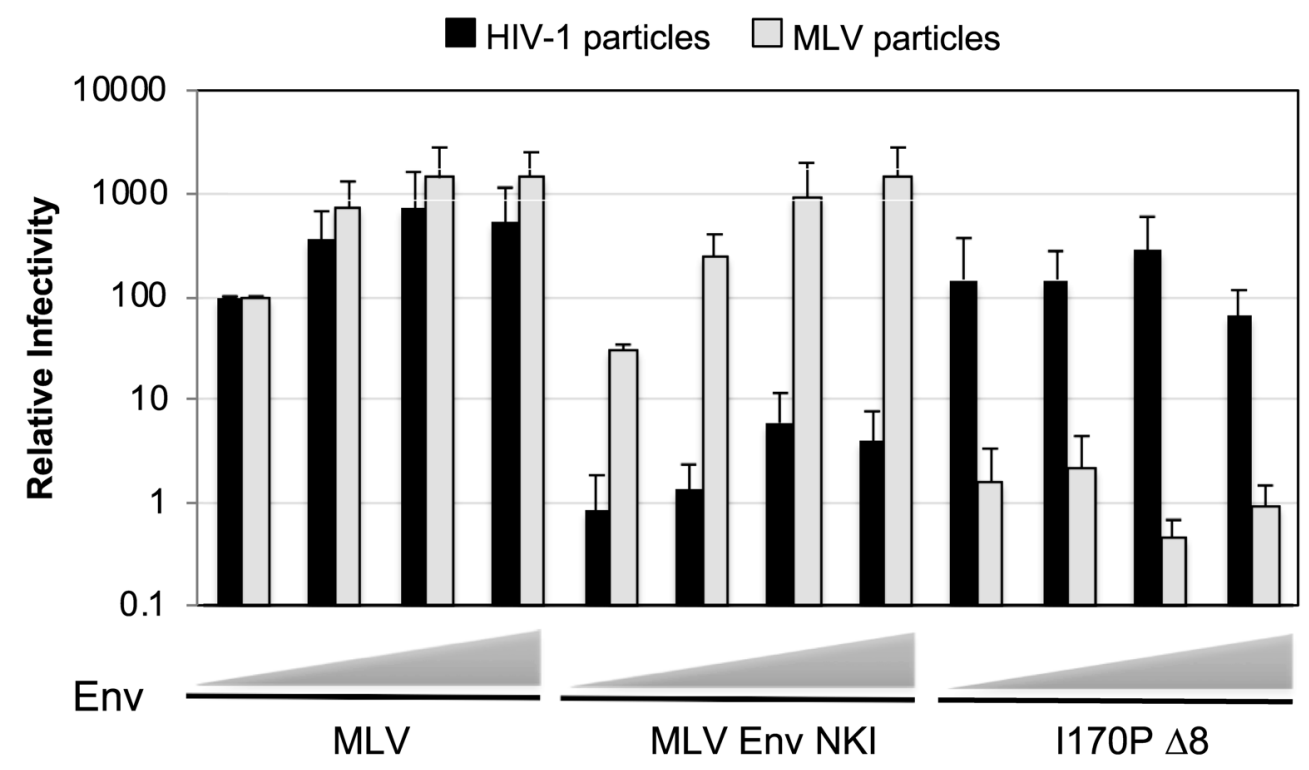


Figure 3.4: I170P Env is incorporation defective in MLV particles, but MLV Env NKI is maturation defective in HIV particles.

(A) Western blotting was performed with purified virus on SU (HA), p15E TM, and CA. Relative percent infectivity normalized to MLV/GaLV $\Delta 8$ is shown from the same viral media used for the western blot. Shown is a representative example of three independent experiments. (B) Virus capture assay with HIV-1 and MLV iGluc vectors and GFP-tagged Envs. Viruses in the collected supernatant was captured with GFP-antibody coated on ELISA plates, washed, infected with 293FT cells with co-captured VSV-G, and Gaussia luciferase activity was measured. Env incorporation was presented by signal from infected cells with captured virus. Each sample was normalized to positive controls (straight infection without wash). Average and standard deviation from 3 independent experiments is shown. 
Figure 3.4: I170P Env is incorporation defective in MLV particles, but MLV Env NKI is maturation defective in HIV particles.

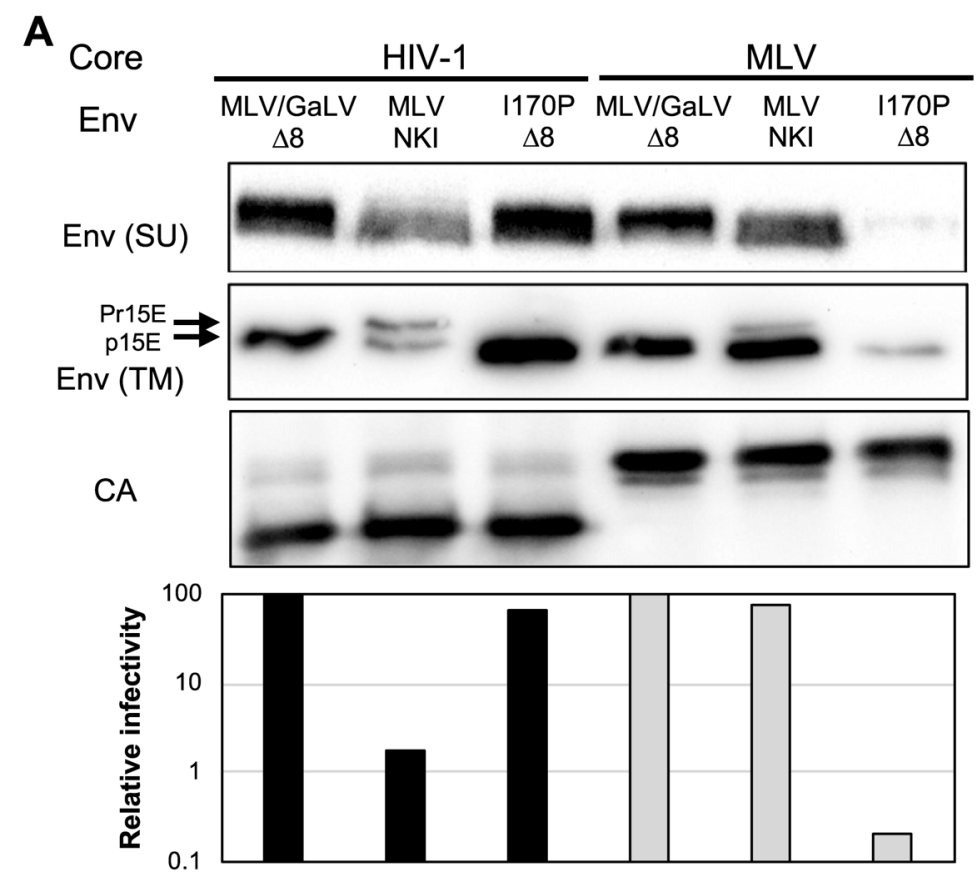

B

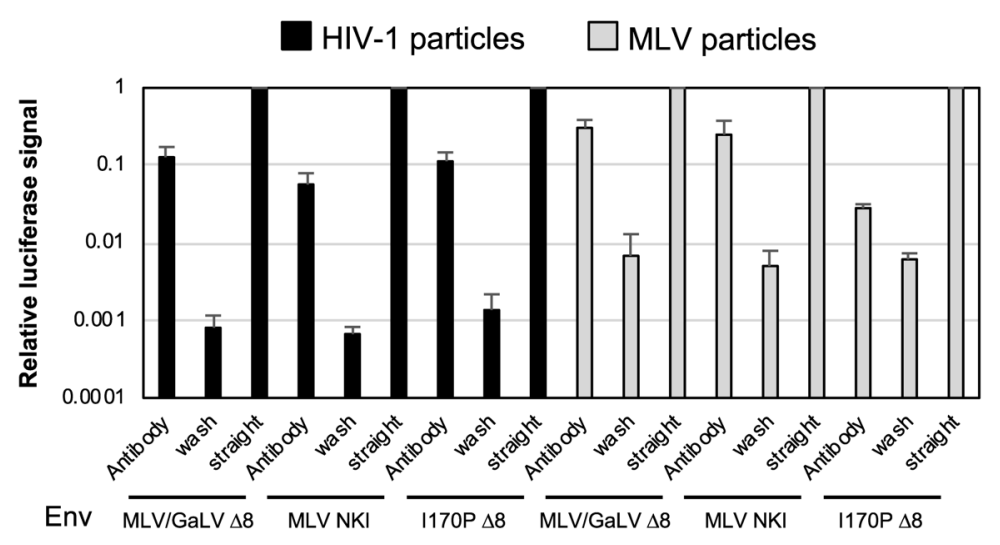


Figure 3.5: I170P Env incompatibility is partially alleviated by truncation of the Env CT.

(A) Schematic diagram with amino acid sequences with Env truncations. (B) Relative percent infectivity normalized to $\mathrm{MLV} / \mathrm{GaLV} \Delta 8$. Average and standard deviation from three independent experiments for each is shown. (C) Western blot of purified HIV-1 and MLV particles with denoted Env shows Env incorporation and capsid release. Relative percent infectivity of the media used in western, normalized to MLV/GaLV $\Delta 8$ is shown. Shown is a representative example of two independent experiments. 
Figure 3.5: I170P Env incompatibility is partially alleviated by truncation of the Env

CT.
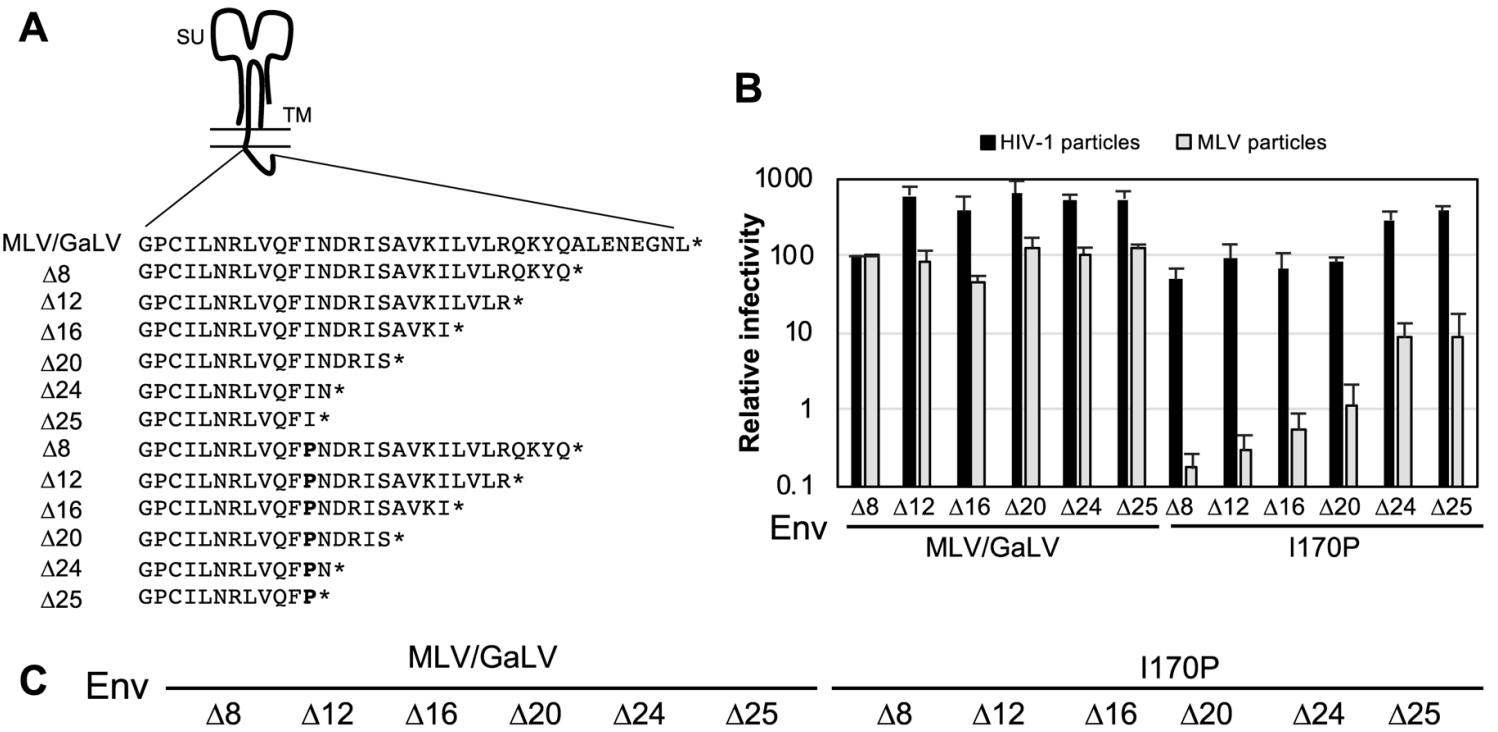

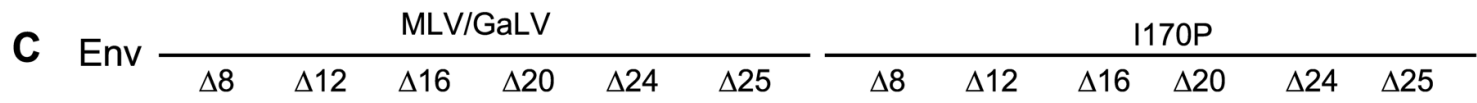

MLV particles

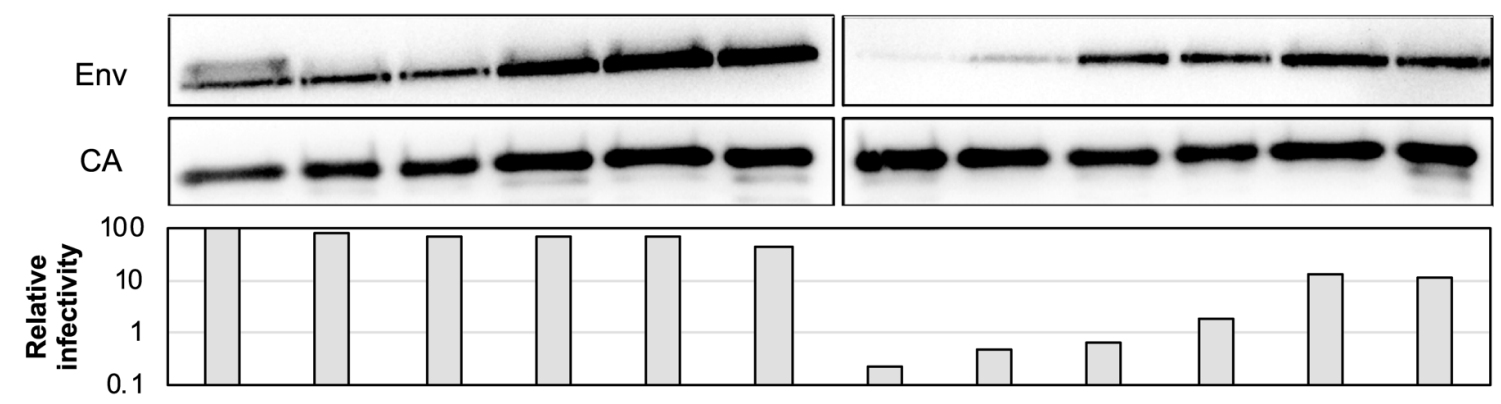

HIV-1 particles

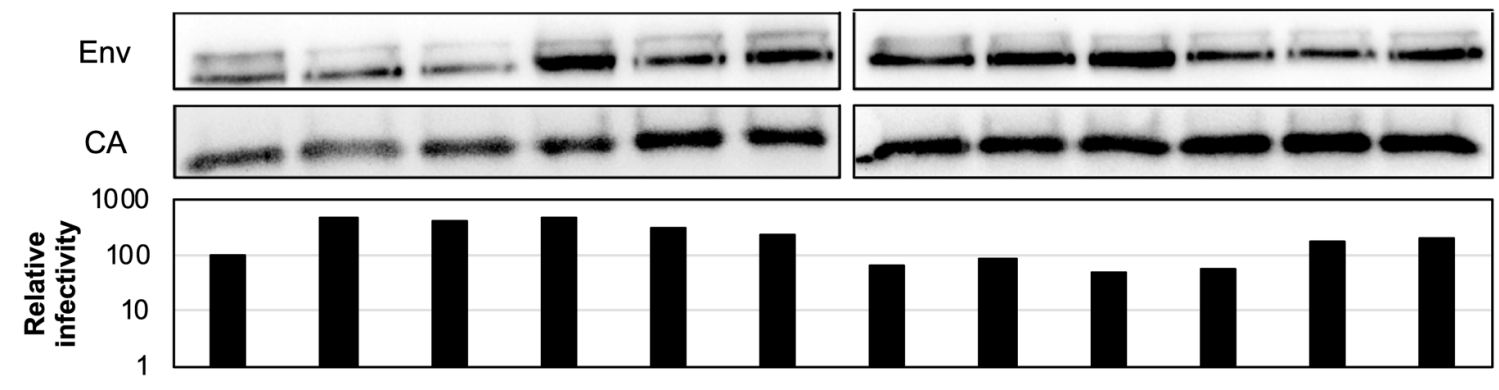




\section{Figure 3.6: I170P Env incompatibility partially alleviated by replacing MLV MA.}

(A) Schematic diagram of chimeric MLV Gag constructs used. (B) Western blot of purified HIV-1 and MLV particles with denoted Env shows Env incorporation and capsid release. Average and standard deviation from 6 independent experiments including infectivity from media used for western blot is shown. Western blot image is a representative example of two independent experiments. (C) Relative percent infectivity normalized to HIV-1 with MLV/GaLV $\Delta 16$. Average and standard deviation from three independent experiments is shown. (D) Virus capture assay with MLV, hMA-MLV, SrcMLV Gag with iGluc vectors and GFP-tagged Envs. Env incorporation was presented by signal from infected cells with captured virus. Each sample was normalized to positive controls (straight infection without wash). Average and standard deviation from 3 independent experiments is shown. 
Figure 3.6: I170P Env incompatibility partially alleviated by replacing MLV MA.

A

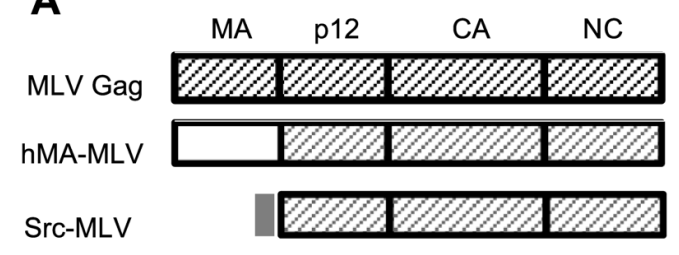

C

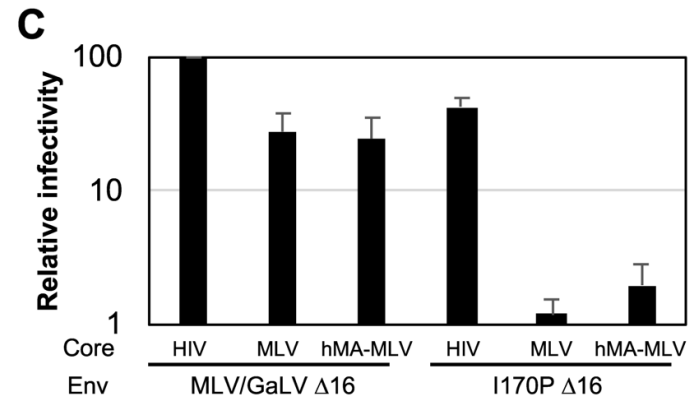

B

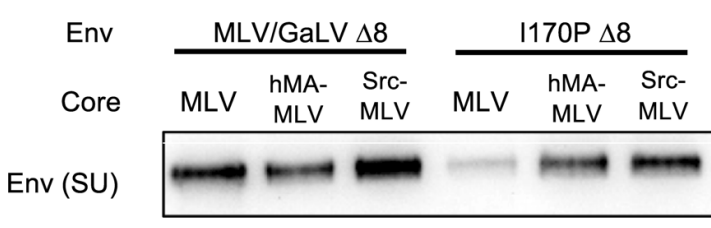

Env (TM)

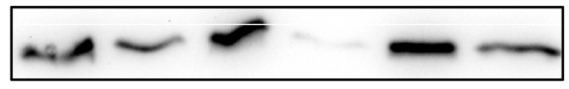

MLV CA
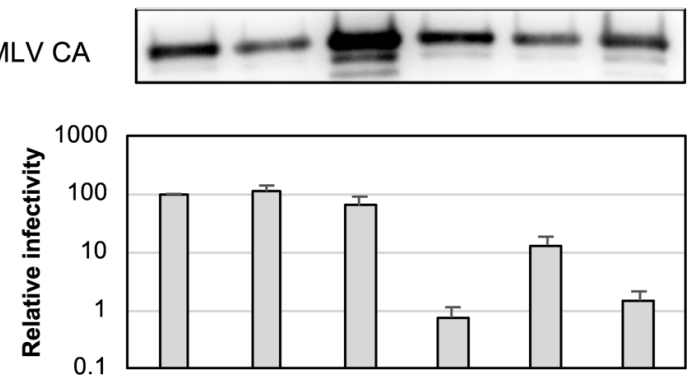

D

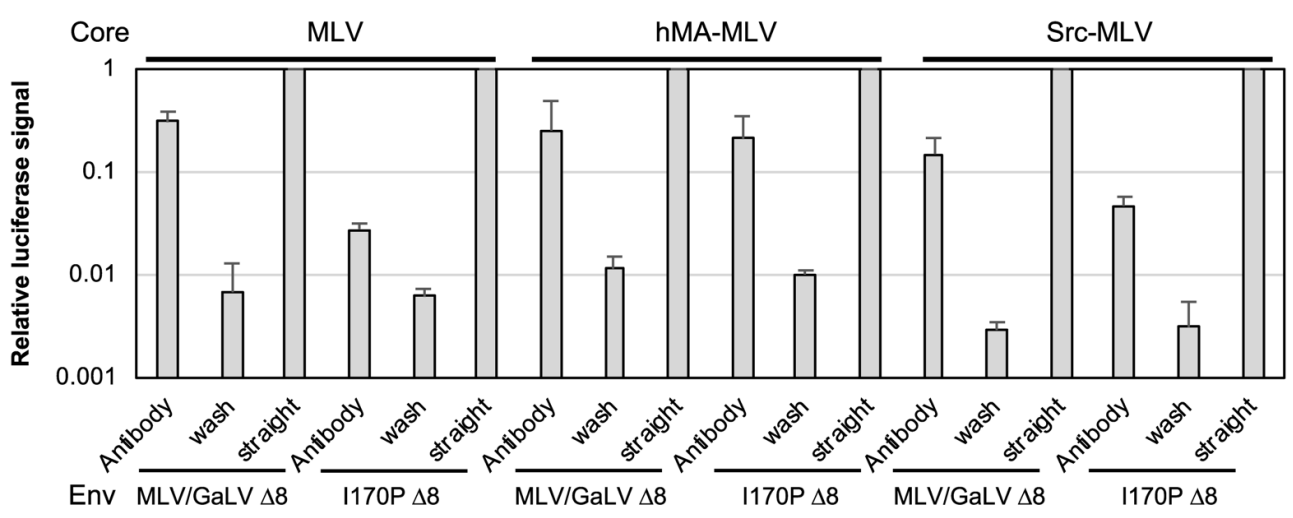


Figure 3.7: I170P Env is fusogenic out of virions but not within MLV particles.

(A) Cell-to-cell fusogenic activity of indicated Env proteins relative to MLV $\Delta 26$ Env. (B) Virus-to-cell fusogenic activity of the indicated Env proteins relative to wildtype MLV Env. Env incorporation in MLV GagCre virus by western blot. A representative example of two western blots is shown. Fusion assays represent the average and standard deviation from at least three independent experiments. $* *=\mathrm{p}<0.01 ; * * *=\mathrm{p}<0.001$; ns $=$ not significant by unpaired and two-tailed Student's $t$ test. 
Figure 3.7: I170P Env is fusogenic out of virions but not within MLV particles.
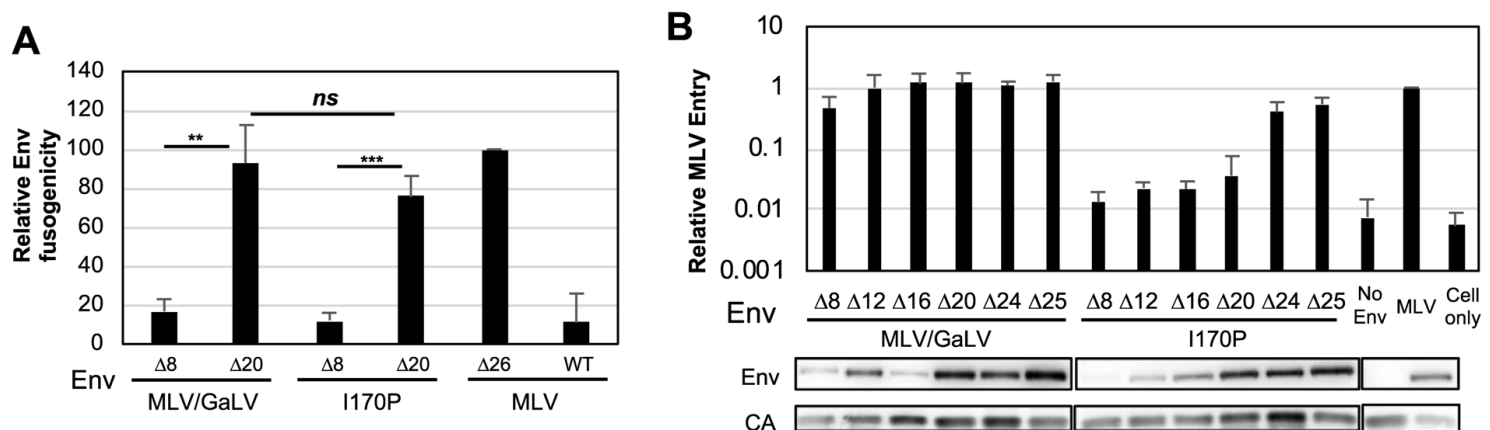
Figure 3.8: Hydrophobicity is important at the $170^{\text {th }}$ residue of $T M$.

(A) Schematic diagram of Env CT with mutations. Below is the comparison of CT from different gammaretroviruses. (B) Relative percent infectivity with mutant Env normalized to MLV/GaLV $\Delta 8$. (C) Relative percent infectivity of HIV-1, MLV, and RSV normalized to MLV/GaLV $\Delta 20$ with HIV-1. Average and standard deviation from three independent experiments for each is shown. 
Figure 3.8: Hydrophobicity is important at the $170^{\text {th }}$ residue of TM.

A

$\mathrm{MLV} / \mathrm{GaLV} \Delta 8$

I170P $\Delta 8$

MLV/GaLV $\triangle 20$

I170P $\Delta 20$

I170L

I170S

I170E

I170K

I170G

MLV

GaLV

FeLV

RD114

XMRV

Koala Retrovirus

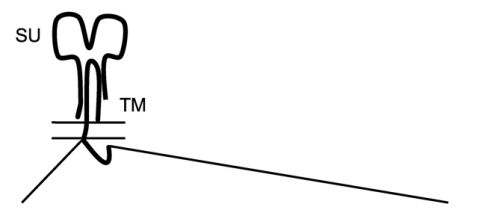

GPCIINKLVOFINDRISAVKILVLROKYQ * GPCIINKLVQFPNDRISAVKILVLRQKYQ* GPCIINKLVQFINDRIS * GPCIINKLVQFPNDRIS * GPCIINKLVQFLNDRIS* GPCIINKLVQFSNDRIS*

GPCIINKLVQFENDRIS*

GPCIINKLVQFKNDRIS*

GPCIINKLVQFGNDRIS*

GPCILNRLVQFVKDRISVVQALVLTQQYHQLKPIEYEP GPCI INKLVQF INDRISAVKILVLRQKYQALENEGNL GPCILNRLVQFVKDRISVVQALILTQQYQQIKQYDPDRP GPCVFSRLMAF INDRLNVVHAMVLAQQYQALKAEEEAQD GPCILNRLVQFVKDRISVVQALVLTQQYHQLKSIEPEEVESRE GPCVINKLVQF INDRVSAVRILVLRHKYQTLDNEDNL

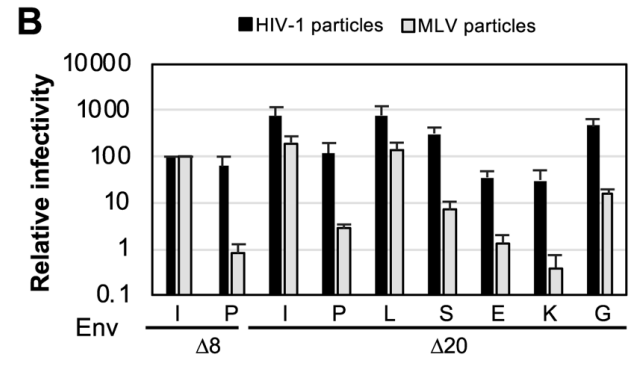

C

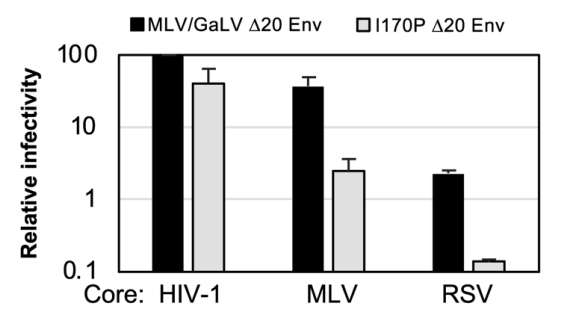


Figure 3.9: $170^{\text {th }}$ residue in MLV/GaLV Env CT is critical for MLV infectivity.

Representative Env incorporation with indicated Env from three independent experiments is shown. Average relative infectivity normalized to MLV/GaLV $\Delta 26$ (170I*) and standard deviation from three independent experiments is shown. $* * * *=p<0.0001$; ns $=$ not significant by unpaired and two-tailed Student's $t$ test. 
Figure 3.9: $170^{\text {th }}$ residue in MLV/GaLV Env CT is critical for MLV infectivity.

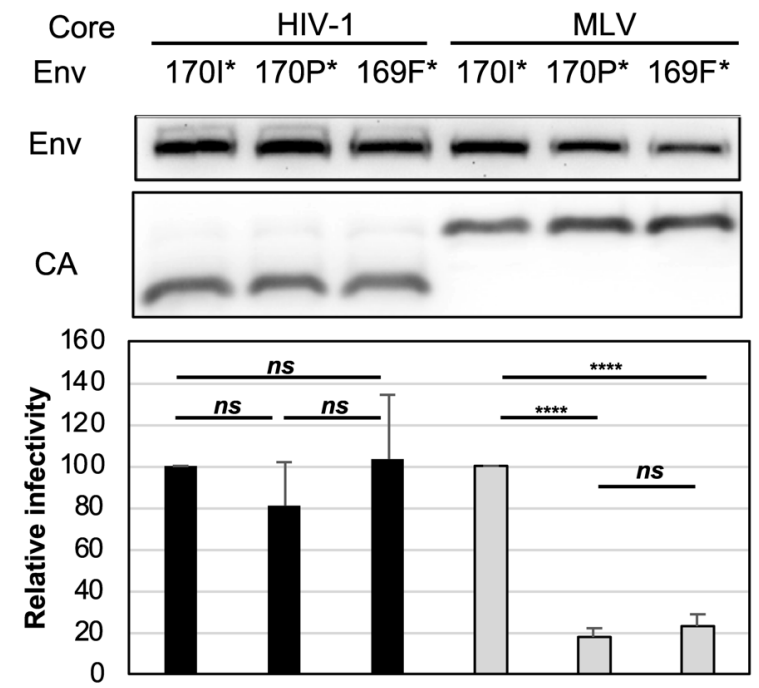




\title{
IV. Cell type specific factors dramatically affect functionality of retroviral pseudotyped particles
}

\author{
Yul Eum Song, Marc C. Johnson
}

\subsection{Abstract}

Viruses can acquire foreign envelope glycoproteins to form infectious particles through a process called pseudotyping. However, not all viruses are compatible with all glycoproteins and the factors that dictate compatibility are poorly understood. Here we analyzed the pseudotyping compatibility of ten viral glycoproteins from a diverse collection of RNA and DNA viruses with two different retroviruses, Human Immunodeficiency Virus-1 (HIV-1) and Murine Leukemia Virus (MLV), from two different cell lines, 293FT and HeLa. Interestingly, particular glycoproteins displayed up to 1,000-fold differences in compatibility depending on the virus and the cell type. These differences were found to be dictated by three main factors. The first was variability in glycoprotein expression level. Some glycoproteins required vastly more expression plasmid to reach peak infectious particle production, while others (such as HIV-1 Env) potently reduced infectious particle production when higher concentrations of expression plasmid were used. Second, some glycoproteins displayed cell-type dependence under all conditions. For instance, Rous sarcoma virus (RSV) Env was 1,000-fold less functional in HeLa cells regardless of the virus or expression level. Finally, and most surprisingly, MLV particles displayed an assembly defect in HeLa cells. This defect was independent of protein expression level, and persisted even after knocking out the restriction factor BST- 
2/tetherin alone or in combination with restriction factors ZAP, TRIM15, or TRIM25. Fusion of HeLa cells with 293FT cells alleviated the restriction, suggesting that the block was due to a cell line deficiency rather than a restriction factor. The identity of this missing factor is currently not known.

\subsection{Introduction}

Enveloped viruses can uptake foreign glycoproteins during the assembly process through a process called pseudotyping $(31,32)$. Pseudotyping has been described and studied for decades to better understand the mechanisms of viral glycoprotein acquisition and to improve vector development for gene therapy. Gammaretroviral and lentiviral vectors have been studied as desirable candidates for gene therapy due to their efficient gene transduction ability $(155,156)$.

Several factors have been reported to affect the viral glycoprotein acquisition process in retroviruses $(25,26)$. Direct interaction between retroviral structural protein (Gag) and envelope glycoprotein (Env) as well as indirect interactions through host factors or lipid membrane compartments have been proposed. For the acquisition of the native HIV-1 Env by HIV-1 particles, a direct interaction between HIV-1 Gag and the cytoplasmic tail of HIV-1 Env occurs. A trimeric structure formed by the matrix domain of HIV-1 Gag plays an important role in HIV-1 Env incorporation and functionality (143146, 148). In vitro binding of MA to the Env cytoplasmic tail from SIV and HIV-1 have also been reported $(157,158)$. Although direct interactions between viral particles and glycoproteins can be critical in some cases of Env incorporation, particularly between viruses and their native glycoproteins, several studies support that there is a more general 
envelope glycoprotein acquisition mechanism shared among diverse virus types. Retroviral Gag proteins have been proposed to interact with lipid microdomains, such as lipid raft or tetraspanin-enriched microdomains, during the glycoprotein incorporation, viral assembly, or budding process (159-162). These interactions could contribute to pseudotyping of foreign viral glycoproteins.

Several host proteins have been found to act as viral co-factors or restriction factors that affect retrovirus production in a cell type-dependent manner. For instance, the Rab coupling protein FIP1C has been reported to interact with the HIV-1 Env cytoplasmic tail and is required for Env incorporation into particles (44). Several retroviral restriction factors are also cell line specific. For instance, expression of the restriction factor APOBEC3G, a cytidine deaminase, dictates viral dependence on the HIV-1 accessory protein Vif for viral replication in a cell type-dependent manner $(19,163)$. Likewise, expression of the interferon-inducible restriction factor BST-2/tetherin dictates dependence on the HIV-1 accessory protein Vpu in a cell type-dependent manner (16). More recently, the host factors SERINC3 and SERINC5 have been shown to restrict infectivity of HIV-1 or MLV particles, making virus dependent on HIV-1 Nef and MLV glycoGag, respectively $(17,18,20)$. Different RNA nuclear export components have also been shown to affect viral assembly in a cell type-dependent manner. For instance, HIV-1 Gag assembly and budding is restricted in murine cells, but the defect can be overcome by adding a cis-acting RNA export element to the virus (164).

In this study, we examined envelope glycoprotein pseudotyping compatibility of two different retroviruses, HIV-1 and MLV, with ten diverse envelope glycoproteins, and two different producer cells, 293FT and HeLa. We found that all ten glycoproteins were generally compatible with HIV-1 and MLV particles, but some glycoproteins behaved 
differently in the two cell lines. Surprisingly, MLV particles were found to be severely restricted in HeLa cells. Further study suggests that additional unknown host factor(s) present in 293FT but not in HeLa cells is required for efficient MLV assembly.

\subsection{Materials and Methods}

Cell culture. The 293FT cell line was obtained from Invitrogen. The 293T mCAT-1 cell line expressing the ecotropic F-MLV Env receptor, mCAT-1, was kindly provided by Walter Mothes (Yale University). 293TVA cells (95) were kindly provided by John Young. 293FT cells were maintained in DMEM supplemented with $10 \%$ or $7.5 \%$ fetal bovine serum, 2mM L-glutamine, 1mM sodium pyruvate, $10 \mathrm{mM}$ nonessential amino acids, $1 \%$ MEM vitamins. For HeLa cells and its knockout derivatives, $10 \%$ FBS or $5 \%$ serum plus II(Sigma) was used for serum. The TZM-bl reporter cell line that expresses nls-GFP under the transcription control of HIV-1 LTR (TZM-GFP) is kindly provided by Massimo Pizzato (17).

Plasmids. NL4-3 derived HIV-CMV-GFP containing CMV-GFP in the place of Nef, lacking Vif, Vpr, Vpu, Nef, and Env, was kindly provided by Vineet Kewal-Ramani (National Cancer Institute [NCI]-Frederick). HIV-CMV-GFP with the Vpu gene added (HIV-CMV-GFP+Vpu) was previously described (51). CMV-MLV-GagPol and the ecotropic Friend MLV Env expression vector were kindly provided by Walther Mothes (Yale University). The MLV genome reporter constructs pQCXIP-GFP were previously described (130). The MLV GagPol with HIV-1 MA domain (hMA-MLV) was previously described (131). pHCMV-LCMV-Arm53b and pHCMV-RabiesG were gifts from Miguel 
Sena-Esteves (Addgene plasmid \#15796 and \#15785, respectively) (35). The RSV Env expression construct with the last 29 amino acids deleted ( $\triangle \mathrm{CTD}$ ) was previously described (136). The VSV-G expression construct was obtained from NIH AIDS Reagent Program (135). The B-clade consensus, codon-optimized pcDNA 3.1 HIV-1 gp160 expression construct was kindly provided by Beatrice Hahn (University of Pennsylvania) (165). Viral glycoprotein constructs expressing LASV GP, GP64, or CHIKV E glycoproteins are kindly provided by Wendy Maury. The Ebola GP construct was kindly provided by David Sanders. LentiCRISPR v2 and lentiCas9-Blast were gifts from Feng Zhang (Addgene plasmid \# 52961 and 52962, respectively) (166).

Generation of CRISPR-Cas9 induced knockout cells. The tetherin knockout HeLa cell line (HeLa $\Delta$ Tetherin) was generated by transduction with LentiCRISPR v2 containing the gRNA sequence: 5'-GCGCTTATCCCCGTCTTCCA -3'. After selection with puromycin, single cells were isolated by limiting dilution and expanded. For the additional knock out, HeLa $\Delta$ Tetherin cells were transfected with LentiCRISPR v2 with selected guide RNA along with lentiCas9-Blast for selection. After 24 hours, transfected cells were selected with blasticidin for 2 days, and single cells were isolated and expanded. The gRNA sequences used were: 5'-GCCCGCAACCCCGTCCCTGA-3' for TRIM15, 5'GAGCCGGTCACCACTCCGTG-3' for TRIM25, and 5'GGCCGGGATCACCCGATCGG -3' for ZAP. Gene disruption in each case was verified by PCR amplification of gRNA target site in the gDNA with flanking primers and directly sequencing the PCR product. 
Infectivity assay. Transfections for infectivity and western blotting were performed using polyethylenimine (PEI) (137) in 293FT cells and jetPRIME (Polyplus transfection) in HeLa cells. Except where noted, transfections were performed in 6-well plates with 900ng of HIV-CMV-GFP, HIV-CMV-GFP+Vpu, or 450ng of CMV-MLV-GagPol plus 450ng of pQCXIP-GFP and 100ng of viral envelope glycoprotein. In some cases the transfections were performed in 12 well plates and scaled as appropriate. For the titration assay, 250ng of HIV-CMV-GFP or 125ng of CMV-MLV-GagPol and 125ng of pQCXIP-GFP were transfected with 4ng, 16ng, 64ng, or 256ng of viral envelope glycoproteins and pUC19 plasmid DNA was used to normalize total DNA quantity. Medium was changed about 24 hours post-transfection and collected after another 24 hours. Supernatant containing the virus was frozen at $-80 \mathrm{C}^{\circ}$, thawed, spun at $3,200 \mathrm{xg}$ for 5 minutes, and transferred onto target cells with 20ug of hexadimethrine bromide per ml (Sigma H9268). For infectivity with HIV-1 Env, TZM-GFP cells were used as target cells. For RSV Env, 293 TVA cells were used as target cells. For the rest of viral glycoproteins, 293T mCAT-1 cells were used as target cells. Infected cells were collected about 48 hours later, fixed with $4 \%$ paraformaldehyde, washed with PBS, and analyzed on an Accuri C6 flow cytometer. Infectious units per $\mathrm{ml}$ produced was calculated from MOI.

Western blotting. Transfected cells were lysed in RIPA buffer (NaCl 150mM, NP-40 1\%, Sodium deoxycholate $0.5 \%$, SDS $0.1 \%$, Tris $25 \mathrm{mM}$ ) with Protease inhibitor (A32955, ThermoScientific), clarified by brief centrifugation, and mixed with sample buffer (final concentration: $50 \mathrm{mM}$ Tris, $2 \%$ sodium dodecyl sulfate [SDS], 20\% glycerol, $5 \%$ ßmercaptoethanol). For probing tetherin/BST-2 expression in HeLa cells and the HeLa $\Delta$ Tetherin cells, cell lysates were additionally treated with PNGaseF (NEB) to remove N- 
linked oligosaccharides from tetherin. 5\% of whole cell lysate was heated for 5 minutes in $95 \mathrm{C}^{\circ}$ and loaded on a $10 \%$ SDS-PAGE gel, and transferred to a $0.45 \mathrm{um}$ PVDF. For viral samples, $1 \mathrm{ml}$ of viral media were concentrated by centrifugation at $14,000 \mathrm{rpm}$ for 3 hours on $20 \%$ sucrose cushion. Viral pellets were resuspended in sample buffer and denatured by heating at $95 \mathrm{C}^{\circ}$. For probing capsid, viral samples were loaded on a $10 \%$ SDS-PAGE gel and transferred to $0.45 \mathrm{um}$ PVDF. For probing HIV-1 MA, viral samples were loaded on a $15 \%$ SDS-PAGE gel and transferred to 0.2um PVDF. For detecting viral proteins, membranes were blocked with $2 \%$ nonfat dry milk and incubated with primary or secondary antibodies in $1 \%$ nonfat dry milk. For detecting cell lysates, blocking and antibody incubation were all done in 5\% nonfat dry milk. Anti-HIV p24 hybridoma medium [183-H12-5C] from Bruce Chesbro (138) was used at 1:500 dilution. MLV capsid was detected by using anti-MLV capsid medium from hybridoma (R187, ATCC) (139) at 1:250 dilution. HIV-1 p17 antiserum was obtained from NIH AIDS reagent program, Division of AIDS, NIAID, NIH: Anti-HIV-1 p17 polyclonal from Dr. Paul Spearman and Dr. Lingmei Ding (167). GAPDH was detected for loading control in cell lysates (Santa Cruz Biotechnology SC-47724). Tetherin/BST-2 antibody was obtained from NIH AIDS Reagent program, Division of AIDS, NIAID, NIH: Anti-BST-2 Polyclonal (\#11721) from Drs. Klaus Strebel and Amy Andrew (55). HRP-conjugated secondary antibodies were used in 1:10,000 dilution in all blots. Horseradish peroxidase-linked anti-mouse (A5378), anti-rat (A5795), anti-rabbit (A0545) were obtained from Sigma. Luminata Classico Western HRP substrate (Millipore) was used for visualization of the membranes with a chemiluminescence image analyzer UVP BioSpectrum 815 Imaging System. 
Immunofluorescence microscopy. 293FT cells or HeLa $\Delta$ Tetherin cells were plated on a glass bottom dish (MatTek) and transfected with 900ng of HIV-CMV-GFP or 450ng of hMA-MLV and 450ng of pQCXIP-GFP with 100ng of pHCMV-LCMV-Arm53b. After 48 hours, transfected cells were fixed with $4 \%$ paraformaldehyde, washed, and permeabilized with $0.1 \%$ Triton X-100 and blocked with $10 \%$ goat serum for 1 hour. After washing, cells were incubated with Anti-HIV-1 p17 polyclonal (167) for 1 hour, washed, and incubated with alexa 555 conjugated a-Rabbit secondary antibody (Life technology, A21428) for 1 hour. After washing, nuclei were stained with Hoechst 33342 (Thermo Scientific), washed, and immuno-stained cells were observed on confocal microscope (Leica TCS SP8, Molecular Cytology Core, University of Missouri).

Syncytium induction assay. 293FT cells or HeLa $\Delta$ Tetherin cells were transfected with lug of virus (HIV-CMV-GFP or CMV-MLV-GagPol plus pQCXIP-GFP) or 1ug of VSVG construct. Twelve hours later, cells transfected with one of viruses were co-cultured with cells transfected with VSV-G. Twelve hours later, co-cultures were incubated with pH 6 DMEM for 10 minutes, the medium was exchanged, and the virus-containing supernatant was collected after 24 hours for infection.

\subsection{Results}

Viral glycoproteins exhibit cell type-dependent compatibility. In our studies of factors that affect compatibility between particular viruses and glycoproteins, we noted several cases where the functionality of particular glycoproteins appeared to be virus or cell-type dependent. For instance, when we produced HIV-1 particles pseudotyped with 
glycoproteins from a rhabdovirus (vesicular stomatitis virus, VSV-G), a lentivirus (HIV-1 Env), a baculovirus (GP64), or an arenavirus (Lassa virus, LASV GP, or lymphocytic choriomeningitis, LCMV GP), we observed distinct cell type-dependent differences in infectious particle production (Figure 4.1A). While the HIV-1 particles pseudotyped with VSV-G were much more efficiently produced from 293FT cells, the particles pseudotyped with HIV-1 Env, LASV GP, and LCMV GP were all more efficiently produced from HeLa cells. However, when the same five glycoproteins were tested with the gammaretrovirus MLV, all five glycoproteins were more efficiently produced from 293FT cells (Figure 4.1B). This was initially believed to be due to the restriction factor BST-2/tetherin, which is constitutively expressed from HeLa cells $(16,62)$. While the HIV-1 construct used in these experiments contained Vpu, which can counteract BST-2/tetherin, the MLV particles did not have a mechanism to counteract the restriction factor. It should be noted, the MLV GagPol construct used in all experiments lacked the 5' leader sequence required for expression of the glycoGag accessory gene (7), thus only the Gag and GagPol proteins were produced. Not surprisingly, the lowest titer came from MLV particles pseudotyped with HIV-1 Env as the long cytoplasmic tail of HIV-1 Env has been shown to preclude incorporation into MLV particles (168).

To further investigate this cell-type dependence of MLV particles with various viral glycoproteins independent of BST-2/tetherin, we chose to generate a clonal HeLa BST2/tetherin knockout cell line (Figure 4.2A). CRISPR/Cas9 with a gRNA targeting exon 1 of BST-2/tetherin gene was used to generate knockout cells. After 2 rounds of isolation, we selected a knockout clone with a mixture of $+1,+2$, and -4 indels, suggesting that all open reading frames were disrupted and that the cell line has at least three copies of chromosome 19, where the BST-2/tetherin gene is located. This cell line was also verified 
for knockout of the protein expression by western (Figure 4.2A). Next, the knockout cell line (HeLa $\Delta$ Tetherin) was functionally tested to determine if the HIV-1 accessory protein Vpu was no longer required for infectious particle production (Figure 4.2B). In HeLa cells, HIV-1 infectious particle production was approximately 10-fold higher in the presence of Vpu, while Vpu had little effect on infectious particle production in 293FT cells, which do not constitutively express BST-2/tetherin. In contrast, infectious HIV-1 particle production from HeLa $\Delta$ Tetherin cells was generally not affected by Vpu. For particles produced with VSV-G and LASV GP, Vpu did not significantly enhance infectious particle production. However, the infectivity with some viral glycoproteins was partially enhanced by Vpu, suggesting that Vpu may have other targets that can affect infectious particle production in HeLa cells, but the enhancement was similar to that seen in 293FT cells and generally less than the enhancement observed from wildtype HeLa cells. Notably, the infectious MLV particle production from HeLa $\Delta$ Tetherin cells remained quite low, and little difference between HeLa $\Delta$ Tetherin cells and parent HeLa cells was observed, suggesting that factors other than BST-2/tetherin restrict MLV infectious particle production.

\section{Cell type-dependent compatibility is partially dependent on viral glycoprotein}

expression level. Next, we sought to investigate the pseudotyping compatibility of different envelope glycoproteins in 293FT and HeLa $\Delta$ Tetherin cells. Several studies reported envelope glycoproteins from various viral families efficiently produced pseudotyped retroviruses in HEK 293 cells $(33-37,136,169)$. We chose 10 different viral envelope glycoproteins from different families known to be compatible with retroviruses to test cell-line and virus-type dependent compatibility (Figure 4.3). Additional envelope glycoproteins chosen were from retroviruses (MLV Env and RSV Env), rabdoviruses 
(Rabies virus, RVG), alphavirus (chikungunya virus, CHIKV glycoprotein), and filovirus (Ebola GP). For more efficient viral particle production, Ebola GP without the mucin-like domain and RSV Env without cytoplasmic tail were used $(95,170)$. In each case, we used a constant amount of plasmid to produce the viral particles but varied the amount of viral glycoprotein (Figure 4.3). Among the 10 viral envelope glycoproteins tested, most glycoproteins efficiently produced infectious HIV-1 particles ( $>10^{5}$ infectious particles $\left./ \mathrm{ml}\right)$ from both cell types, though titers varied considerably depending on the cell line and the transfection amounts (Figure 4.3A). The highest level of infectious HIV-1 particle production from both cell lines was obtained with VSV-G $\left(>10^{7}\right.$ infectious particles $\left./ \mathrm{mL}\right)$, although it took considerably more VSV-G plasmid to reach this titer from HeLa $\Delta$ Tetherin cells than from 293FT cells. With most of the glycoproteins in 293FT cells, the HIV-1 infectious particle production was reduced up to 100 -fold when larger amounts of the glycoprotein expression vector were used. This observation helps explain why some glycoproteins initially appeared to be less functional in 293FT cells. The glycoprotein that showed the clearest cell-type specificity was RSV Env, which could produce $>10^{6}$ infectious HIV-1 particles/mL from 293FT cells, but only about $10^{3}$ infectious HIV-1 particles $/ \mathrm{mL}$ from HeLa $\Delta$ Tetherin cells.

For infectious MLV particle production, cell type dependent viral envelope glycoprotein compatibility generally follows as HIV-1 (Figure 4.3B). MLV particles pseudotyped with VSV-G produced the highest titer of the 10 glycoproteins from both cell lines. However, unlike HIV-1/VSV-G particles which reach similar titers from 293FT and HeLa $\Delta$ Tetherin cells, the maximum MLV/VSV-G titer was 40-times lower from HeLa $\Delta$ Tetherin cells than from 293FT cells. This phenotype is most clearly seen when the maximum infectivity of each viral glycoprotein from each condition tested is compared 
between HIV-1 and MLV in each cell line (Figure 4.4). Like with HIV-1, MLV particles pseudotyped with RSV Env failed to produce detectable infectious particles from $\mathrm{HeLa}$ $\Delta$ Tetherin cells. In 293FT cells, some glycoproteins (MLV Env and LCMV GP) produced similar maximum titers with HIV-1 and MLV particles. However, in HeLa $\Delta$ Tetherin cells the HIV-1 titer was higher than the MLV titer (as much as 400-fold higher) with all 10 glycoproteins.

MLV particle release is restricted in HeLa cells. To investigate whether the lower MLV titers from HeLa $\Delta$ Tetherin cells was due to restricted viral release, we sought to compare the viral release of HIV-1 and MLV particles from 293FT cells and HeLa $\Delta$ Tetherin cells. An MLV Gag chimera containing HIV-1 MA in the place of MLV MA (hMA-MLV) was used for comparison in order to directly compare the protein expression level by probing HIV-1 MA (Figure 4.5A). The LCMV GP was chosen in these experiments because it produced relatively similar titers with both viruses (Figure 4.4). HIV-1, MLV, and hMAMLV were all found to bud efficiently from 293FT cells, and the infectivity of hMA-MLV was a few-fold lower than wildtype MLV. However, MLV particles were found to bud less efficiently from HeLa $\Delta$ Tetherin cells than HIV-1 particles, and the hMA-MLV particles were found to bud almost not at all, despite displaying higher protein expression levels in the cells. While the infectious HIV-1 particle production from HeLa $\Delta$ Tetherin cells was similar to that from 293FT cells, the infectious particle production for MLV and hMAMLV particles was reduced 10 -fold and 100-fold from HeLa $\Delta$ Tetherin cells, respectively. Notably, MLV infectious particle production from 293FT cells was about 10-times higher than HIV-1 infectious particle production under this condition, which uses more structural protein expression vectors. 
ZAP, TRIM25, and TRIM15 do not contribute MLV restriction in HeLa cells. Several host factors have been reported that could restrict MLV production in HeLa cells. The tripartite motif (TRIM) family of proteins have been reported to have antiviral activity against multiple viruses (171). The most studied TRIM protein is TRIM5 $\alpha$ from Old World monkey, which is a potent HIV-1 restriction factor (172). Human TRIM15 was also shown to have antiviral activity against $\operatorname{HIV}-1$ and $\operatorname{MLV}(173,174)$. Overexpression of human TRIM15 restricted MLV infectivity and release in HEK293 cells, and HeLa cells treated with siRNA targeting TRIM15 increased MLV infectivity. To test the hypothesis that TRIM15 is responsible for the MLV restriction in HeLa cells, we proceeded to generate an additional knockout in HeLa $\Delta$ Tetherin cells (Figure 4.6A). However, the double knockout cells did not display enhanced hMA-MLV infectivity or release (Figure 4.6B, 6C).

In a recent study, a zinc-finger antiviral protein (ZAP) was shown to deplete unspliced RNAs with multiple CG-dinucleotides (175). In this study, the reporter gene expression was affected by CG dinucleotide in HeLa cells, and ZAP knockout overcame the restriction. Because the HIV-1 genome is mostly deficient in CG dinucleotides but MLV is not, we hypothesized that antiviral activity of ZAP restricts MLV in HeLa cells. In addition to this, TRIM25 was also hypothesized to affect MLV infectivity since it was reported to be essential for antiviral activity of ZAP $(176,177)$. TRIM25 was also found in a previous study to affect MLV LTR expression and infectivity (173). We generated additional knockouts of ZAP or TRIM25 in the HeLa $\Delta$ Tetherin cells to test these hypotheses (Figure 4.6A). Surprisingly, neither of these knockouts restored MLV infectivity or release from HeLa cells (Figure 4.6B, 6C). 
hMA-MLV is assembly defective in HeLa cells. Next, we analyzed the cellular distribution of hMA-MLV Gag expression in cells by immunofluorescence microscopy (Figure 4.7). HIV-1 Gag proteins were shown to be enriched on the plasma membrane with discrete puncta in both 293FT cells and HeLa $\Delta$ Tetherin cells. However, hMA-MLV exhibited distinctly different phenotypes in the two cell lines. While distinct puncta appear on the plasma membrane in 293FT cells, almost no puncta or Gag enrichment on the plasma membrane were found in HeLa $\triangle$ Tetherin cells, suggesting that hMA-MLV is assembly defective in HeLa cells. We also observed released viral particles on the coverslip for all samples except the hMA-MLV in HeLa $\Delta$ Tetherin cells (data not shown). Notably, the cytosolic signal from hMA-MLV is stronger than HIV-1 in both cell lines, which is also correlated to the higher hMA-MLV Gag expression in cells (Figure 4.6).

Host factor deficiency restricts hMA-MLV production in HeLa cells. To investigate whether the hMA-MLV assembly defect in HeLa $\triangle$ Tetherin cells is due to a restriction factor present in HeLa cells, or lack of a necessary host factor present in 293FT cells, we conducted assays where the two cell types were fused together (Figure 4.8). Briefly, 293FT cells and HeLa $\Delta$ Tetherin cells were transfected individually with either virus (HIV-1 or hMA-MLV) or VSV-G. Next, each virus producing cell population was co-cultured with cells expressing VSV-G (293FT or HeLa), and pH-shocked to induce VSV-G-mediated fusion. Infectious particles could only be produced when virus expressing cells fused with VSV-G expressing cells. All eight sets of co-cultures exhibited syncytia and significant infectious particle production. Strikingly, when HeLa $\Delta$ Tetherin cells were transfected with hMA-MLV, the infectious particle production was 70-fold higher if the cells were fused with 293FT cells expressing VSV-G than if they were fused with HeLa $\Delta$ Tetherin 
cells expressing VSV-G. This observation suggests that the MLV assembly defect in HeLa $\Delta$ Tetherin cells is not due to a restriction factor, but rather the lack of a necessary assembly co-factor that is present in 293FT cells.

\subsection{Discussion}

Pseudotyping of retroviruses has been studied not only to generate safe and efficient retroviral vectors for gene therapy, but also to study the entry mechanism and for the development of vaccines against agents such as Ebola viruses in less restrictive conditions (37). Extensive research has been performed studying the mechanisms of pseudotyping, yet gaps still remain especially regarding cellular factors involved.

We present here an analysis of the pseudotyping compatibility of ten different viral glycoproteins from diverse viral families with two different retroviruses (HIV and MLV) and two different cell lines (HeLa and 293FT). Initially the different envelope glycoproteins appeared to display noticeable differences in infectious units production between the two cell lines, most of these differences could be overcome by optimizing the ratio of virus to glycoprotein in each cell line. As expected, MLV particles could not be efficiently pseudotyped with HIV-1 Env, presumably because the long cytoplasmic tail of HIV-1 Env restricted incorporation into MLV particles (168). Unexpectedly, RSV Env without its cytoplasmic tail was compatible with HIV-1 or MLV in 293FT cells, but incompatible with both viruses in HeLa cells. This finding suggests that RSV Env is either incorrectly formed in or is unable to traffic to the virus assembly sites in HeLa cells.

In general, the ten glycoproteins exhibit a similar trend in compatibility with either HIV-1 and MLV, with MLV Env and VSV-G producing the highest titers with both viruses. 
MLV particle infectivity was generally restricted when produced in HeLa cells. This was initially believed to be because MLV release in HeLa cells is highly restricted due to the constitutively expressed tetherin (178). Generation of a BST-2/tetherin HeLa knockout cell line did not restore MLV infectivity, though it did largely alleviate the Vpu-mediated enhancement of HIV-1 infectivity. This MLV defect in HeLa cells was found to be related to viral assembly and release, and not a defect in Gag expression. Replacement of the MLV MA domain with HIV MA (hMA-MLV) reinforced the defect and resulted in a near complete loss in viral release from HeLa cells, despite the same construct displaying normal levels of infectious particle release from 293FT cells. The immunostaining study shows that hMA-MLV expression is higher, but the assembly is clearly disrupted in HeLa $\Delta$ Tetherin cells.

The budding defect of hMA-MLV in HeLa cells is reminiscent of an HIV-1 Gag assembly defect in mouse cells. However, the HIV-1 defect appeared to be due to the lack of proper RNA nuclear export element and could be alleviated by incorporating an MPMV RNA export element (164). Adding HIV-1 RRE into the hMA-MLV construct and coexpressing of Rev did not restore infectivity in HeLa cells (data not shown). However, the construct still contained wildtype MLV Pol sequence which has been shown to contain cisacting RNA sequences that affect translation could potentially negatively affect proper translation if the proper co-factors are not present in HeLa cells. It has been well established that HIV-1 RRE and Rev utilizes CRM1-mediated nuclear export pathway and MPMV CTE (constitutive transport element) uses NXF1/NXT pathway $(23,179)$. RNA element in MLV is not clearly defined, but translation enhancing sequences in Pol were found to be enhanced by NXF1/NXT expression (180). However, overexpression of the 
NXF1/NXT host factors did not enhance hMA-MLV infectivity significantly in HeLa cells (data not shown).

Further studies suggested that host restriction factors do not significantly contribute to the MLV restriction in HeLa cells. In previous reports, overexpression of TRIM15, ZAP, or TRIM25 in HEK293 cells displayed antiviral effects $(173,176,177)$. In HeLa cells, siRNA targeting TRIM15 enhanced MLV infectivity, and siRNA targeting ZAP or CRIPSR-induced ZAP knockout overcame the restriction on the CG-dinucleotide enriched RNA $(173,175)$. Knockout of TRIM15, TRIM25, or ZAP in HeLa cells all failed to enhance the infectivity hMA-MLV from HeLa cells. However, fusion of MLV expressing HeLa cells with 293FT cells enhanced infectious particle production by 70 -fold, suggesting that the assembly defect is due the lack of a critical factor present in 293FT cells but absent in HeLa cells.

Overall, we studied multiple different viral envelope glycoprotein compatibility with HIV-1 and MLV in two different cell lines, 293FT and HeLa cells. Generally various envelope glycoproteins produced infectious particles of HIV-1 and MLV with a few exceptions. Unexpectedly MLV assembly and release is restricted in HeLa, suggesting the two viruses have different cellular co-factor requirements, and a critical MLV co-factor is missing from HeLa cells. Further studies are required to determine the identification of this essential co-factor. 
Figure 4.1: Viral glycoprotein compatibility is dependent on cell types.

900ng of vectors expressing virus and 100ng of vectors expressing glycoproteins were used for transfection. Average and standard deviation of infectious units produced per $\mathrm{ml}$ from 3 independent experiments is shown for HIV-1 (A) and MLV (B). 
Figure 4.1: Viral glycoprotein compatibility is dependent on cell types.

A.

HIV-1 infectivity

口293FT cells $\square$ HeLa cells

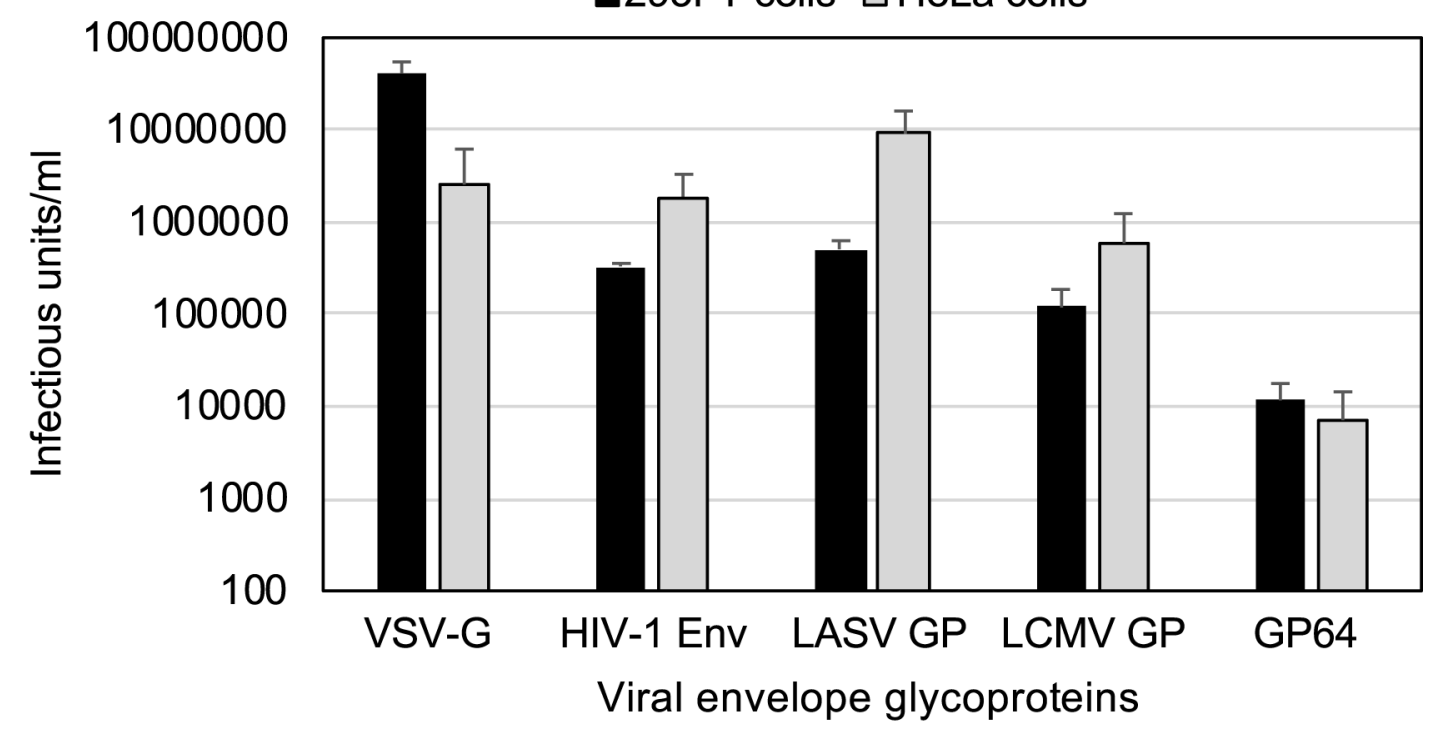

B.

MLV infectivity

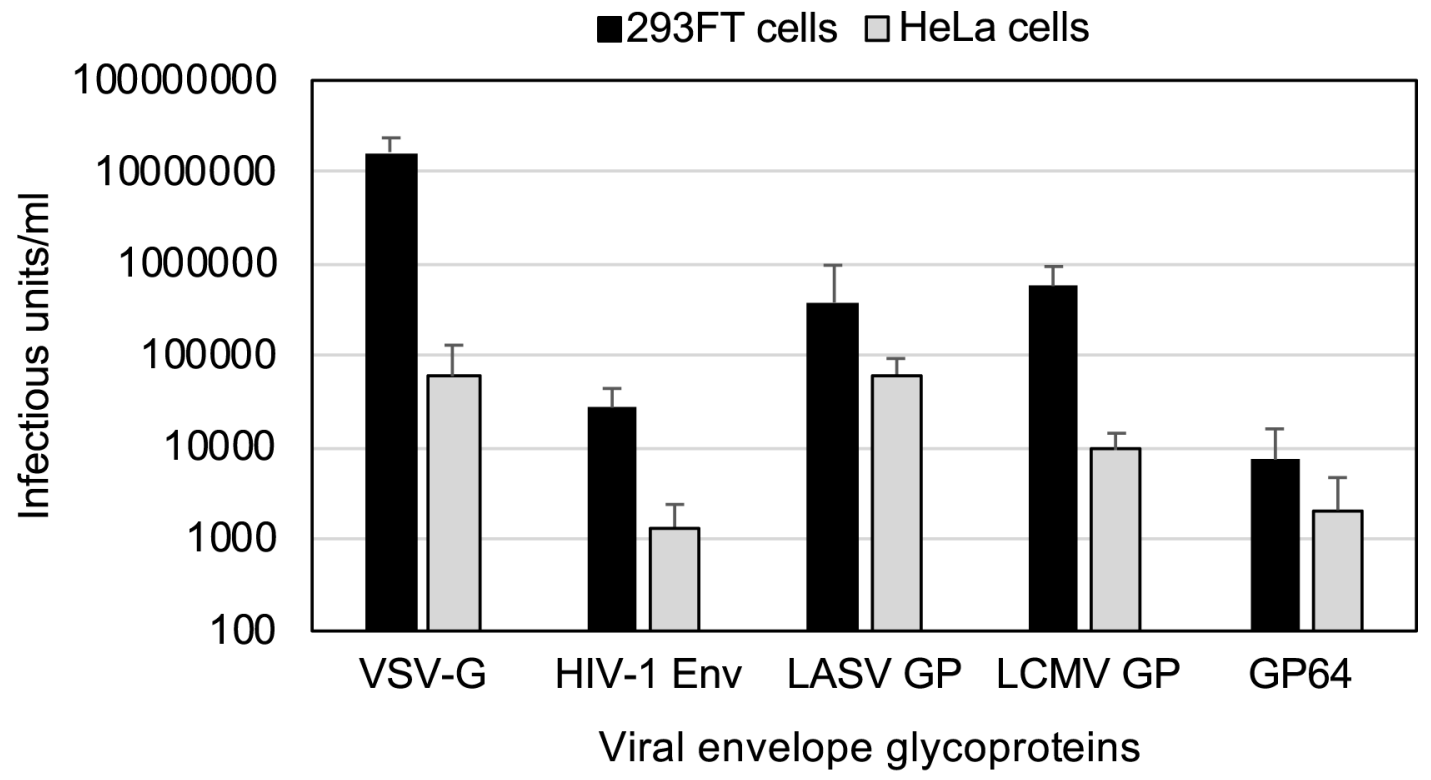




\section{Figure 4.2: Effect of tetherin knockout in HeLa cells.}

(A) Confirmation of CRISPR/Cas9-induced tetherin knockout in HeLa cells. Genomic DNA was extracted from parent HeLa cells and HeLa $\Delta$ Tetherin cells, PCR amplified with flanking primers containing the target guide RNA region, and sequenced. Sequencing result reveals three indel variations $(+1,+2,-4)$ in HeLa $\Delta$ Tetherin cells. Western blotting shows the tetherin expression is knocked out in HeLa $\Delta$ Tetherin cells. (B) Average and standard deviation of Infectious units per $\mathrm{ml}$ of three different viruses (HIV1 (Vpu-), HIV-1 (Vpu+), MLV) produced in FT, HeLa, HeLa $\Delta$ Tetherin cells are shown from 3 independent experiments. Transfection was performed as in Figure 4.1. 
Figure 4.2: Effect of tetherin knockout in HeLa cells.

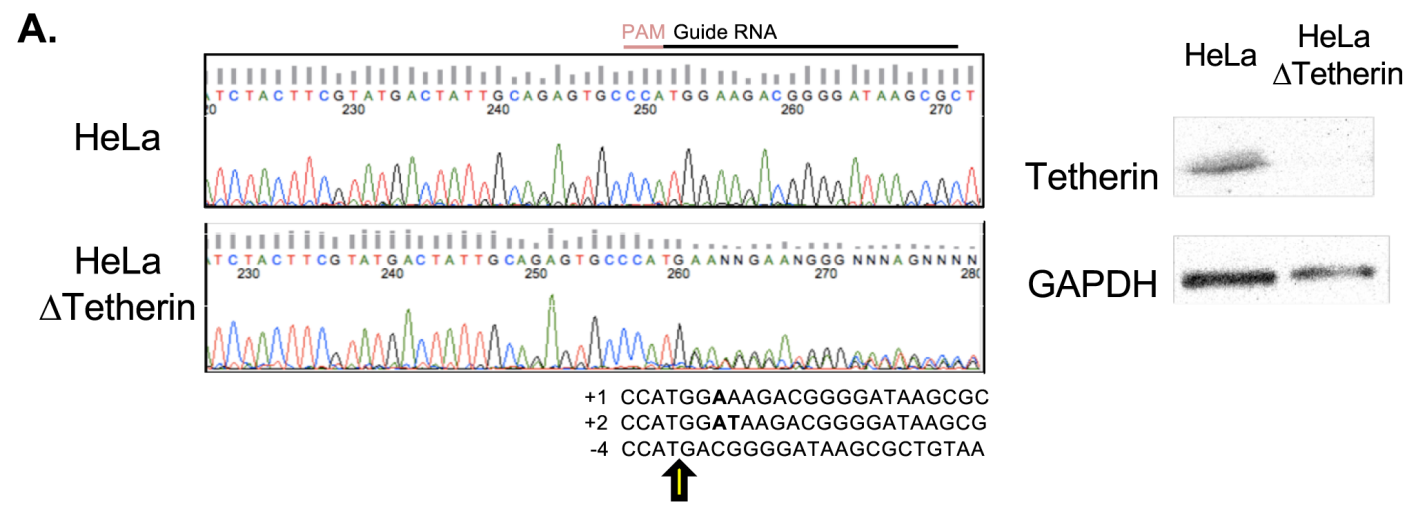

B.

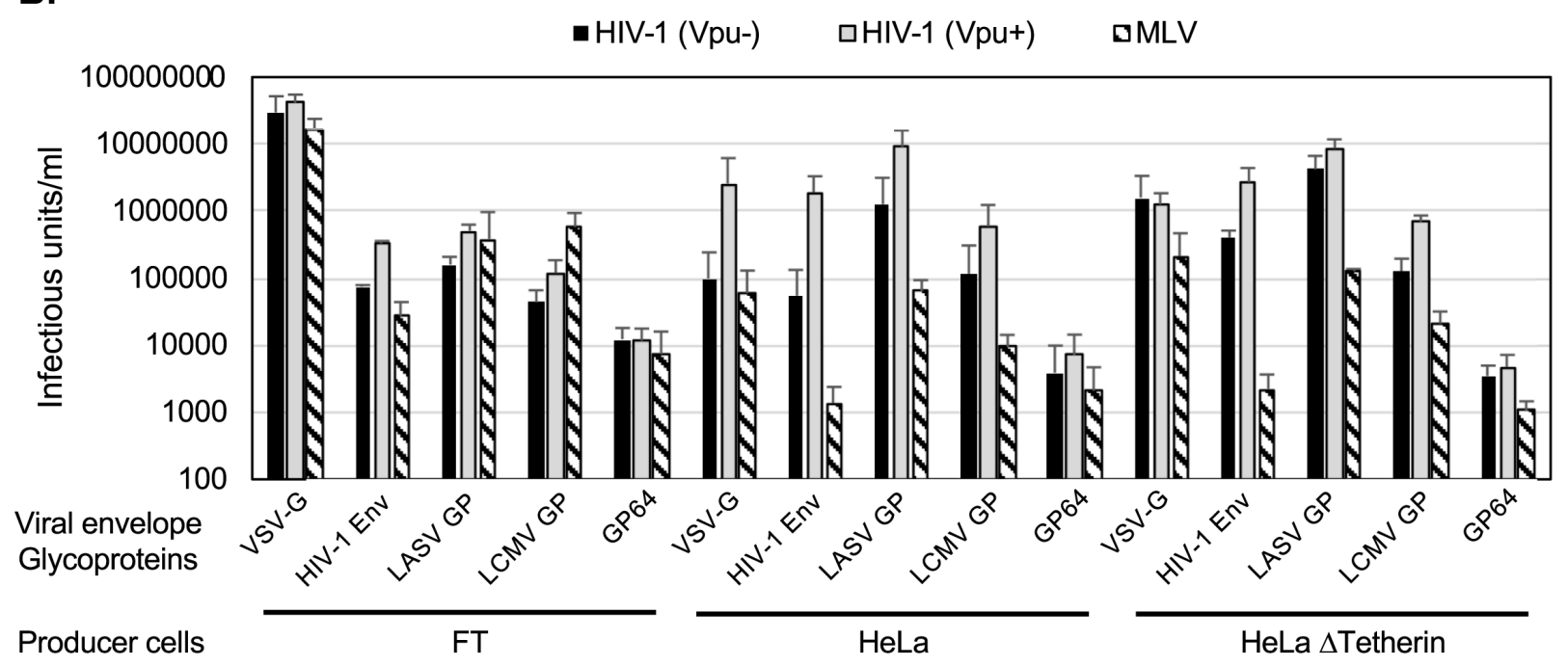


Figure 4.3: Cell type specific Env incompatibility is partially dependent on Env expression level.

Average and standard deviation of HIV-1 (Vpu-) (A) and MLV (B) infectious units per $\mathrm{ml}$ produced in $293 \mathrm{FT}$ and HeLa $\Delta$ Tetherin cells with 10 different viral glycoproteins. Increasing amounts of glycoproteins (4ng, 16ng, 64ng, 256ng) were used per each glycoprotein with a consistent amount of virus (250ng). Shown are from 3 independent experiments. 
Figure 4.3: Cell type specific Env incompatibility is partially dependent on Env expression level.

A.

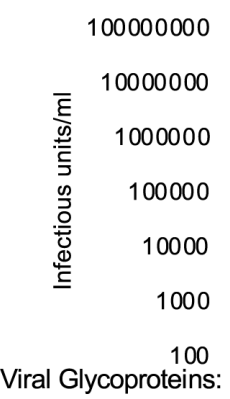

HIV-1 (IU/ml)

- 293FT cells $\square$ HeLa $\Delta$ Tetherin cells

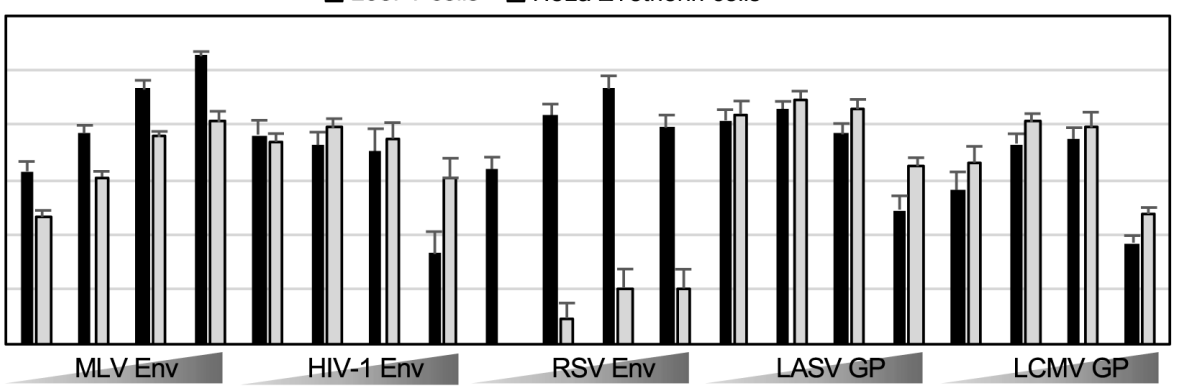

HIV-1 (IU/ml)

293FT cells $\square$ HeLa $\Delta$ Tetherin cells

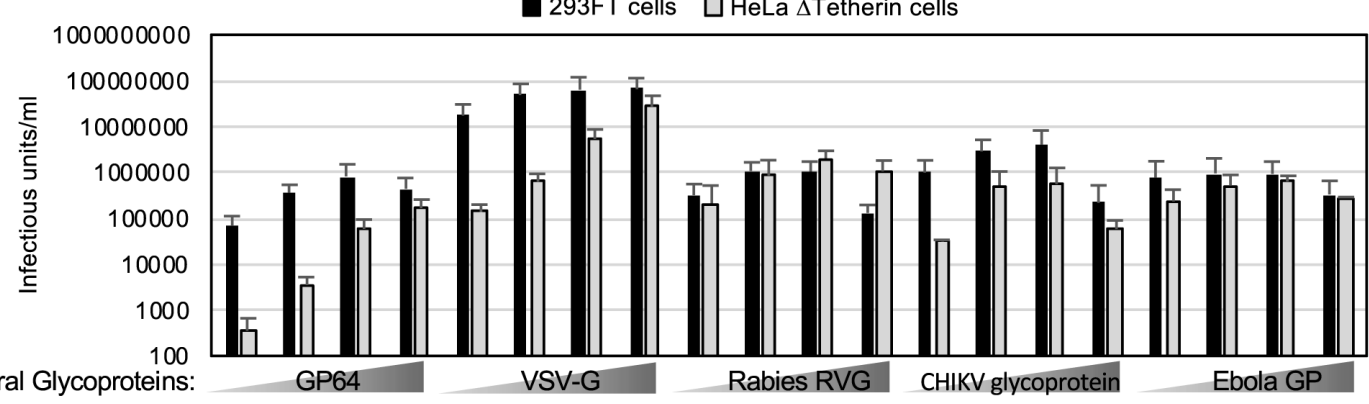

B.

MLV (IU/ml)

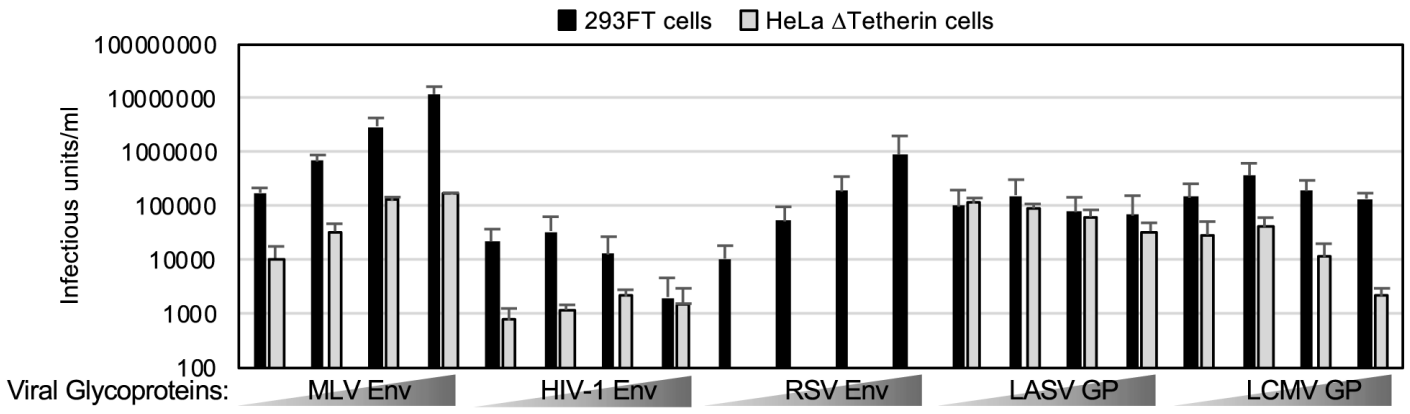

$\mathrm{MLV}(\mathrm{IU} / \mathrm{ml})$

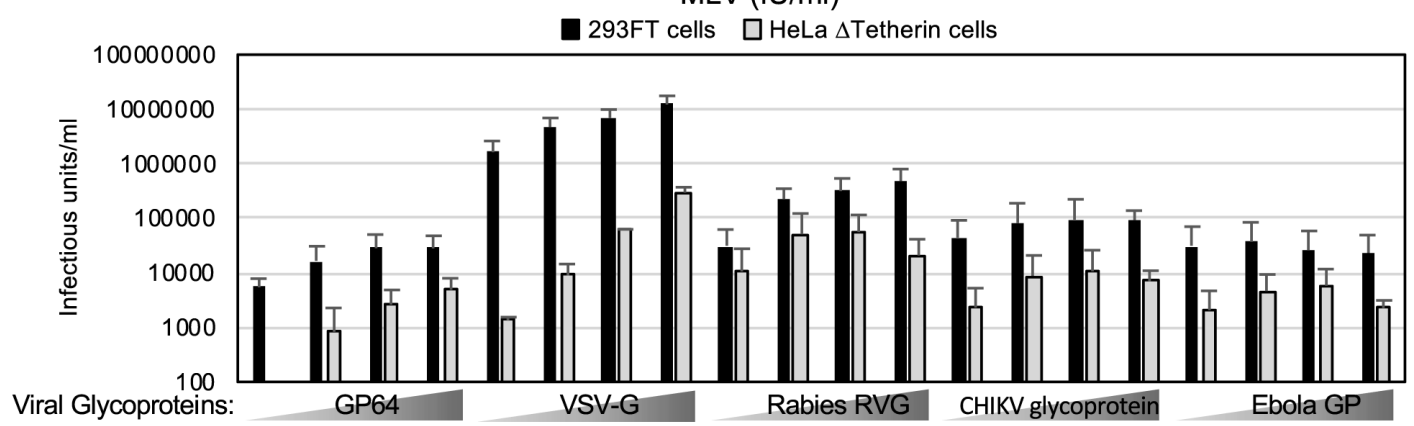


Figure 4.4: MLV infectious units production is restricted in HeLa $\triangle$ Tetherin cells.

The highest infectivity from Figure 4.3 was selected and plotted by cell types. 
Figure 4.4: MLV infectious units production is restricted in HeLa $\Delta$ Tetherin cells.

Producer cells: 293 FT

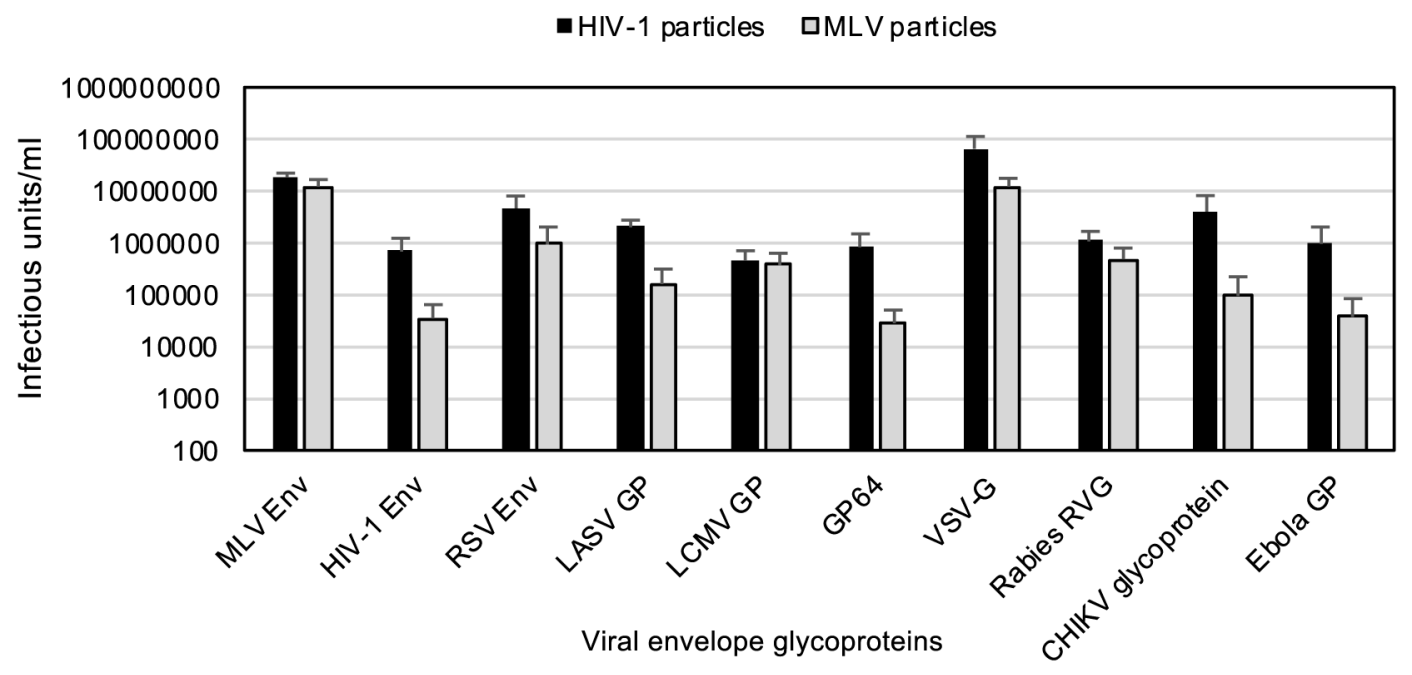

Producer cells: HeLa $\Delta$ Tetherin cells

- HIV-1 particles aMLV particles

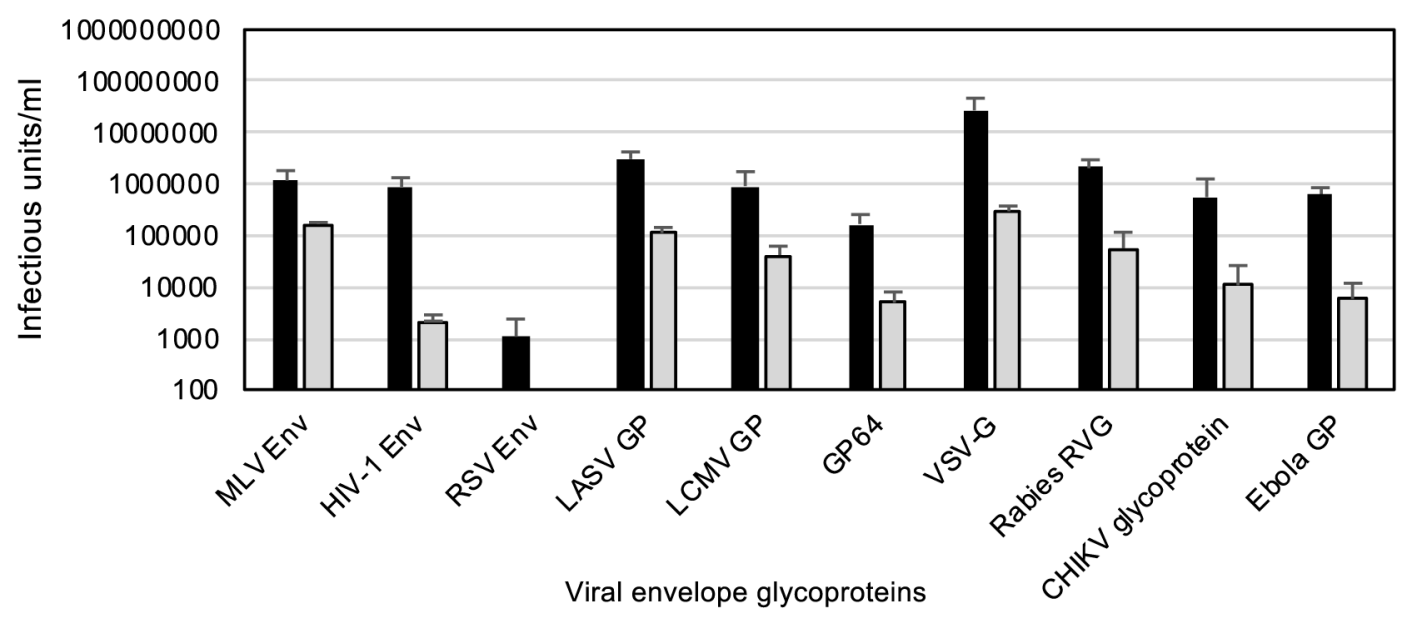


Figure 4.5: MLV release is restricted in HeLa $\triangle$ Tetherin cells.

(A) Schematic of HIV-1, MLV, and HIV-1 MA-MLV Gag. (B) 293FT cells and HeLa $\Delta$ Tetherin cells were transfected with HIV-1, MLV, and hMA-MLV with LCMV GP. Supernatant was collected for western blotting and the infectivity. Gag expression and viral release of HIV-1, MLV, and hMA-MLV are probed with capsid or HIV-1 MA antibody. GAPDH is probed for loading control of cell lysates. Average and standard deviation of infectious units produced from 4 independent experiments is also shown. 
Figure 4.5: MLV release is restricted in HeLa $\Delta$ Tetherin cells.

A.

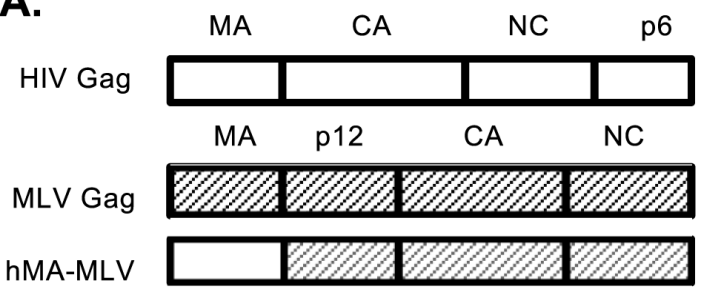

B.

\begin{tabular}{llll} 
Cells & FT & HeLa $\Delta$ Tetherin \\
\cline { 3 - 4 } & HIV MLV hMA-MLV & HIV MLV hMA-MLV
\end{tabular}

Cell lysates

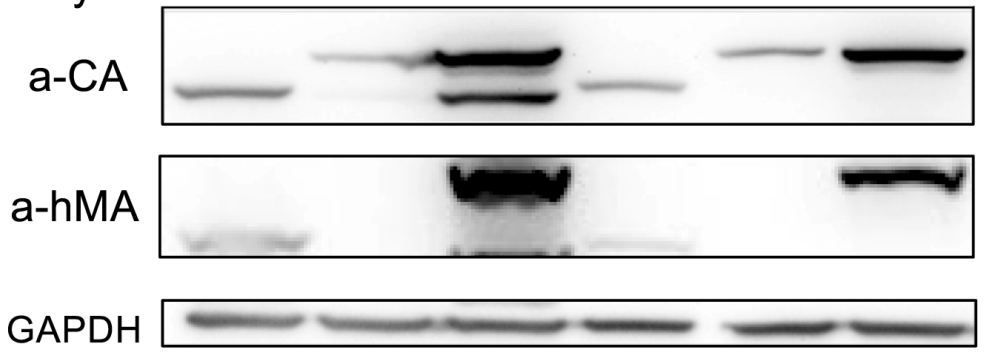

Viral release

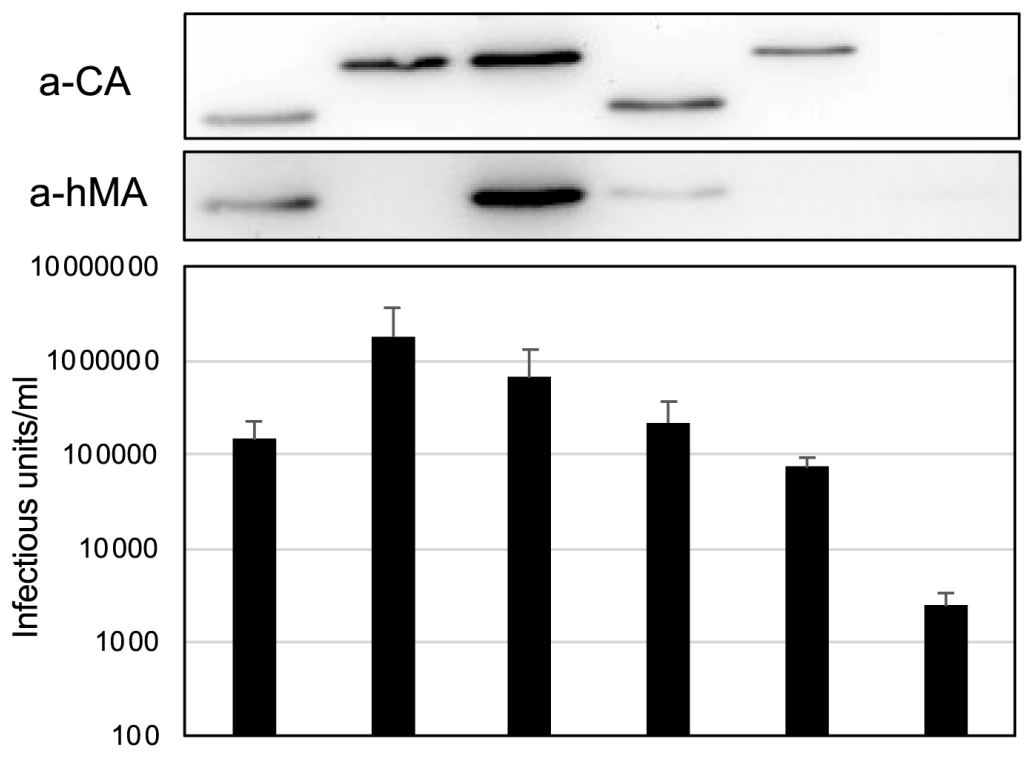


Figure 4.6: ZAP, TRIM25, and TRIM15 do not contribute to MLV restriction in HeLa cells.

(A) Confirmation of additional CRISPR knockout in HeLa $\Delta$ Tetherin cells. Sequencing result from PCR amplification including the regions of guide RNA is shown for both wildtype and clonal knockout cells. (B) 293FT cells, HeLa $\Delta$ Tetherin cells, and three double knockout HeLa cells were transfected with HIV-1 and hMA-MLV with LCMV GP. Supernatant was collected for western blotting and the infectivity. Gag expression and viral release of HIV-1 and hMA-MLV are blotted with HIV-1 MA antibody. GAPDH is probed for loading control in cell lysates. Representative image is shown. (C) Average and standard deviation of infectious units per $\mathrm{ml}$ from 5 independent experiments is shown. 
Figure 4.6: ZAP, TRIM25, and TRIM15 do not contribute to MLV restriction in HeLa cells.

A.

ZAP

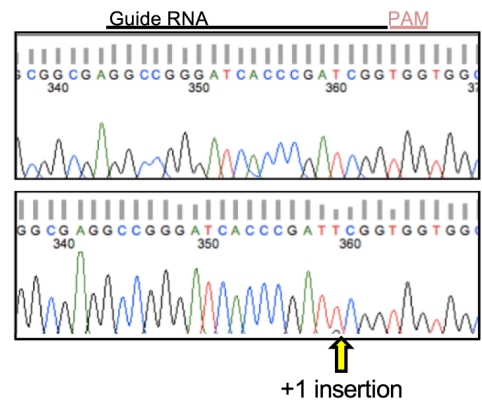

TRIM25

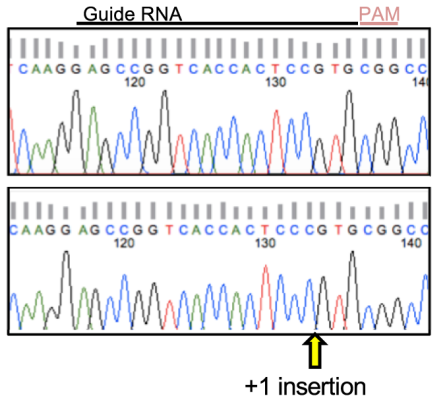

TRIM15
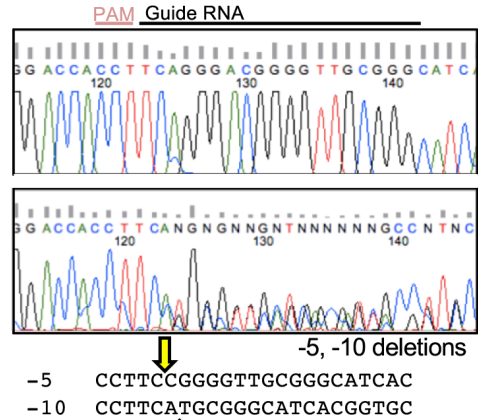

$\hat{\imath}$

B.

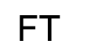
HeLa $\Delta$ Tetherin

ZAP

TRIM25

TRIM15

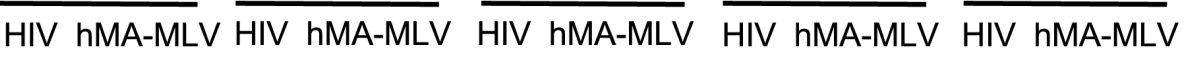
Cell lysates

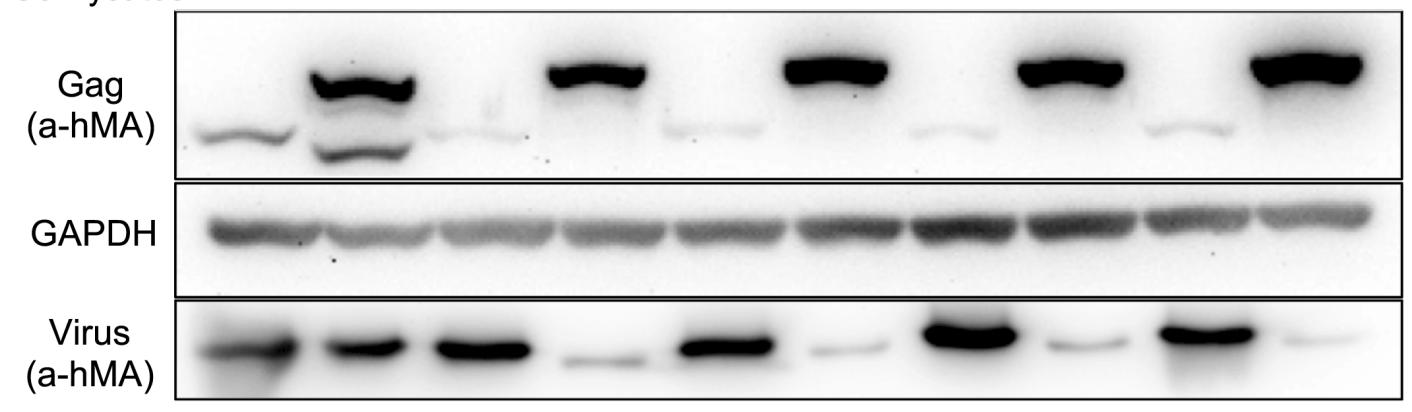

C.

- HIV-1 घhMA-MLV

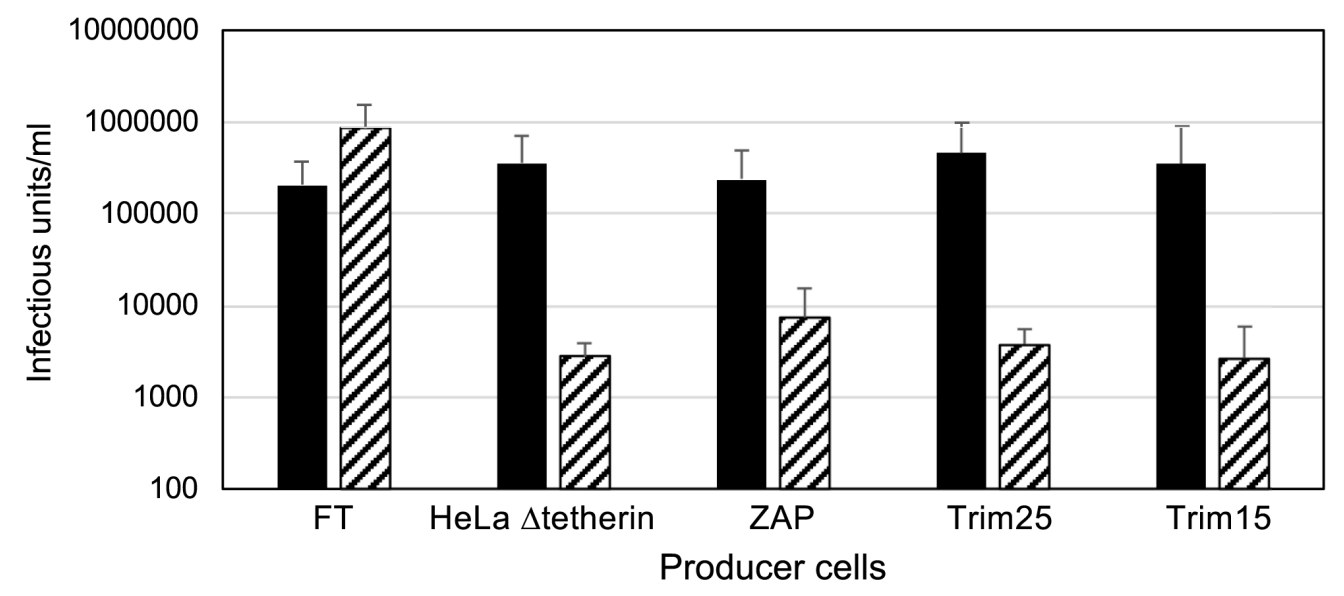


Figure 4.7: Immunofluorescence microscopy.

HIV-1 and hMA-MLV expression in 293FT cells (A) and HeLa $\Delta$ tetherin cells (B) are stained with anti-HIV-1 MA antibody and conducted on confocal microscopy.

Representative images are shown. GFP indicates transfection, and Alexa 555 indicates Gag proteins. 
Figure 4.7: Immunofluorescence microscopy.

\section{A. 293FT cells}

GFP

Alexa 555

Nucleus

Merge

HIV-1

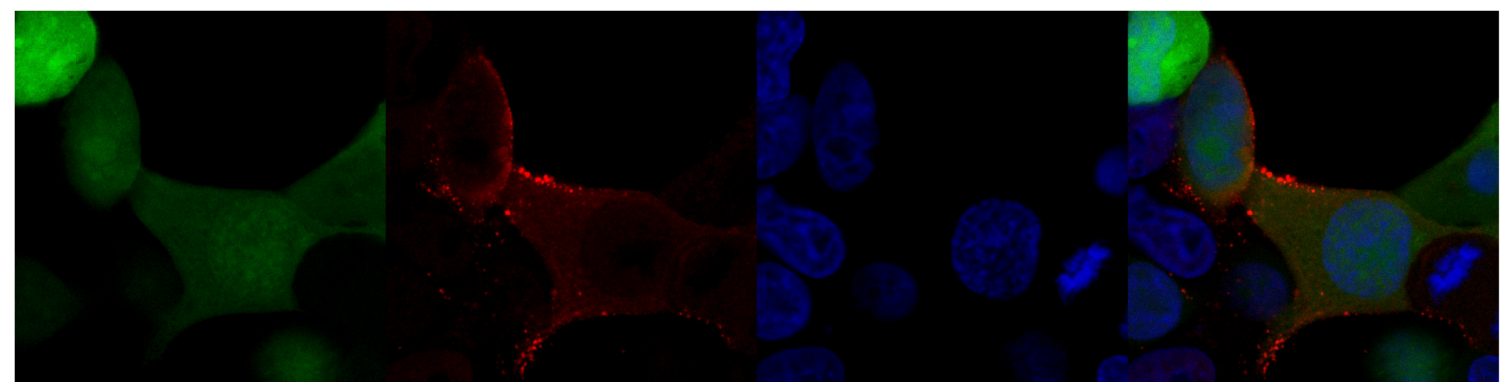

hMA-

MLV

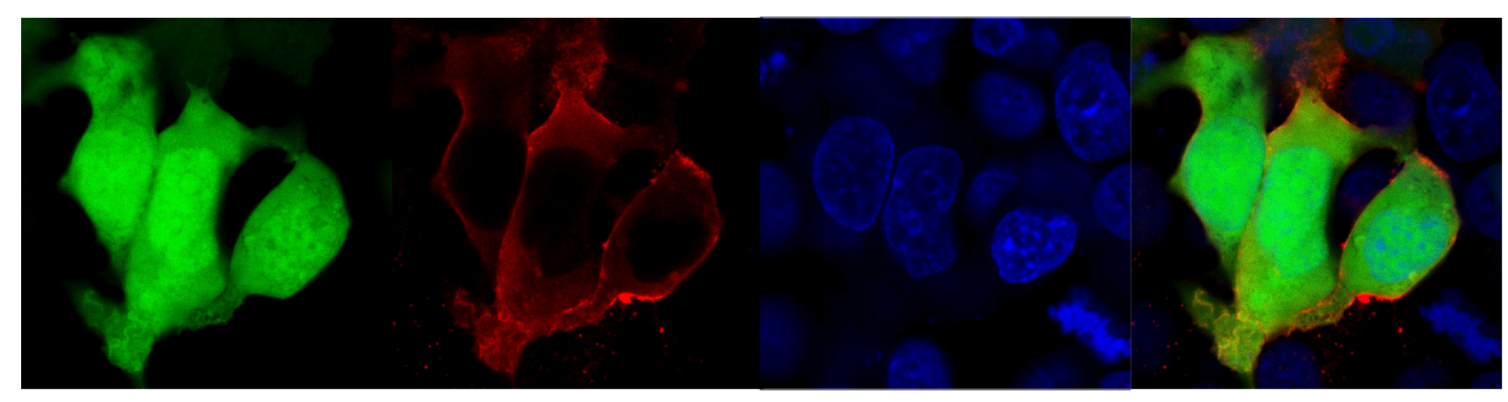

\section{B. HeLa $\Delta$ Tetherin}

HIV-1

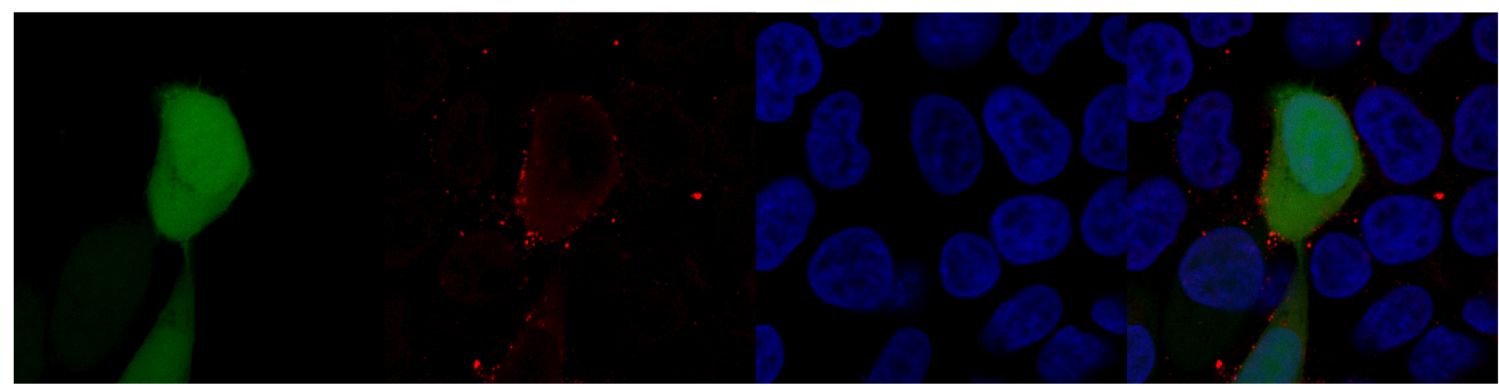

hMA-

MLV

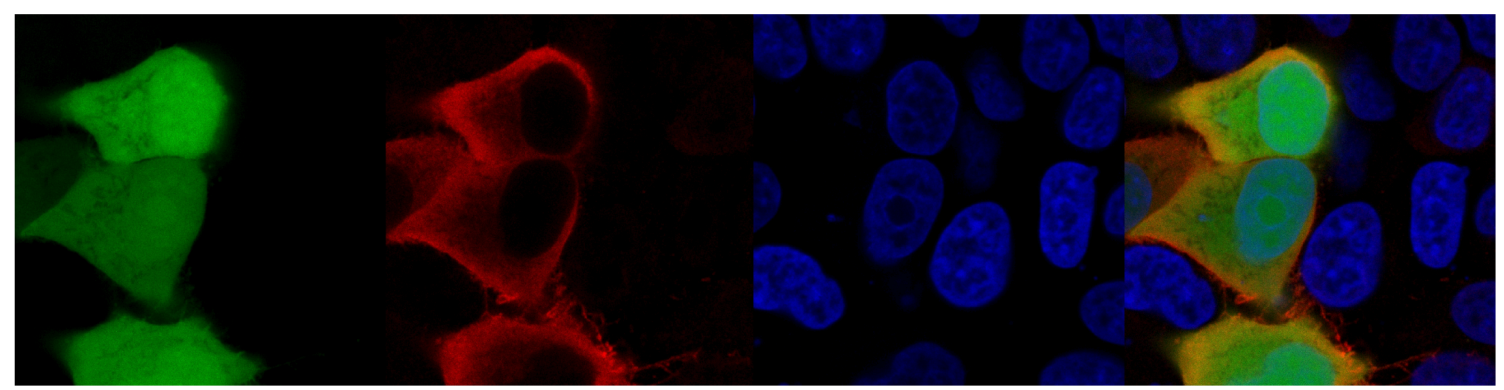


Figure 4.8: Syncytium induction assay.

Cells transfected with HIV-1 or hMA-MLV were co-cultured with cells transfected with VSV-G and pH-shocked with pH6 DMEM. After 24 hours, the supernatant was collected and transferred to target cells. Average and standard deviation of infectious units per $\mathrm{ml}$ is shown from 3 independent experiments. 
Figure 4.8: Syncytium induction assay.

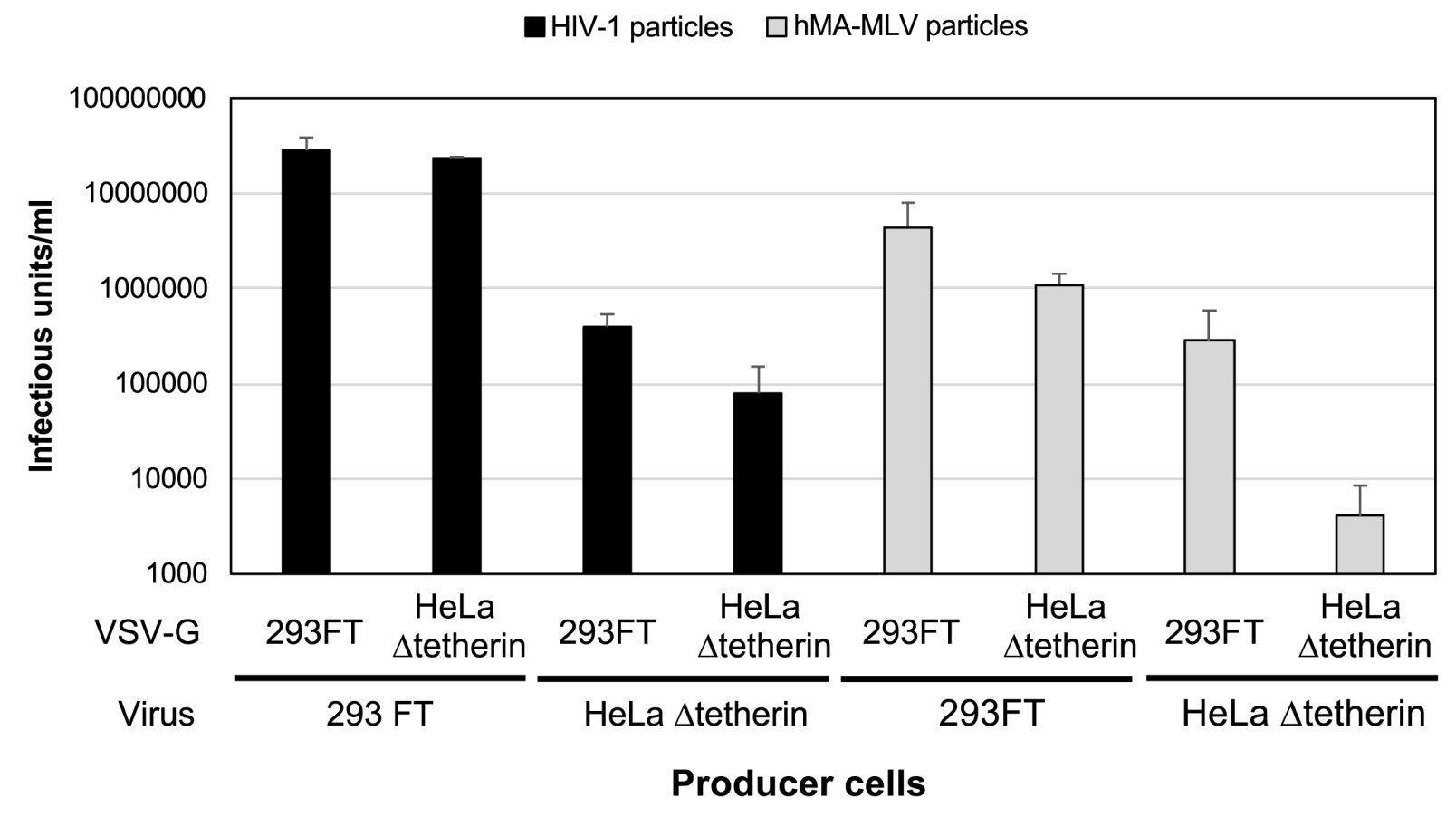




\section{V.Summary and Discussion}

Retroviruses have been studied in depth not only because of their malicious pathogenesis but also their potentiality as desirable gene therapy vectors due to the stable transgene expression. Retroviruses are capable of pseudotyping diverse glycoproteins from closely related or unrelated viral families, and this phenotype aids retroviral vectors to target specific cell types. Both gammaretroviral vectors and lentiviral vectors have been used in clinical trials and are expected to have a higher success rate as the vectors improve (181-183). Understanding the mechanism of glycoprotein acquisition will also contribute to improve retroviral vectors. My work shown here focuses on various factors that affect retroviral assembly, glycoprotein acquisition, and the fusogenicity of glycoprotein after budding.

Previous work shows that the HIV-1 accessory protein Vpu modulates GaLV Env and restrictes HIV-1 pseudotyping GaLV Env $(50,51)$. The mechanism of this modulation is similar to the Vpu modulation of $\mathrm{CD} 4$ and tetherin. As in $\mathrm{CD} 4$ degradation, the $\mathrm{S}^{52,56}$ residues in Vpu is essential to target GaLV Env, and also the lysine residues in GaLV Env $\mathrm{CT}$ are critical, which resembles the $\mathrm{CD} 4 \mathrm{CT}(51,56)$. As in BST-2/tetherin downmodulation, GaLV Env targeting is shown to be post-ER $(50,56)$. The $\mathrm{S}^{52,56}$ residues of $\mathrm{Vpu}$ are shown to be essential in modulation of all targets, but the co-factor requirement has been debated for BST-2/tetherin down-modulation $(54,74,75)$.

Our study presented here recapitulates the degradation of the mature form of GaLV Env (gp70) in a lysosome-dependent pathway, but blocking lysosomal degradation using inhibitors does not restore the infectivity. Further study shows that the surface expression of GaLV Env is reduced in the presence of Vpu. Infectivity could be restored by blocking 
polyubiquitination, supporting the importance of a ubiquitin ligase complex in the modulation of GaLV Env. Our novel CRISPR-Cas9 induced gene knockout study confirms that the co-factor $\beta \operatorname{TrCP}$ is required in the modulation of Vpu targets, $\mathrm{CD} 4$, GaLV Env, and BST-2/tetherin. Add back experiments to re-introduce $\beta \operatorname{TrCP}-2$ even further confirm the restored the activity of Vpu against these targets.

The study on virus-specific Env compatibility focuses more on the molecular mechanism of Env functionality dependent on Env CT. We find the GaLV Env remains less compatible with HIV-1 even in the absence of $\mathrm{Vpu}$, and the point mutation I170P in the CT makes the Env incompatibility with MLV particles. We narrow down the residues in the GaLV Env CT responsible for HIV-1 incompatibility and find that the defect was related to poor CT cleavage. The I170P point mutation in the GaLV Env CT is more striking in that it is almost completely non-functional with MLV particles. Further study shows that it is an outcome of a mixture of different factors. We hypothesize that three factors are involved in this phenotype.

First, I170P Env incorporation is restricted in MLV particles but not in HIV-1 particles, suggesting that the structure of Env trimer does not fit into the MLV MA lattice. Restoration of Env incorporation with the MA chimera or Env CT truncation supports this hypothesis. The second factor is that the I170P mutation enhanced Env fusogenicity with HIV-1 when R-peptide in intact. This made it appear that I170P is not defective in HIV-1 particles; however, when the R-peptide is removed, the I170P defect became more apparent even with HIV-1 particles. The third factor is related to virus-specific Env fusogenicity. Because MLV exhibited a more significant defect with the I170P mutation, a virus-specific factor is involved in the Env functionality, and we hypothesize that it is related to the lipid 
membrane environment. MLV Gag has the p12 domain between MA and CA, which could contribute to the different lipid environment.

Finally, we present evidence of cellular factors involved in the retroviral particle production. Even though various glycoproteins were reported to pseudotype with retroviruses, it has been unknown which glycoprotein is more compatible in different settings- such as different viruses or producer cells. We find that glycoproteins from 10 different viruses exhibit different compatibility from two different cell lines- 293FT and HeLa, and it is dependent on the different expression level of Env in most cases. RSV Env is an exception in that it does not successfully produce infectious particles either with HIV1 or MLV in HeLa cells. A striking finding is that the MLV infectious particle production is severely restricted in HeLa cells even in the absence of BST-2/tetherin restriction. Further study confirms that MLV assembly and budding is restricted in HeLa cells. Fusion with 293FT cells significantly enhances the infectivity, which supports the theory that a necessary host factor is not present in HeLa cells. We also conduct additional knockout studies to remove host factors that are known to possess antiviral activity against MLV. However, none of the additional knockout restore infectivity. 


\section{Future Direction}

The body of work described in this dissertation provides a basis of further studies. In depth study on the mechanism of Vpu activity leads us to develop a useful gain-offunction assay utilizing GaLV Env to screen small molecule inhibitors that target Vpu. Vpu modulation of CD4 and BST-2/tetherin does not only enhance viral release but also protects infected cells from Antibody Dependent Cell-mediated Cytotoxicity (ADCC) $(184,185)$. Currently there is no inhibitor that is known to target $\mathrm{Vpu}$, and the assay that involves GaLV Env is a novel tool to identify antiviral drugs that specifically target Vpu.

The study that focuses on the Env CT suggests an involvement of lipid-protein interaction during entry. The membrane binding property of the MA domain may affect the lipid environment of a virion, and further study is required to characterize how it affects Env fusogenicity. Understanding of this compatibility will help engineering a more efficient retroviral vector. In addition, the structure and role of Env CT still remains largely unknown, and our study provides a new insight on the Env fusogenicity in a virus-specific way.

The study on the cellular factors involved in MLV assembly provides a basis for a substantial further study. It is currently not known which necessary host factor is missing in HeLa cells, that is required for MLV assembly but not HIV-1. Extensive study to screen the target by reintroducing 293FT cDNA library may identify the novel cellular protein or a cellular pathway that is utilized by MLV. This will provide a new insight on an RNAprotein interaction or a protein-protein interaction in MLV assembly and budding. 


\section{Literature Cited}

1. Vogt PK. 1997. Historical introduction to the general properties of Retroviruses. In Coffin JM, Hughes SH, Varmus HE (ed), Retroviruses. Cold Spring Harbor Laboratory Press, New York.

2. Zhang W, Cao S, Martin JL, Mueller JD, Mansky LM. 2015. Morphology and ultrastructure of retrovirus particles. AIMS Biophys 2:343-369.

3. Goff SP. 2007. Retroviridae: The retroviruses and their replication. In Knipe DM, Howley PM (ed), Fields' Virology, vol 2. Lippincott Williams \& Wilkins, Philadelphia.

4. Vogt VM. 1997. Retroviral virions and genomes. In Coffin JM, Hughes SH, Varmus HE (ed), Retroviruses. Cold Spring Harbor Laboratory Press, New York.

5. Mertz JA, Simper MS, Lozano MM, Payne SM, Dudley JP. 2005. Mouse mammary tumor virus encodes a self-regulatory RNA export protein and is a complex retrovirus. J Virol 79:14737-47.

6. Hofacre A, Nitta T, Fan H. 2009. Jaagsiekte sheep retrovirus encodes a regulatory factor, Rej, required for synthesis of Gag protein. J Virol 83:12483-98.

7. Pizzato M. 2010. MLV glycosylated-Gag is an infectivity factor that rescues Nefdeficient HIV-1. Proc Natl Acad Sci U S A 107:9364-9.

8. Weiss RA. 2006. The discovery of endogenous retroviruses. Retrovirology 3:67.

9. Rabson AB, Graves BJ. 1997. Synthesis and processing of viral RNA. In Coffin JM, Hughes SH, Varmus HE (ed), Retroviruses. Cold Spring Harbor Laboratory Press, New York. 
10. Freed EO, Martin MA. 2007. HIVs and their replication. In Knipe DM, Howley PM (ed), Fields' Virology, vol 2. Lippincott Williams \& Wilkins, Philadelphia.

11. Keane SC, Summers MF. 2016. NMR Studies of the Structure and Function of the HIV-1 5'-Leader. Viruses 8.

12. Le Sage V, Mouland AJ, Valiente-Echeverria F. 2014. Roles of HIV-1 capsid in viral replication and immune evasion. Virus Res 193:116-29.

13. Rene B, Mauffret O, Fosse P. 2018. Retroviral nucleocapsid proteins and DNA strand transfers. Biochim Open 7:10-25.

14. Votteler J, Sundquist WI. 2013. Virus budding and the ESCRT pathway. Cell Host Microbe 14:232-41.

15. Henzy JE, Coffin JM. 2013. Betaretroviral envelope subunits are noncovalently associated and restricted to the mammalian class. J Virol 87:1937-46.

16. Neil SJ, Zang T, Bieniasz PD. 2008. Tetherin inhibits retrovirus release and is antagonized by HIV-1 Vpu. Nature 451:425-30.

17. Rosa A, Chande A, Ziglio S, De Sanctis V, Bertorelli R, Goh SL, McCauley SM, Nowosielska A, Antonarakis SE, Luban J, Santoni FA, Pizzato M. 2015. HIV-1 Nef promotes infection by excluding SERINC5 from virion incorporation. Nature $526: 212-7$.

18. Usami Y, Wu Y, Gottlinger HG. 2015. SERINC3 and SERINC5 restrict HIV-1 infectivity and are counteracted by Nef. Nature 526:218-23.

19. Sheehy AM, Gaddis NC, Malim MH. 2003. The antiretroviral enzyme APOBEC3G is degraded by the proteasome in response to HIV-1 Vif. Nat Med 9:1404-7.

20. Ahi YS, Zhang S, Thappeta Y, Denman A, Feizpour A, Gummuluru S, Reinhard B, Muriaux D, Fivash MJ, Rein A. 2016. Functional interplay between Murine 
Leukemia Virus Glycogag, Serinc5, and surface glycoprotein governs virus entry, with opposite effects on gammaretroviral and Ebolavirus glycoproteins. MBio 7.

21. Telesnitsky A, Goff SP. 1997. Reverse transcriptase and the generation of retroviral DNA. In Coffin JM, Hughes SH, Varmus HE (ed), Retroviruses. Cold Spring Harbor Laboratory Press, New York.

22. Brown PO. 1997. Integration. In Coffin JM, Hughes SH, Varmus HE (ed), Retroviruses. Cold Spring Harbor Laboratory Press, New York.

23. Cullen BR. 2003. Nuclear mRNA export: insights from virology. Trends in Biochemical Sciences 28:419-424.

24. Bodem J, Schied T, Gabriel R, Rammling M, Rethwilm A. 2011. Foamy virus nuclear RNA export is distinct from that of other retroviruses. J Virol 85:2333-41.

25. Johnson MC. 2011. Mechanisms for Env glycoprotein acquisition by retroviruses. AIDS Res Hum Retroviruses 27:239-47.

26. Murakami T. 2012. Retroviral Env glycoprotein trafficking and incorporation into virions. Mol Biol Int 2012:682850.

27. Mercenne G, Alam SL, Arii J, Lalonde MS, Sundquist WI. 2015. Angiomotin functions in HIV-1 assembly and budding. Elife 4 .

28. Patnaik A, Chau V, Wills JW. 2000. Ubiquitin is part of the retrovirus budding machinery. Proc Natl Acad Sci U S A 97:13069-13074.

29. Zhadina M, McClure MO, Johnson MC, Bieniasz PD. 2007. Ubiquitin-dependent virus particle budding without viral protein ubiquitination. Proc Natl Acad Sci U S A 104:20031-6. 
30. Loving R, Wu SR, Sjoberg M, Lindqvist B, Garoff H. 2012. Maturation cleavage of the Murine Leukemia Virus Env precursor separates the transmembrane subunits to prime it for receptor triggering. Proc Natl Acad Sci U S A 109:7735-7740.

31. Granoff A, Hirst GK. 1954. Experimental production of combination forms of virus. IV. Mixed influenza A-Newcastle disease virus infections. Proc Soc Exp Biol Med $86: 84-88$.

32. Zavada J. 1982. The pseudotypic paradox. J Gen Virol 63:15-24.

33. Burns JC, Friedmann T, Driever W, Burrascano M, Yee JK. 1993. Vesicular stomatitis virus $\mathrm{G}$ glycoprotein pseudotyped retroviral vectors: concentration to very high titer and efficient gene transfer into mammalian and nonmammalian cells. Proc Natl Acad Sci U S A 90:8033-8037.

34. Kumar M, Bradow BP, Zimmerberg J. 2003. Large-scale production of pseudotyped lentiviral vectors using baculovirus GP64. Hum Gene Ther 14:67-77.

35. Sena-Esteves M, Tebbets JC, Steffens S, Crombleholme T, Flake AW. 2004. Optimized large-scale production of high titer lentivirus vector pseudotypes. J Virol Methods 122:131-9.

36. Hu D, Zhang J, Wang H, Liu S, Yu L, Sun L, Qu Y. 2014. Chikungunya virus glycoproteins pseudotype with lentiviral vectors and reveal a broad spectrum of cellular tropism. PLoS One 9:e110893.

37. Sinn PL, Coffin JE, Ayithan N, Holt KH, Maury W. 2017. Lentiviral Vectors Pseudotyped with Filoviral Glycoproteins. Methods Mol Biol 1628:65-78.

38. Tedbury PR, Freed EO. 2015. The cytoplasmic tail of retroviral envelope glycoproteins. Prog Mol Biol Transl Sci 129:253-84. 
39. Murakami T, Freed EO. 2000. The long cytoplasmic tail of gp41 is required in a cell type-dependent manner for HIV-1 envelope glycoprotein incorporation into virions. Proc Natl Acad Sci U S A 97:343-348.

40. Qi M, Chu H, Chen X, Choi J, Wen X, Hammonds J, Ding L, Hunter E, Spearman P. 2015. A tyrosine-based motif in the HIV-1 envelope glycoprotein tail mediates cell-type- and Rab11-FIP1C-dependent incorporation into virions. Proc Natl Acad Sci U S A 112:7575-80.

41. Jin J, Li F, Mothes W. 2011. Viral determinants of polarized assembly for the murine leukemia virus. J Virol 85:7672-82.

42. Lucas TM, Lyddon TD, Grosse SA, Johnson MC. 2010. Two distinct mechanisms regulate recruitment of Murine Leukemia Virus envelope protein to retroviral assembly sites. Virology 405:548-55.

43. Jouvenet N, Neil SJ, Zhadina M, Zang T, Kratovac Z, Lee Y, McNatt M, Hatziioannou T, Bieniasz PD. 2009. Broad-spectrum inhibition of retroviral and filoviral particle release by tetherin. J Virol 83:1837-44.

44. Qi M, Williams JA, Chu H, Chen X, Wang JJ, Ding L, Akhirome E, Wen X, Lapierre LA, Goldenring JR, Spearman P. 2013. Rab11-FIP1C and Rab14 direct plasma membrane sorting and particle incorporation of the HIV-1 envelope glycoprotein complex. PLoS Pathog 9:e1003278.

45. Ohno H, Aguilar RC, Fournier M-C, Hennecke S, Cosson P, Bonifacino JS. 1997. Interaction of endocytic signals from the HIV-1 Envelope glycoprotein complex with members of the adaptor medium chain family. Virology 238:305-315.

46. Lodge R, Delamarre L, Lalonde JP, Alvarado J, Sanders DA, Dokhélar MC, Cohen EA, Lemay G. 1997. Two distinct oncornaviruses harbor an intracytoplasmic 
tyrosine-based basolateral targeting signal in their viral envelope glycoprotein. J Virol 71:5696-5702.

47. Berlioz-Torrent C, Shacklett BL, Erdtmann L, Delamarre L, Bouchaert I, Sonigo P, Dokhelar MC, Benarous R. 1999. Interactions of the cytoplasmic domains of human and simian retroviral transmembrane proteins with components of the clathrin adaptor complexes modulate intracellular and cell surface expression of envelope glycoproteins. J Virol 73:1350-1361.

48. Blot V, Lopez-Verges S, Breton M, Pique C, Berlioz-Torrent C, Grange MP. 2006. The conserved dileucine- and tyrosine-based motifs in MLV and MPMV envelope glycoproteins are both important to regulate a common Env intracellular trafficking. Retrovirology $3: 62$.

49. Breed MW, Elser SE, Torben W, Jordan AP, Aye PP, Midkiff C, Schiro F, Sugimoto C, Alvarez-Hernandez X, Blair RV, Somasunderam A, Utay NS, Kuroda MJ, Pahar B, Wiseman RW, O'Connor DH, LaBranche CC, Montefiori DC, Marsh M, Li Y, Piatak M, Jr., Lifson JD, Keele BF, Fultz PN, Lackner AA, Hoxie JA. 2015. Elite Control, Gut CD4 T Cell Sparing, and Enhanced Mucosal T Cell Responses in Macaca nemestrina Infected by a Simian Immunodeficiency Virus Lacking a gp41 Trafficking Motif. J Virol 89:10156-75.

50. Christodoulopoulos I, Droniou-Bonzom ME, Oldenburg JE, Cannon PM. 2010. Vpu-dependent block to incorporation of GaLV Env into lentiviral vectors. Retrovirology 7:4.

51. Lucas TM, Lyddon TD, Cannon PM, Johnson MC. 2010. Pseudotyping incompatibility between HIV-1 and Gibbon Ape Leukemia Virus Env is modulated by Vpu. J Virol 84:2666-74. 
52. Magadan JG, Perez-Victoria FJ, Sougrat R, Ye Y, Strebel K, Bonifacino JS. 2010. Multilayered mechanism of CD4 downregulation by HIV-1 Vpu involving distinct ER retention and ERAD targeting steps. PLoS Pathog 6:e1000869.

53. McNatt MW, Zang T, Hatziioannou T, Bartlett M, Fofana IB, Johnson WE, Neil SJ, Bieniasz PD. 2009. Species-specific activity of HIV-1 Vpu and positive selection of tetherin transmembrane domain variants. PLoS Pathog 5:e1000300.

54. Mitchell RS, Katsura C, Skasko MA, Fitzpatrick K, Lau D, Ruiz A, Stephens EB, Margottin-Goguet F, Benarous R, Guatelli JC. 2009. Vpu antagonizes BST-2mediated restriction of $\mathrm{HIV}-1$ release via beta-TrCP and endo-lysosomal trafficking. PLoS Pathog 5:e1000450.

55. Miyagi E, Andrew AJ, Kao S, Strebel K. 2009. Vpu enhances HIV-1 virus release in the absence of Bst-2 cell surface down-modulation and intracellular depletion. Proc Natl Acad Sci U S A 106:2868-73.

56. Janaka SK, Lucas TM, Johnson MC. 2011. Sequences in Gibbon Ape Leukemia Virus envelope that confer sensitivity to HIV-1 accessory protein Vpu. Journal of Virology 85:11945-11954.

57. Lucas TM, Janaka SK, Stephens EB, Johnson MC. 2012. Vpu downmodulates two distinct targets, tetherin and gibbon ape leukemia virus envelope, through shared features in the Vpu cytoplasmic tail. PLoS One 7:e51741.

58. Song YE, Cyburt D, Lucas TM, Gregory DA, Lyddon TD, Johnson MC. 2018. betaTrCP is Required for HIV-1 Vpu Modulation of CD4, GaLV Env, and BST2/Tetherin. Viruses 10. 
59. Sugden SM, Bego MG, Pham TN, Cohen EA. 2016. Remodeling of the Host Cell Plasma Membrane by HIV-1 Nef and Vpu: A Strategy to Ensure Viral Fitness and Persistence. Viruses 8:67.

60. Andrew A, Strebel K. 2010. HIV-1 Vpu targets cell surface markers CD4 and BST2 through distinct mechanisms. Mol Aspects Med 31:407-17.

61. Willey RL, Maldarelli F, Martin MA, Strebel K. 1992. Human immunodeficiency virus type 1 Vpu protein induces rapid degradation of CD4. J Virol 66:7193-200.

62. Van Damme N, Goff D, Katsura C, Jorgenson RL, Mitchell R, Johnson MC, Stephens EB, Guatelli J. 2008. The interferon-induced protein BST-2 restricts HIV1 release and is downregulated from the cell surface by the viral Vpu protein. Cell Host Microbe 3:245-52.

63. Schubert U, Schneider T, Henklein P, Hoffmann K, Berthold E, Hauser H, Pauli G, Porstmann T. 1992. Human-immunodeficiency-virus-type-1-encoded Vpu protein is phosphorylated by casein kinase II. Eur J Biochem 204:875-83.

64. Chen MY, Maldarelli F, Karczewski MK, Willey RL, Strebel K. 1993. Human immunodeficiency virus type $1 \mathrm{Vpu}$ protein induces degradation of CD4 in vitro: the cytoplasmic domain of CD4 contributes to Vpu sensitivity. Journal of Virology $67: 3877-84$.

65. Schubert U, Strebel K. 1994. Differential activities of the human immunodeficiency virus type 1-encoded Vpu protein are regulated by phosphorylation and occur in different cellular compartments. Journal of Virology 68:2260-71. 
66. Schubert U, Bour S, Ferrer-Montiel AV, Montal M, Maldarell F, Strebel K. 1996. The two biological activities of human immunodeficiency virus type 1 Vpu protein involve two separable structural domains. Journal of Virology 70:809-19.

67. Schubert U, Anton LC, Bacik I, Cox JH, Bour S, Bennink JR, Orlowski M, Strebel K, Yewdell JW. 1998. CD4 glycoprotein degradation induced by human immunodeficiency virus type $1 \mathrm{Vpu}$ protein requires the function of proteasomes and the ubiquitin-conjugating pathway. Journal of Virology 72:2280-8.

68. Margottin F, Bour SP, Durand H, Selig L, Benichou S, Richard V, Thomas D, Strebel K, Benarous R. 1998. A novel human WD protein, h-beta TrCp, that interacts with HIV-1 Vpu connects CD4 to the ER degradation pathway through an F-box motif. Molecular Cell 1:565-74.

69. Butticaz C, Michielin O, Wyniger J, Telenti A, Rothenberger S. 2007. Silencing of both beta-TrCP1 and HOS (beta-TrCP2) is required to suppress human immunodeficiency virus type 1 Vpu-mediated CD4 down-modulation. J Virol $81: 1502-5$.

70. Dube M, Roy BB, Guiot-Guillain P, Binette J, Mercier J, Chiasson A, Cohen EA. 2010. Antagonism of tetherin restriction of HIV-1 release by Vpu involves binding and sequestration of the restriction factor in a perinuclear compartment. PLoS Pathog 6:e1000856.

71. Andrew AJ, Miyagi E, Strebel K. 2011. Differential effects of human immunodeficiency virus type $1 \mathrm{Vpu}$ on the stability of BST-2/tetherin. J Virol 85:2611-9. 
72. Schmidt S, Fritz JV, Bitzegeio J, Fackler OT, Keppler OT. 2011. HIV-1 Vpu blocks recycling and biosynthetic transport of the intrinsic immunity factor CD317/tetherin to overcome the virion release restriction. MBio 2:e00036-11.

73. Douglas JL, Viswanathan K, McCarroll MN, Gustin JK, Fruh K, Moses AV. 2009. Vpu directs the degradation of the human immunodeficiency virus restriction factor BST-2/Tetherin via a \{beta\}TrCP-dependent mechanism. J Virol 83:7931-47.

74. Tervo HM, Homann S, Ambiel I, Fritz JV, Fackler OT, Keppler OT. 2011. betaTrCP is dispensable for Vpu's ability to overcome the CD317/Tetherin-imposed restriction to HIV-1 release. Retrovirology 8:9.

75. Kueck T, Foster TL, Weinelt J, Sumner JC, Pickering S, Neil SJ. 2015. Serine Phosphorylation of HIV-1 Vpu and Its Binding to Tetherin Regulates Interaction with Clathrin Adaptors. PLoS Pathog 11:e1005141.

76. Pujol FM, Laketa V, Schmidt F, Mukenhirn M, Muller B, Boulant S, Grimm D, Keppler OT, Fackler OT. 2016. HIV-1 Vpu Antagonizes CD317/Tetherin by Adaptor Protein-1-Mediated Exclusion from Virus Assembly Sites. J Virol 90:6709-23.

77. Mangeat B, Gers-Huber G, Lehmann M, Zufferey M, Luban J, Piguet V. 2009. HIV-1 Vpu neutralizes the antiviral factor Tetherin/BST-2 by binding it and directing its beta-TrCP2-dependent degradation. PLoS Pathog 5:e1000574.

78. Iwabu Y, Fujita H, Kinomoto M, Kaneko K, Ishizaka Y, Tanaka Y, Sata T, Tokunaga K. 2009. HIV-1 accessory protein Vpu internalizes cell-surface BST2/tetherin through transmembrane interactions leading to lysosomes. J Biol Chem 284:35060-72. 
79. Stoneham CA, Singh R, Jia X, Xiong Y, Guatelli J. 2017. Endocytic activity of HIV-1 Vpu: Phosphoserine-dependent interactions with clathrin adaptors. Traffic 18:545-561.

80. Ramirez PW, DePaula-Silva AB, Szaniawski M, Barker E, Bosque A, Planelles V. 2015. HIV-1 Vpu utilizes both cullin-RING ligase (CRL) dependent and independent mechanisms to downmodulate host proteins. Retrovirology 12:65.

81. Tokarev A, Stoneham C, Lewinski MK, Mukim A, Deshmukh S, Vollbrecht T, Spina CA, Guatelli J. 2016. Pharmacologic Inhibition of Nedd8 Activation Enzyme Exposes CD4-Induced Epitopes within Env on Cells Expressing HIV-1. J Virol 90:2486-502.

82. Goffinet C, Allespach I, Homann S, Tervo HM, Habermann A, Rupp D, Oberbremer L, Kern C, Tibroni N, Welsch S, Krijnse-Locker J, Banting G, Krausslich HG, Fackler OT, Keppler OT. 2009. HIV-1 antagonism of CD317 is species specific and involves Vpu-mediated proteasomal degradation of the restriction factor. Cell Host Microbe 5:285-97.

83. Janvier K, Pelchen-Matthews A, Renaud JB, Caillet M, Marsh M, Berlioz-Torrent C. 2011. The ESCRT-0 component HRS is required for HIV-1 Vpu-mediated BST2/tetherin down-regulation. PLoS Pathog 7:e1001265.

84. Dube M, Paquay C, Roy BB, Bego MG, Mercier J, Cohen EA. 2011. HIV-1 Vpu antagonizes BST-2 by interfering mainly with the trafficking of newly synthesized BST-2 to the cell surface. Traffic 12:1714-29.

85. Gustin JK, Douglas JL, Bai Y, Moses AV. 2012. Ubiquitination of BST-2 protein by HIV-1 Vpu protein does not require lysine, serine, or threonine residues within the BST-2 cytoplasmic domain. J Biol Chem 287:14837-50. 
86. Kueck T, Neil SJ. 2012. A cytoplasmic tail determinant in HIV-1 Vpu mediates targeting of tetherin for endosomal degradation and counteracts interferon-induced restriction. PLoS Pathog 8:e1002609.

87. Waheed AA, Kuruppu ND, Felton KL, D'Souza D, Freed EO. 2014. In COS cells Vpu can both stabilize tetherin expression and counteract its antiviral activity. PLoS One 9:e111628.

88. Janaka SK, Faurot J, Johnson MC. 2013. Functional complementation of a model target to study Vpu sensitivity. PLOS One 8(6): e68507.

89. Sherer NM, Lehmann MJ, Jimenez-Soto LF, Ingmundson A, Horner SM, Cicchetti G, Allen PG, Pypaert M, Cunningham JM, Mothes W. 2003. Visualization of retroviral replication in living cells reveals budding into multivesicular bodies. Traffic 4:785-801.

90. Ran FA, Hsu PD, Wright J, Agarwala V, Scott DA, Zhang F. 2013. Genome engineering using the CRISPR-Cas9 system. Nat Protoc 8:2281-2308.

91. Gomez LM, Pacyniak E, Flick M, Hout DR, Gomez ML, Nerrienet E, Ayouba A, Santiago ML, Hahn BH, Stephens EB. 2005. Vpu-mediated CD4 down-regulation and degradation is conserved among highly divergent SIV(cpz) strains. Virology $335: 46-60$.

92. Emanuele MJ, Elia AE, Xu Q, Thoma CR, Izhar L, Leng Y, Guo A, Chen YN, Rush J, Hsu PW, Yen HC, Elledge SJ. 2011. Global identification of modular cullin-RING ligase substrates. Cell 147:459-74.

93. Schindler M, Rajan D, Banning C, Wimmer P, Koppensteiner H, Iwanski A, Specht A, Sauter D, Dobner T, Kirchhoff F. 2010. Vpu serine 52 dependent counteraction 
of tetherin is required for HIV-1 replication in macrophages, but not in ex vivo human lymphoid tissue. Retrovirology 7:1.

94. Perez-Caballero D, Zang T, Ebrahimi A, McNatt MW, Gregory DA, Johnson MC, Bieniasz PD. 2009. Tetherin inhibits HIV-1 release by directly tethering virions to cells. Cell 139:499-511.

95. Lewis BC, Chinnasamy N, Morgan RA, Varmus HE. 2001. Development of an avian leukosis-sarcoma virus subgroup A pseudotyped lentiviral vector. J Virol 75:9339-44.

96. Boussif O, Lezoualc'h F, Zanta MA, Mergny MD, Scherman D, Demeneix B, Behr JP. 1995. A versatile vector for gene and oligonucleotide transfer into cells in culture and in vivo: polyethylenimine. Proc Natl Acad Sci U S A 92:7297-301.

97. Hallenberger S, Bosch V, Angliker H, Shaw E, Klenk HD, Garten W. 1992. Inhibition of furin-mediated cleavage activation of HIV-1 glycoprotein gp160. Nature 360:358-61.

98. Arnason T, Ellison MJ. 1994. Stress resistance in Saccharomyces cerevisiae is strongly correlated with assembly of a novel type of multiubiquitin chain. Mol Cell Biol 14:7876-83.

99. Hampe C, Ardila-Osorio H, Fournier M, Brice A, Corti O. 2006. Biochemical analysis of Parkinson's disease-causing variants of Parkin, an E3 ubiquitin-protein ligase with monoubiquitylation capacity. Hum Mol Genet 15:2059-75.

100. Lim KL, Chew KC, Tan JM, Wang C, Chung KK, Zhang Y, Tanaka Y, Smith W, Engelender S, Ross CA, Dawson VL, Dawson TM. 2005. Parkin mediates nonclassical, proteasomal-independent ubiquitination of synphilin-1: implications for Lewy body formation. J Neurosci 25:2002-9. 
101. Oshikawa K, Matsumoto M, Oyamada K, Nakayama KI. 2012. Proteome-wide identification of ubiquitylation sites by conjugation of engineered lysine-less ubiquitin. J Proteome Res 11:796-807.

102. Zhou R, Patel SV, Snyder PM. 2007. Nedd4-2 catalyzes ubiquitination and degradation of cell surface ENaC. J Biol Chem 282:20207-12.

103. Soucy TA, Smith PG, Milhollen MA, Berger AJ, Gavin JM, Adhikari S, Brownell JE, Burke KE, Cardin DP, Critchley S, Cullis CA, Doucette A, Garnsey JJ, Gaulin JL, Gershman RE, Lublinsky AR, McDonald A, Mizutani H, Narayanan U, Olhava EJ, Peluso S, Rezaei M, Sintchak MD, Talreja T, Thomas MP, Traore T, Vyskocil S, Weatherhead GS, Yu J, Zhang J, Dick LR, Claiborne CF, Rolfe M, Bolen JB, Langston SP. 2009. An inhibitor of NEDD8-activating enzyme as a new approach to treat cancer. Nature 458:732-6.

104. Merlet J, Burger J, Gomes JE, Pintard L. 2009. Regulation of cullin-RING E3 ubiquitin-ligases by neddylation and dimerization. Cell Mol Life Sci 66:1924-38.

105. McNatt MW, Zang T, Bieniasz PD. 2013. Vpu Binds Directly to Tetherin and Displaces It from Nascent Virions. PLoS Pathogens 9:e1003299.

106. Wang T, Birsoy K, Hughes NW, Krupczak KM, Post Y, Wei JJ, Lander ES, Sabatini DM. 2015. Identification and characterization of essential genes in the human genome. Science 350:1096-101.

107. Song YE, Olinger GY, Janaka SK, Johnson MC. 2019. Sequence determinants in gammaretroviral Env cytoplasmic tails dictate virus-specific pseudotyping compatibility. J Virol doi:10.1128/JVI.02172-18.

108. Harrison SC. 2015. Viral membrane fusion. Virology 479-480:498-507. 
109. Ragheb JA, Anderson WF. 1994. pH-independent Murine Leukemia Virus ecotropic envelope-mediated cell fusion: implications for the role of the R peptide and p12E TM in viral entry. J Virol 68:3220-3231.

110. Rein A, Mirro J, Haynes JG, Ernst SM, Nagashima K. 1994. Function of the cytoplasmic domain of a retroviral transmembrane protein: p15E-p2E cleavage activates the membrane fusion capability of the Murine Leukemia Virus Env protein. J Virol 68:1773-1781.

111. Brody BA, Rhee SS, Sommerfelt MA, Hunter E. 1992. A viral protease-mediated cleavage of the transmembrane glycoprotein of Mason-Pfizer monkey virus can be suppressed by mutations within the matrix protein. Proc Natl Acad Sci U S A 89:3443-3447.

112. Christodoulopoulos I, Cannon PM. 2001. Sequences in the cytoplasmic tail of the Gibbon Ape Leukemia Virus envelope protein that prevent its incorporation into lentivirus vectors. J Virol 75:4129-38.

113. Bobkova M, Stitz J, Engelstadter M, Cichutek K, Buchholz CJ. 2002. Identification of R-peptides in envelope proteins of C-type retroviruses. J Gen Virol 83:22412246.

114. Rice NR, Henderson LE, Sowder RC, Copeland TD, Oroszlan S, Edwards JF. 1990. Synthesis and processing of the transmembrane envelope protein of Equine Infectious Anemia Virus. J Virol 64:3770-3778.

115. Karshin WL, Arcement LJ, Naso RB, Arlinghaus RB. 1977. Common precursor for rauscher leukemia virus gp69/71, p15(E), and p12(E). J Virol 23:787-798. 
116. Jiang J, Aiken C. 2007. Maturation-dependent Human Immunodeficiency Virus type 1 particle fusion requires a carboxyl-terminal region of the gp41 cytoplasmic tail. J Virol 81:9999-10008.

117. Joyner AS, Willis JR, Crowe JE, Jr., Aiken C. 2011. Maturation-induced cloaking of neutralization epitopes on HIV-1 particles. PLoS Pathog 7:e1002234.

118. Wyma DJ, Jiang J, Shi J, Zhou J, Lineberger JE, Miller MD, Aiken C. 2004. Coupling of Human Immunodeficiency Virus type 1 fusion to virion maturation: a novel role of the gp41 cytoplasmic tail. Journal of Virology 78:3429-3435.

119. Santos da Silva E, Mulinge M, Perez Bercoff D. 2013. The frantic play of the concealed HIV envelope cytoplasmic tail. Retrovirology 10.

120. Postler TS, Desrosiers RC. 2013. The tale of the long tail: the cytoplasmic domain of HIV-1 gp41. J Virol 87:2-15.

121. Steckbeck JD, Kuhlmann AS, Montelaro RC. 2014. Structural and functional comparisons of retroviral envelope protein C-terminal domains: still much to learn. Viruses 6:284-300.

122. Spearman P. 2018. Viral interactions with host cell Rab GTPases. Small GTPases 9:192-201.

123. Stitz J, Buchholz CJ, Engelstadter M, Uckert W, Bloemer U, Schmitt I, Cichutek K. 2000. Lentiviral vectors pseudotyped with envelope glycoproteins derived from Gibbon Ape Leukemia Virus and Murine Leukemia Virus 10A1. Virology 273:1620.

124. Sandrin V, Boson B, Salmon P, Gay W, Negre D, Le Grand R, Trono D, Cosset FL. 2002. Lentiviral vectors pseudotyped with a modified RD114 envelope glycoprotein show increased stability in sera and augmented transduction of 
primary lymphocytes and CD34+ cells derived from human and nonhuman primates. Blood 100:823-32.

125. Sandrin V, Muriaux D, Darlix JL, Cosset FL. 2004. Intracellular trafficking of Gag and Env proteins and their interactions modulate pseudotyping of retroviruses. J Virol 78:7153-64.

126. Sandrin V, Cosset FL. 2006. Intracellular versus cell surface assembly of retroviral pseudotypes is determined by the cellular localization of the viral glycoprotein, its capacity to interact with Gag, and the expression of the Nef protein. J Biol Chem 281:528-42.

127. Pinter A, Honnen WJ, Tung JS, O'Donnell PV, Hämmerling U. 1982. Structural domains of endogenous murine leukemia virus gp 70s containing specific antigenic determinants defined by monoclonal antibodies. Virology 116:499-516.

128. Dodev TS, Karagiannis P, Gilbert AE, Josephs DH, Bowen H, James LK, Bax HJ, Beavil R, Pang MO, Gould HJ, Karagiannis SN, Beavil AJ. 2014. A tool kit for rapid cloning and expression of recombinant antibodies. Sci Rep 4:5885.

129. Janaka SK, Gregory DA, Johnson MC. 2013. Retrovirus glycoprotein functionality requires proper alignment of the ectodomain and the membrane-proximal cytoplasmic tail. J Virol 87:12805-13.

130. Salamango DJ, Johnson MC. 2015. Characterizing the Murine Leukemia Virus envelope glycoprotein membrane-spanning domain for its roles in interface alignment and fusogenicity. J Virol 89:12492-500.

131. Gregory DA, Olinger GY, Lucas TM, Johnson MC. 2014. Diverse viral glycoproteins as well as CD4 co-package into the same Human Immunodeficiency Virus (HIV-1) particles. Retrovirology 11. 
132. Mazurov D, Ilinskaya A, Heidecker G, Lloyd P, Derse D. 2010. Quantitative comparison of HTLV-1 and HIV-1 cell-to-cell infection with new replication dependent vectors. PLoS Pathog 6:e1000788.

133. Aloia AL, Duffy L, Pak V, Lee KE, Sanchez-Martinez S, Derse D, Heidecker G, Cornetta K, Rein A. 2013. A reporter system for replication-competent gammaretroviruses: the inGluc-MLV-DERSE assay. Gene Ther 20:169-76.

134. Gregory DA, Lyddon TD, Johnson MC. 2012. Multiple Gag domains contribute to selective recruitment of Murine Leukemia Virus (MLV) Env to MLV virions. Journal of Virology 87:1518-1527.

135. Chang LJ, Urlacher V, Iwakuma T, Cui Y, Zucali J. 1999. Efficacy and safety analyses of a recombinant Human Immunodeficiency Virus type 1 derived vector system. Gene Ther 6:715-728.

136. Jorgenson RL, Vogt VM, Johnson MC. 2009. Foreign glycoproteins can be actively recruited to virus assembly sites during pseudotyping. J Virol 83:4060-7.

137. Boussif O, Lezoualc'h F, Zanta MA, Mergny MD, Scherman D, Demeneix B, Behr JP. 1995. A versatile vector for gene and oligonucleotide transfer into cells in culture and in vivo: polyethylenimine. Proc Natl Acad Sci U S A 92:7297-7301.

138. Chesebro B, Wehrly K, Nishio J, Perryman S. 1992. Macrophage-tropic Human Immunodeficiency Virus isolates from different patients exhibit unusual V3 envelope sequence homogeneity in comparison with T-cell-tropic isolates: definition of critical amino acids involved in cell tropism. J Virol 66:6547-6554.

139. Chesebro B, Britt W, Evans L, Wehrly K, Nishio J, Cloyd M. 1983. Characterization of monoclonal antibodies reactive with murine leukemia viruses: 
use in analysis of strains of friend MCF and Friend ecotropic Murine Leukemia Virus. Virology 127:134-148.

140. Freed EO, Martin MA. 1995. Virion incorporation of envelope glycoproteins with long but not short cytoplasmic tails is blocked by specific, single amino acid substitutions in the Human Immunodeficiency Virus type 1 matrix. J Virol 69:1984-1989.

141. Mammano F, Kondo E, Sodroski J, Bukovsky A, Göttlinger HG. 1995. Rescue of Human Immunodeficiency Virus type 1 matrix protein mutants by envelope glycoproteins with short cytoplasmic domains. J Virol 69:3824-30.

142. Freed EO, Martin MA. 1996. Domains of the Human Immunodeficiency Virus type 1 matrix and gp41 cytoplasmic tail required for envelope incorporation into virions. J Virol 70:341-51.

143. Ono A, Huang M, Freed EO. 1997. Characterization of Human Immunodeficiency Virus type 1 matrix revertants: effects on virus assembly, Gag processing, and Env incorporation into virions. J Virol 71:4409-18.

144. Brandano L, Stevenson M. 2012. A highly conserved residue in the C-terminal helix of HIV-1 matrix is required for envelope incorporation into virus particles. J Virol 86:2347-59.

145. Tedbury PR, Ablan SD, Freed EO. 2013. Global rescue of defects in HIV-1 envelope glycoprotein incorporation: implications for matrix structure. PLoS Pathog 9:e1003739.

146. Tedbury PR, Mercredi PY, Gaines CR, Summers MF, Freed EO. 2015. Elucidating the mechanism by which compensatory mutations rescue an HIV-1 matrix mutant 
defective for Gag membrane targeting and envelope glycoprotein incorporation. J Mol Biol 427:1413-1427.

147. Kiernan RE, Freed EO. 1998. Cleavage of the Murine Leukemia Virus transmembrane Env protein by Human Immunodeficiency Virus type 1 protease: transdominant inhibition by matrix mutations. J Virol 72:9621-27.

148. Tedbury PR, Novikova M, Ablan SD, Freed EO. 2016. Biochemical evidence of a role for matrix trimerization in HIV-1 envelope glycoprotein incorporation. Proc Natl Acad Sci U S A 113:E182-90.

149. Yang C, Compans RW. 1996. Analysis of the cell fusion activities of chimeric Simian Immunodeficiency Virus-Murine Leukemia Virus envelope proteins: inhibitory effects of the R peptide. J Virol 70:248-54.

150. Taylor GM, Sanders DA. 2003. Structural criteria for regulation of membrane fusion and virion incorporation by the Murine Leukemia Virus TM cytoplasmic domain. Virology 312:295-305.

151. Yang C, Compans RW. 1997. Analysis of the Murine Leukemia Virus R peptide: delineation of the molecular determinants which are important for its fusion inhibition activity. J Virol 71:8490-6.

152. Epand RF, Zhang YL, Mirzabekov T, Kagan B, Silberstein A, Hubbell WL, Epand RM, Chakraborti S, Dimitrov DS, Anderson WF, Rozenberg-Adler Y. 2008. Membrane activity of an amphiphilic alpha-helical membrane-proximal cytoplasmic domain of the MoMuLV envelope glycoprotein. Exp Mol Pathol 84:917. 
153. Rozenberg-Adler Y, Conner J, Aguilar-Carreno H, Chakraborti S, Dimitrov DS, Anderson WF. 2008. Membrane-proximal cytoplasmic domain of Moloney murine leukemia virus envelope tail facilitates fusion. Exp Mol Pathol 84:18-30.

154. Janaka SK, J. F, Johnson MC. 2013. Functional complementation of a model target to study Vpu sensitivity. PloS One 8 .

155. Lukashev AN, Zamyatnin AA, Jr. 2016. Viral Vectors for Gene Therapy: Current State and Clinical Perspectives. Biochemistry (Mosc) 81:700-8.

156. Vargas JE, Chicaybam L, Stein RT, Tanuri A, Delgado-Canedo A, Bonamino MH. 2016. Retroviral vectors and transposons for stable gene therapy: advances, current challenges and perspectives. J Transl Med 14:288.

157. Cosson P. 1996. Direct interaction between the envelope and matrix proteins of HIV-1. EMBO J 15:5783-5788.

158. Manrique JM, Affranchino JL, Gonzalez SA. 2008. In vitro binding of simian immunodeficiency virus matrix protein to the cytoplasmic domain of the envelope glycoprotein. Virology 374:273-9.

159. Pickl WF, Pimentel-Muinos FX, Seed B. 2001. Lipid rafts and pseudotyping. J Virol 75:7175-83.

160. Briggs JA, Wilk T, Fuller SD. 2003. Do lipid rafts mediate virus assembly and pseudotyping? J Gen Virol 84:757-68.

161. Krementsov DN, Rassam P, Margeat E, Roy NH, Schneider-Schaulies J, Milhiet P, Thali M. 2010. HIV-1 assembly differentially alters dynamics and partitioning of tetraspanins and raft components. Traffic 11:1401-1414.

162. Waheed AA, Freed EO. 2010. The Role of Lipids in Retrovirus Replication. Viruses 2:1146-1180. 
163. Sheehy AM, Gaddis NC, Choi JD, Malim MH. 2002. Isolation of a human gene that inhibits HIV-1 infection and is suppressed by the viral Vif protein. Nature 418:646-650.

164. Swanson CM, Puffer BA, Ahmad KM, Doms RW, Malim MH. 2004. Retroviral mRNA nuclear export elements regulate protein function and virion assembly. EMBO J 23:2632-2640.

165. Kothe DL, Decker JM, Li Y, Weng Z, Bibollet-Ruche F, Zammit KP, Salazar MG, Chen Y, Salazar-Gonzalez JF, Moldoveanu Z, Mestecky J, Gao F, Haynes BF, Shaw GM, Muldoon M, Korber BTM, Hahn BH. 2007. Antigenicity and immunogenicity of HIV-1 consensus subtype B envelope glycoproteins. Virology $360: 218-234$.

166. Sanjana NE, Shalem O, Zhang F. 2014. Improved vectors and genome-wide libraries for CRISPR screening. Nat Methods 11:783-784.

167. Varthakavi V, Browning PJ, Spearman P. 1999. Human immunodeficiency virus replication in a primary effusion lymphoma cell line stimulates lytic-phase replication of Kaposi's sarcoma-associated herpesvirus. J Virol 73:10329-38.

168. Mammano F, Salvatori F, Indraccolo S, De Rossi A, Chieco-Bianchi L, Göttlinger HG. 1997. Truncation of the human immunodeficiency virus type 1 envelope glycoprotein allows efficient pseudotyping of Moloney murine leukemia virus particles and gene transfer into CD4+ cells. J Virol 71:3341-5.

169. Zhang C, Hu B, Xiao L, Liu Y, Wang P. 2014. Pseudotyping lentiviral vectors with lymphocytic choriomeningitis virus glycoproteins for transduction of dendritic cells and in vivo immunization. Hum Gene Ther Methods 25:328-38. 
170. Fe Medina M, Kobinger GP, Rux J, Gasmi M, Looney DJ, Bates P, Wilson JM. 2003. Lentiviral vectors pseudotyped with minimal filovirus envelopes increased gene transfer in murine lung. Molecular Therapy 8:777-789.

171. Watanabe M, Hatakeyama S. 2017. TRIM proteins and diseases. J Biochem $161: 135-144$

172. Grutter MG, Luban J. 2012. TRIM5 structure, HIV-1 capsid recognition, and innate immune signaling. Curr Opin Virol 2:142-50.

173. Uchil PD, Quinlan BD, Chan W, Luna JM, Mothes W. 2008. TRIM E3 ligases interfere with early and late steps of the retroviral life cycle. PLoS Pathog 4.

174. Uchil PD, Hinz A, Siegel S, Coenen-Stass A, Pertel T, Luban J, Mothes W. 2013. TRIM protein-mediated regulation of inflammatory and innate immune signaling and its association with antiretroviral activity. J Virol 87:257-72.

175. Takata MA, Goncalves-Carneiro D, Zang TM, Soll SJ, York A, Blanco-Melo D, Bieniasz PD. 2017. CG dinucleotide suppression enables antiviral defence targeting non-self RNA. Nature 550:124-127.

176. Zheng X, Wang X, Tu F, Wang Q, Fan Z, Gao G. 2017. TRIM25 is required for the antiviral activity of zinc finger antiviral protein. J Virol 91.

177. Li MM, Lau Z, Cheung P, Aguilar EG, Schneider WM, Bozzacco L, Molina H, Buehler E, Takaoka A, Rice CM, Felsenfeld DP, MacDonald MR. 2017. TRIM25 Enhances the Antiviral Action of Zinc-Finger Antiviral Protein (ZAP). PLoS Pathog 13:e1006145.

178. Göttlinger HG, Dorfman T, Cohen EA, Haseltine WA. 1993. Vpu protein of human immunodeficiency virus type 1 enhances the release of capsids produced by gag 
gene constructs of widely divergent retroviruses. Proc Natl Acad Sci U S A 90:7381-5.

179. Jin L, Guzik BW, Bor YC, Rekosh D, Hammarskjöld ML. 2002. Tap and NXT promote translation of unspliced mRNA. Genes Dev 17:3075-3086.

180. Bartels H, Luban J. 2014. Gammaretroviral pol sequences act in cis to direct polysome loading and NXF1/NXT-dependent protein production by gag-encoded RNA. Retrovirology 11:73.

181. Miller AD. 2014. Retroviral vectors: From cancer viruses to therapeutic tools. Hum Gene Ther 25:989-995.

182. Nalindi L, Trono D, Verma IM. 2016. Lentiviral vectors, two decades later. Science $353: 1101-1102$.

183. Dunbar CE, High KA, Joung JK, Kohn DB, Ozawa K, Sadelain M. 2018. Gene therapy comes of age. Science 359 .

184. Arias JF, Heyer LN, von Bredow B, Weisgrau KL, Moldt B, Burton DR, Rakasz EG, Evans DT. 2014. Tetherin antagonism by Vpu protects HIV-infected cells from antibody-dependent cell-mediated cytotoxicity. Proc Natl Acad Sci U S A 111:6425-30.

185. Pham TN, Lukhele S, Hajjar F, Routy JP, Cohen EA. 2014. HIV Nef and Vpu protect HIV-infected CD4+ T cells from antibody-mediated cell lysis through down-modulation of CD4 and BST2. Retrovirology 11. 


\section{VITA}

Yul Eum Song was born in Seoul, South Korea in July 1985. She liked reading and had a dream to become an author during the childhood. She joined the philosophy department in Dongguk University, and had a new dream, to become useful to make contributions to the world. She became interested in studying biology, joined a virology lab to work on Kaposi's sarcoma-associated herpesvirus (KSHV), and graduated with two majors, philosophy and life science. Under the influence of her mother, Dr. Mi Hyung Kim, an environmental engineer, she decided to pursue a Ph.D. in virology. She came to the University of Missouri, USA, to study the mechanism of HIV at the frontier of the research field. During 2012-2019, she worked on retroviruses under the guidance of Dr. Marc Johnson. She moves to the next step as a postdoc in St Jude Research Hospital to get closer to her dream. She also writes stories for a hobby in her free time, hoping to have her both dreams come true in the future. 